TRANSACTIONS OF THE

AMERICAN MATHEMATICAL SOCIETY

Volume 361, Number 6, June 2009, Pages 2959-3000

S 0002-9947(08)04496-6

Article electronically published on December 23, 2008

\title{
CERTAIN OPTIMAL CORRESPONDENCES BETWEEN PLANE CURVES, I: MANIFOLDS OF SHAPES AND BIMORPHISMS
}

\author{
DAVID GROISSER
}

\begin{abstract}
In previous joint work, a theory introduced earlier by Tagare was developed for establishing certain kinds of correspondences, termed bimorphisms, between simple closed regular plane curves of differentiability class at least $C^{2}$. A class of objective functionals was introduced on the space of bimorphisms between two fixed curves $C_{1}$ and $C_{2}$, and it was proposed that one define a "best non-rigid match" between $C_{1}$ and $C_{2}$ by minimizing such a functional. In this paper we prove several theorems concerning the nature of the shape-space of plane curves and of spaces of bimorphisms as infinite-dimensional manifolds. In particular, for $2 \leq j<\infty$, the space of parametrized bimorphisms is a differentiable Banach manifold, but the space of unparametrized bimorphisms is not. Only for $C^{\infty}$ curves is the space of bimorphisms an infinite-dimensional manifold, and then only a Fréchet manifold, not a Banach manifold. This paper lays the groundwork for a companion paper in which we use the Nash Inverse Function Theorem and our results on $C^{\infty}$ curves and bimorphisms to show that if $\Gamma$ is strongly convex, if $C_{1}$ and $C_{2}$ are $C^{\infty}$ curves whose shapes are not too dissimilar $\left(C^{j}\right.$-close for a certain finite $j$ ) and if neither curve is a perfect circle, then the minimum of a regularized objective functional exists and is locally unique.
\end{abstract}

\section{INTRODUCTION}

In many problems in science, medicine, and technology the problem of "patternmatching" arises: finding optimal correspondence, in some reasonable sense, between two plane images. One important case is the matching of two plane curves, and there is now a large body of literature on this subject (for a survey of methods and an extensive bibliography through 1995 see [Lo; more recent papers include CAS, FB, SKK, T, TOG]). In this paper and its sequel [G1 we confine ourselves to the problem of matching two simple closed curves in the plane, although much of the work in these papers can be adapted to the problem of matching non-closed or non-simple curves.

In TOG] a theory initially introduced in T] was developed for certain kinds of correspondences, termed bimorphisms, between simple closed regular plane curves of differentiability class at least $C^{2}$. A bimorphism between two such plane curves $C_{1}, C_{2}$ is a certain type of curve $\Sigma$ in the torus $C_{1} \times C_{2}$; for a precise definition see \$2 Given a "cost function" $\Gamma: \mathbf{R} \rightarrow \mathbf{R}-$ a continuous, even function achieving a

Received by the editors April 5, 2004 and, in revised form, February 11, 2007.

2000 Mathematics Subject Classification. Primary 53A04, 49K15.

Key words and phrases. Shape analysis, shape space, non-rigid correspondence, plane curve, bimorphism. 
minimum value of 0 uniquely at 0 , with $\Gamma(|x|)$ increasing in $|x|$ - one can define an objective functional on the set of bimorphisms by

$$
J_{\left(C_{1}, C_{2}\right)}(\Sigma)=\int_{\Sigma} \Gamma\left(\left|\frac{d \mu_{1}}{d s_{\Sigma}}\right| \kappa_{1}\left(\mu_{1}\left(s_{\Sigma}\right)\right)-\left|\frac{d \mu_{2}}{d s_{\Sigma}}\right| \kappa_{2}\left(\mu_{2}\left(s_{\Sigma}\right)\right)\right) d s_{\Sigma} .
$$

Here $s_{\Sigma}$ is an arclength parameter along $\Sigma$ (with respect to a Riemannian metric on $C_{1} \times C_{2}$ in which each factor has length 1$), s_{\Sigma} \mapsto\left(\mu_{1}\left(s_{\Sigma}\right), \mu_{2}\left(s_{\Sigma}\right)\right) \in C_{1} \times$ $C_{2}$ is the corresponding parametrization of $\Sigma$, the vertical bars denote norm of a vector in $\mathbf{R}^{2}$, and $\kappa_{i}$ is the curvature of $C_{i}$. Geometrically, the argument of $\Gamma$ - the "pointwise mismatch" - is the difference in the rates of change, with respect to the common parameter $s_{\Sigma}$, of the direction of the unit tangent vectors to $C_{1}$ and $C_{2}$. A bimorphism that minimizes $J_{\left(C_{1}, C_{2}\right)}$ is deemed to be a "best match" between $C_{1}$ and $C_{2}$. Some advantages of this framework are discussed in T, [TOG], and [G1.

For general curves $C_{1}, C_{2}$ it is not known, for any $\Gamma$, whether a minimizer of $J_{\left(C_{1}, C_{2}\right)}$ exists. However, in most approaches to curve-matching problems, it is not the curves themselves that are directly matched. Rather, one parametrizes each curve by arclength over intervals $\left[0, \ell_{i}\right]$, where $\ell_{i}$ is the arclength of $C_{i}$, and looks for an optimal correspondence between $\left[0, \ell_{1}\right]$ and $\left[0, \ell_{2}\right]$ (cf. [T]). This turns the problem into a search within the space of correspondences between $\left[0, \ell_{1}\right]$ and $\left[0, \ell_{2}\right]$, a space that (except for the numbers $\ell_{1}$ and $\ell_{2}$ ) does not see the curves $C_{1}, C_{2}$ at all. Since the functional (1.1) is insensitive to the sizes of $C_{1}$ and $C_{2}$, we can always rescale $C_{1}$ and $C_{2}$ to have arclength 1 , so that our search space is a fixed space of "internal bimorphisms", a certain set of correspondences between $[0,1]$ and $[0,1]$. Since we are restricting attention to closed curves, our correspondences must satisfy periodic boundary conditions; thus our space of internal bimorphisms $\mathcal{B}^{\text {int }}$ is really a set of correspondences between two fixed circles $S^{1}$ of circumference 1 , each arising as $\mathbf{R} / \mathbf{Z}$. This search space $\mathcal{B}^{\text {int }}$ is completely independent of the curves $C_{1}$ and $C_{2}$.

This suggests an alternative viewpoint on minimizing (1.1). Instead of viewing $C_{1}$ and $C_{2}$ as fixed curves, we can view their curvature functions $\kappa_{1}, \kappa_{2}$ as parametrizing a family of objective functionals $\Sigma \mapsto J\left(\Sigma ; \kappa_{1}, \kappa_{2}\right)$ on the space $\mathcal{B}^{\text {int }}$. The advantage of this approach is that it allows use of the

Stability Principle. Stable minima persist in suitably differentiable families of suitably differentiable functions.

Thus if one knows that one member of a parametrized family of functionals has a stable minimum, one may be able to conclude that every nearby functional will have a stable minimum nearby. In our situation, the functional $J$ trivially achieves some absolute minima: if $C_{1}=C_{2}$ then $J_{\left(C_{1}, C_{2}\right)}$ achieves its absolute minimum of zero at the bimorphism corresponding to the identity map $C_{1} \rightarrow C_{2}$. In terms of the parametrized family of functionals on $\mathcal{B}^{\text {int }}$, this says that for all curvature functions $\kappa_{0}$, the functional $J\left(\cdot ; \kappa_{0}, \kappa_{0}\right)$ achieves its absolute minimum, namely zero, at the internal bimorphism $\Delta$ corresponding to the identity map $S^{1} \rightarrow S^{1}$.

Using this idea we prove in G1 an existence/uniqueness theorem, a weaker version of which is stated below, for minima of a slightly perturbed (regularized) version of $J$. Our perturbation replaces $J$ by $J^{\varepsilon}:=J+\varepsilon Q$, where $\varepsilon>0$ and $Q$ is a certain functional on $\mathcal{B}^{\text {int }}$ that penalizes deviation from "uniformity", a perfectly uniform internal bimorphism $\Sigma^{\text {int }}$ being one in which, under a parametrization, the two factors of $S^{1} \times S^{1}$ are traversed at identically equal rates. (See [G1] for the 
precise definition of Q.) Our proof of this theorem involves an implicit-function theorem and therefore requires us to know that a suitable open subset of $\mathcal{B}^{\text {int }}$ is a manifold (infinite-dimensional of course), and that so is the parameter-space $\widetilde{\mathcal{S}}$ in which the normalized curvature functions $\kappa_{i}$ (see $₫ 2$ live. This paper is devoted to the structure of these spaces.

To state the existence/uniqueness theorem we require some terminology that will be elaborated upon in $\$ 2$. By a based plane curve we will mean a pair $(C, q)$, where $C$ is a simple closed curve in $\mathbf{R}^{2}$ and $q \in C$, and by a based shape we will mean the equivalence class of a based curve under basepoint-preserving positivelyoriented similarity transformations. For $0 \leq j \leq \infty$ we define the shape space $\mathcal{S}_{j}$ (respectively, based-shape space $\widetilde{\mathcal{S}}_{j}$ ) of curves in the plane to be the sets of shapes (resp. based shapes) of $C^{j}$ plane curves. Given two based plane curves $C_{i, b}=\left(C_{i}, q_{i}\right), i=1,2$, the pseudo-identity $\left(C_{1}, C_{2}\right)$-bimorphism $\Sigma_{\mathrm{id}}\left(C_{1, b}, C_{2, b}\right) \subset$ $C_{1} \times C_{2}$ is the graph of the map $C_{1} \rightarrow C_{2}$ determined by linearly interpolating between the arclength function of $C_{1}$ based at $q_{1}$ and the arclength function of $C_{2}$ based at $q_{2}$. We denote by $\mathcal{B}_{j,+}\left(C_{1}, C_{2}\right)$ the space of orientation-preserving $C^{j}$ bimorphisms between $C_{1}$ and $C_{2}$. The perturbed functional $J^{\varepsilon}$ determines a functional $J_{\left(C_{1, b}, C_{2, b}\right)}^{\varepsilon}: \mathcal{B}_{j,+}\left(C_{1}, C_{2}\right) \rightarrow \mathbf{R}$.

Theorem 1.1. Fix $\varepsilon>0$. Let $C_{0, b}$ be a based, $C^{\infty}$, simple closed curve in $\mathbf{R}^{2}$ with based shape $\left[C_{0, b}\right] \in \widetilde{\mathcal{S}}_{\infty}$. Assume that $C_{0, b}$ is not a perfect circle. Then there exist $j_{1}, j_{2}<\infty$ and an open $C^{j_{1}}$-neighborhood $U$ of $\left[C_{0, b}\right]$ in $\widetilde{\mathcal{S}}_{\infty}$ such that for all based curves $C_{1, b}, C_{2, b}$ with $\left[C_{i, b}\right] \in U$, there exist an open $C^{j_{2}}$-neighborhood $W$ of $\Sigma_{\mathrm{id}}\left(C_{1, b}, C_{2, b}\right)$ in $\mathcal{B}_{\infty,+}\left(C_{1}, C_{2}\right)$ and a unique $\left(C_{1}, C_{2}\right)$-bimorphism $\Sigma \in W$ at which the restriction of $J_{\left(C_{1, b}, C_{2, b}\right)}^{\varepsilon}$ to $W$ is minimized.

A useful corollary of Theorem 1.1 is the special case in which one of the based curves $C_{1, b}, C_{2, b}$ is $C_{0, b}$ itself. It may also be possible to use Theorem 1.1 to prove results for $C^{j}$ curves, $j<\infty$, by taking limits of minimizers for $C^{\infty}$ curves.

The reason that only $C^{\infty}$ curves appear in Theorem 1.1 goes back to the Stability Principle, which we stated with deliberate vagueness because its hypotheses depend on context. In all contexts, a "stable minimum" is (at least) an isolated local minimum; in some contexts there are more restrictions. For finite-dimensional families of functions defined on finite-dimensional manifolds $M$ (or on compact domains in $\mathbf{R}^{n}$, with appropriate boundary conditions), "suitably differentiable" in the Stability Principle means $C^{2}$, and "stable minimum" of a function $F$ can be replaced by "infinitesimally stable minimum", one at which the Hessian of $F$ is strictly positive-definite. In this context the Stability Principle is proven applying the standard Implicit Function Theorem to the gradient of $F$ (more precisely, to the parametrized family of these gradients) with respect to an arbitrary Riemannian metric. The proof relies on the fact that in finite dimensions every non-degenerate critical point $x_{0}$ is strongly non-degenerate: the metric and the non-degenerate Hessian combine to give an isomorphism from the tangent space $T_{x_{0}} M$ to itself.

The space of internal bimorphisms is of course infinite-dimensional, as is the parameter-space of pairs of normalized curvature functions $\left(\kappa_{1}, \kappa_{2}\right)$. The BanachSpace Implicit Function Theorem leads to a form of the Stability Principle for infinite-dimensional $C^{2}$ Banach manifolds. However, strict positivity of the Hessian $H$ of $F$ at $x_{0}$ is no longer enough, not even guaranteeing that $F$ has local minimum at $x_{0}$; one needs strong positivity: $H(v, v) \geq c_{1}\|v\|^{2}$ for some $c_{1}>0$. 
It would seem a reasonable strategy to try to prove a version of Theorem 1.1 for finitely-differentiable curves by attempting to endow $\mathcal{B}_{j}^{\text {int }}$, the space of internal bimorphisms of class $C^{j}$, with the structure of a Banach manifold for some appropriate finite $j$, and attempting to apply the Stability Principle. The problem with this approach is that in our situation essentially none of the hypotheses of the Banach-Space Implicit Function Theorem are satisfied: the domain $\mathcal{M}$ of the relevant map $Z\left(\cdot ; \kappa_{1}, \kappa_{2}\right)$ (the $L^{2}$-gradient of $J$ with $\kappa_{1}, \kappa_{2}$ held fixed) is not a Banach manifold; at most points of $\mathcal{M}$ the map $Z$ is not differentiable in the Banach-space sense; and at the points at which $Z$ has an invertible derivative with respect to its first argument, the inverse of the derivative is not bounded.

The last problem is the easiest to surmount; it is solved by adding the regularization term to $J$. To understand the other problems it is important to distinguish between parametrized $C^{j}$ bimorphisms, the space of which we denote $\widetilde{\mathcal{B}}_{j}^{\text {int }}$, and unparametrized bimorphisms. From the definitions in $₫ 2$ it will be clear that $\widetilde{\mathcal{B}}_{j}^{\text {int }}$ has a boundary, hence cannot be a manifold, but this is a trivial difficulty easily solved by enlarging $\widetilde{\mathcal{B}}_{j}^{\text {int }}$ to a space $\widetilde{\mathcal{M}}$ int of parametrized $C^{j}$ curves in $S^{1} \times S^{1}$ that we call parametrized h-bimorphisms because they are homotopic to parametrized bimorphisms; similarly we enlarge $\mathcal{B}_{j}^{\text {int }}$ to a space $\mathcal{M}_{j}^{\text {int }}$. The space $\widetilde{\mathcal{M}}_{j}^{\text {int }}$ is a Banach manifold for every finite $j \geq 1$, and the lifted functionals $\tilde{J}$ and $\tilde{J}^{\varepsilon}$ extend to $C^{j}$ functionals on $\widetilde{\mathcal{M}}_{j}^{\text {int }} \times \widetilde{\mathcal{S}}_{j+2} \times \widetilde{\mathcal{S}}_{j+2}$. However, at every point of $\widetilde{\mathcal{M}}_{j}^{\text {int }}$ the Hessian of $\tilde{J}_{\left(C_{1}, C_{2}\right)}$ (and $\left.\tilde{J}_{\left(C_{1}, C_{2}\right)}^{\varepsilon}\right)$ has a nontrivial nullspace, owing to the parametrizationindependence of the functional. To get a Hessian that has a chance to be positivedefinite, one must pass to the quotient $\mathcal{M}_{j}^{\text {int }}$. However, for no $j$ is $\mathcal{M}_{j}^{\text {int }}$ a differentiable Banach manifold. For finite $j$ it is only a $C^{0}$-manifold, and one cannot even talk of a $C^{1}$ function, let alone apply an implicit-function theorem. One can make sense of the Banach-space derivative of $J$ on the subset $\mathcal{M}_{j+1}^{\text {int }} \subset \mathcal{M}_{j}^{\text {int }}$, but this is too small a subset to be of any use.

This forces us to consider $C^{\infty}$ curves. The spaces $\widetilde{\mathcal{M}}_{\infty}^{\text {int }}$ and $\mathcal{M}_{\infty}^{\text {int }}$ are not Banach manifolds, but tame Fréchet manifolds (see the Appendix for this terminology), so the Banach-space Implicit Function Theorem and its associated stability principle are not available to us. What is available is the Implicit Function Theorem associated with the Nash-Moser Inverse Function Theorem for Fréchet spaces (see the Appendix). From this we can derive a stability principle tailored to our situation, allowing us to prove Theorem 1.1. Unfortunately, the hypotheses of the NashMoser theorem are enormously harder to verify than are those of the Banach-space theorem. Consequently, the proofs in this paper and [G1 are quite long. They may appear superficially to be a matter of crank-turning, but this appearance is deceptive: in the middle of a proof one finds that one has to invent the right crank to turn.

The first major purpose of this paper is to lay the groundwork for the proof of Theorem 1.1 by establishing what types of manifolds various spaces of shapes and bimorphisms are (Theorems 2.8 2.9 and Corollary 2.10). The second major purpose is to facilitate future work in this area, and for this purpose Theorems 2.82 .9 include statements concerning the spaces $\widetilde{\mathcal{M}}_{j}^{\text {int }}, \mathcal{M}_{j}^{\text {int }}$, and $\widetilde{\mathcal{S}}_{j}$ for finite as well as infinite $j$, although only the infinite- $j$ spaces are of consequence to Theorem 1.1. We also prove that $\widetilde{\mathcal{S}}_{\infty}$ is a tame Fréchet manifold even though this too is not needed for the proof of Theorem 1.1. 
Among the spaces we study are the spaces of constant-speed $C^{j}$ internal hbimorphisms $\widetilde{\mathcal{M}}_{j}^{\text {int }, c}$. We prove that these are $C^{\infty}$ Banach manifolds for $2 \leq j<\infty$ and a tame Fréchet manifold for $j=\infty$ (the proof of the latter being surprisingly laborious). $\widetilde{\mathcal{M}}_{j}^{\text {int,c }}$ is a circle-bundle over $\mathcal{M}_{j}^{\text {int }}$, and we use this relation to give us the manifold structure on $\mathcal{M}_{j}^{\text {int }}$. It is possible to prove Theorem 1.1 without using $\widetilde{\mathcal{M}}_{\infty}^{\text {int, },}$, but it seems worthwhile for several reasons to understand and use $\widetilde{\mathcal{M}}_{\infty}^{\text {int,c }}$ (and $\widetilde{\mathcal{M}}_{j}^{\text {int,c }}$ for $j<\infty$ ). First, in most applications practitioners prefer to use "standard" (usually unit-speed) parametrizations. There is a natural one-to-one correspondence between constant-speed parametrized (h)-bimorphisms and unitspeed parametrized (h)-bimorphisms, so our results concerning $\widetilde{\mathcal{M}}_{\infty}^{\text {int,c }}$ (Corollary 2.10) imply that the space of unit-speed h-bimorphisms is a tame Fréchet manifold as well. Second, various formulas related to the first and second derivatives of $\tilde{J}_{\left(C_{1}, C_{2}\right)}^{\varepsilon}$ (computed in G1]) would be more complicated if we used $\widetilde{\mathcal{M}}_{\infty}^{\text {int }}$ rather than $\widetilde{\mathcal{M}}_{\infty}^{\text {int,c }}$ to give us the manifold structure on $\mathcal{M}_{\infty}^{\text {int }}$. Third, obtaining $\mathcal{M}_{\infty}^{\text {int }}$ from $\widetilde{\mathcal{M}}_{\infty}^{\text {int }}$ involves modding out by the diffeomorphism group of the circle, which presents its own problems. Finally, obtaining $\mathcal{M}_{j}^{\text {int }}$ as $\widetilde{\mathcal{M}}_{j}^{\text {int,c }} / S^{1}$ rather than as $\widetilde{\mathcal{M}}_{j}^{\text {int }} / \operatorname{Diff}_{j}^{+}\left(S^{1}\right)$ makes it clear that for finite $j$, the fact that $\mathcal{M}_{j}^{\text {int }}$ is no more than a $C^{0}$ manifold has nothing to do with the complicated nature of the diffeomorphism group; all by itself the circle-action on $\widetilde{\mathcal{M}}_{j}^{\text {int,c }}$-rotations of the circle used to parametrize (h-)bimorphisms - is badly enough behaved to rob $\mathcal{M}_{j}^{\text {int }}$ of any chance of being a differentiable object. This fact may be well-known to differential geometers, but not to researchers in image-analysis, for whom we also wish this paper to be informative and accessible.

This paper is organized as follows. In $₫ 2$ we carefully define the various types of bimorphisms and their parameter spaces. We also show that shapes of $C^{2}$ simple closed plane curves are in one-to-one correspondence with normalized curvature functions, defined therein. Theorems 2.8 and 2.9 state what type of manifold each parameter-space is. The proofs of these theorems, being long and technical, are deferred to 4 . Readers desiring more detail than we give in these proofs may obtain the preprint G2] from the author.

In the Appendix we also summarize some background material on Fréchet manifolds and the Nash-Moser Theorem, since this material may not be widely known to researchers in image-analysis. There is already an excellent reference for this material, $[\mathrm{H}$, which we cite liberally; however it did not seem fair simply to refer the reader to this 158-page paper, whose table of contents carries no page numbers, and whose pages give no clue as to whether one is in Chapter I, II, or III. For this reason our citations of $[\mathrm{H}]$ are very detailed.

We conclude this introduction with some notation and terminology. For finitedimensional vector spaces or manifolds $M, N$ and $0 \leq j \leq \infty$ we write $C^{j}(M, N)$ for the space of $C^{j}$ maps from $M$ to $N$. As is customary $C^{j}(M)$ means $C^{j}(M, \mathbf{R})$ and "smooth" means $C^{\infty}$. In this paper, $S^{1}$ denotes the specific model of the circle as $\mathbf{R} / \mathbf{Z}$, with basepoint we denote 0 (the image of $0 \in \mathbf{R}$ under the quotient map). As a quotient of $\mathbf{R}$, this circle inherits the standard Riemannian metric which we use implicitly throughout; thus the circumference of our $S^{1}$ is 1 , not $2 \pi$. We endow $S^{1} \times S^{1}$ with the product metric; $S^{1} \times S^{1}$ will always mean this specific model of the flat torus. The standard variable $t \in \mathbf{R}$ is an arclength parameter for $S^{1}$, which 
we use to canonically identify $\dot{f}$, the derivative with respect to arclength of a map $f: S^{1} \rightarrow \mathbf{R}$ or $f: S^{1} \rightarrow S^{1}$, as a map $S^{1} \rightarrow \mathbf{R}$. For the standard inner product on $\mathbf{R}^{2}$ and the induced Riemannian metrics on $\mathbf{R}^{2}$ and $S^{1} \times S^{1}$ we generally use dot-product notation, except for some long formulas in which parenthesis-notation $(\cdot, \cdot)$ is more convenient. Norms of vectors in $\mathbf{R}^{2}$ are denoted with single bars $|\cdot|$; we reserve double-bars for function-space norms.

For $1 \leq j \leq \infty$, a $C^{j}$ parametrized regular closed curve in the plane is a $C^{j}$ embedding $S^{1} \rightarrow \mathbf{R}^{2}$, i.e. a $C^{j}$ one-to-one map $t \mapsto(x(t), y(t))$ with $\dot{x}(t), \dot{y}(t)$ never vanishing simultaneously. A $C^{j}$ regular closed curve in the plane is the image of such a map. The same terminology applies to curves in a torus.

Unless otherwise specified, "(parametrized) curve" means "simple, closed, oriented, (parametrized) regular curve" (either in the plane or a torus) of whatever differentiability class is specified $\left(C^{j}, 1 \leq j \leq \infty\right)$. If no differentiability class is specified, $j \geq 2$ is always assumed (except where explicitly stated otherwise), so that the curvature function of an oriented plane curve is always defined and continuous.

\section{Shapes, BIMORPhisms, AND THEIR PARAMETER SPACES}

In the Introduction we defined the spaces $\mathcal{S}_{j}$ (respectively $\widetilde{\mathcal{S}}_{j}$ ) of shapes (resp. based shapes) of $C^{j}$ curves in $\mathbf{R}^{2}, 0 \leq j \leq \infty$. For $j \geq 1$ there is a one-to-one correspondence between based shapes and based curves $(C, q)$ in the $x y$ plane that satisfy the following "normalization" conditions:

$$
\left\{\begin{array}{l}
\text { 1. } q=(0,0), \\
\text { 2. the positively-oriented tangent direction to } C \text { at } q \\
\text { is the positive } x \text {-direction, } \\
\text { 3. the total arclength of } C \text { is } 1 .
\end{array}\right.
$$

A based plane curve satisfying (2.1) will be called normalized. Since the basepoint of every normalized curve is the origin, we can unambiguously refer to a "normalized curve $C$ ". Because of the natural one-to-one correspondence between based shapes and normalized curves we will often regard $\widetilde{\mathcal{S}}_{j}$ as the space of normalized $C^{j}$ curves. The circle acts on $\widetilde{\mathcal{S}}_{j}$ (as we will see shortly) and on many other spaces in this paper, so we introduce the following

Notation 2.1. For any set $X$, each function $f \in \operatorname{Maps}\left(S^{1}, X\right)$, and each $a \in S^{1}$, define $\tau_{a}(f) \in \operatorname{Maps}\left(S^{1}, X\right)$ by

$$
\left(\tau_{a}(f)\right)(t)=f(t+a) .
$$

Every normalized curve $C$ has a unique positively-oriented unit-speed parametrization $\gamma_{C}: S^{1} \rightarrow C$ with $\gamma(0)=(0,0)$; we call $\gamma_{C}$ the canonical parametrization of $C$. For $1 \leq j \leq \infty$, a curve is $C^{j}$ if and only if its canonical parametrization is $C^{j}$. For any unit-speed parametrization $\gamma: S^{1} \rightarrow C$ and any $a \in S^{1}, \tau_{a}(\gamma)$ is again a unit-speed parametrization of $C$. Thus $\tau$ defines a free, transitive action of $S^{1}$ on the set of unit-speed positively oriented parametrizations of $C$, a set that is in natural one-to-one correspondence with $C$ itself (a choice of basepoint uniquely determines such a parametrization, and vice-versa). Hence there is a natural identification $\mathcal{S}_{j} \cong \widetilde{\mathcal{S}}_{j} / S^{1}$. 
A correspondence between two plane curves $C_{1}, C_{2}$ is a subset $\Sigma$ of the product space $C_{1} \times C_{2}$ such that the projection of $\Sigma$ onto the $i^{\text {th }}$ factor is $C_{i}$. For $p \in C_{1}$, we refer to the set $\left\{q \in C_{2} \mid(p, q) \in \Sigma\right\}$ as the image of $p$ in $C_{2}$ under $\Sigma$, and say that $\Sigma$ matches $p$ with its image in $C_{2}$. We use analogous terminology with the roles of $C_{1}$ and $C_{2}$ reversed.

Any such curve-correspondence can be obtained by applying similarity transformations to a correspondence of normalized curves. The primary interest in curvecorrespondences is thus really an interest in shape-correspondences; a similarity transformation carrying one plane curve to another can be viewed as a "perfect match".

In T] a type of curve-correspondence called a bimorphism was introduced (studied further in [TOG]). Let $C_{1}, C_{2}$ be $C^{l}$ curves in the plane, and let $j \leq l$. A $C^{j}$ bimorphism between $C_{1}$ and $C_{2}$, or $C^{j}\left(C_{1}, C_{2}\right)$-bimorphism, is a $C^{j}$ curve $\Sigma$ in the product $C_{1} \times C_{2}$ that projects onto $C_{1}$ and $C_{2}$ and for which the image under $\Sigma$ of every point in each curve is either a point or an arc in the other curve. (The object called a bimorphism in TOG] is what we are here calling a $C^{1}$ bimorphism.) We call $\Sigma$ an identity bimorphism if $C_{1}=C_{2}$ and $\Sigma=\left\{(q, q) \mid q \in C_{1}\right\}$.

By a normalized $C^{j}$ parametrization of a $\left(C_{1}, C_{2}\right)$-bimorphism $\Sigma$ we will mean a $C^{j}$ embedding $\mu: S^{1} \rightarrow C_{1} \times C_{2}$ with image $\Sigma$. Here "normalized" refers to the fact that $S^{1}$ means the specific model of the circle as $\mathbf{R} / \mathbf{Z}$. "Parametrized bimorphism" will always mean "normalized parametrization of a bimorphism".

Throughout this paper, $t$ is used for the domain variable of a parametrized $\left(C_{1}, C_{2}\right)$-bimorphism, and "dot" denotes $d / d t$. It is shown in TOG] that for $i=1,2$, if $u_{i}$ is a positive-speed parameter along $C_{i}$ and $\pi_{C_{i}}: C_{1} \times C_{2} \rightarrow C_{i}$ is the natural projection, then $u_{i} \circ \pi_{C_{i}} \circ \mu$ is monotone, and hence the "embedding" requirement above is equivalent to the condition that the tangent vector $\dot{\mu}(t)$ is nowhere zero. Thus, given a normalized parametrization $\mu$ of a $\left(C_{1}, C_{2}\right)$-bimorphism, for the pair of maps $\mu_{i}:=\pi_{C_{i}} \circ \mu: S^{1} \rightarrow C_{i}$ the derivatives $\dot{\mu}_{1}(t), \dot{\mu}_{2}(t)$ never vanish simultaneously. We call a $\left(C_{1}, C_{2}\right)$-bimorphism $\Sigma$ orientation-preserving if for a normalized parametrization $\mu$ the maps $\mu_{1}, \mu_{2}$ are both orientation-preserving or both orientation-reversing (a condition independent of the choice of $\mu$ ); otherwise we call $\Sigma$ orientation-reversing.

For $1 \leq j \leq \infty$ let $\mathcal{B}_{j}\left(C_{1}, C_{2}\right)$ (respectively $\widetilde{\mathcal{B}}_{j}\left(C_{1}, C_{2}\right)$ ) denote the set of all $C^{j}$ $\left(C_{1}, C_{2}\right)$-bimorphisms (respectively, parametrized bimorphisms). Thus $\mathcal{B}_{j}\left(C_{1}, C_{2}\right)$ $=\mathcal{B}_{j,+}\left(C_{1}, C_{2}\right) \amalg \mathcal{B}_{j,-}\left(C_{1}, C_{2}\right)$, where the sign indicates whether orientation is preserved; a similar decomposition holds for the parametrized case. Any orientationreversing diffeomorphism of $C_{2}$ induces a bijection $\mathcal{B}_{j,+}\left(C_{1}, C_{2}\right) \leftrightarrow \mathcal{B}_{j,-}\left(C_{1}, C_{2}\right)$. From now on "(parametrized) bimorphism" will always mean "orientation-preserving (parametrized) bimorphism", since all properties of orientation-reversing bimorphisms can be easily recovered from those of orientation-preserving bimorphisms.

Given a $C^{2}$ plane curve $C$, let $\bar{\kappa}_{C}: C \rightarrow \mathbf{R}$ denote its curvature function, with the sign convention that a convex curve has nonnegative curvature if it is oriented counterclockwise. We will restrict our attention to plane curves with the counterclockwise orientation; again it is easy to translate all of what follows below to the other orientation cases.

Definition 2.2. For $0 \leq j \leq \infty$, a normalized $C^{j}$ curvature function is a function $S^{1} \rightarrow \mathbf{R}$ of the form $\kappa_{C}:=\bar{\kappa}_{C} \circ \gamma_{C}$, where $C$ is a normalized based $C^{j+2}$ plane 
curve, oriented counterclockwise, and $\gamma_{C}: S^{1} \rightarrow C$ is the canonical parametrization of $C$.

Not every function on the circle is a normalized curvature function; see Lemma 4.5 .

Let $\widetilde{\mathcal{K}}_{j}$ denote the space of normalized $C^{j}$ curvature functions. Given $a \in S^{1}, \kappa \in$ $\widetilde{\mathcal{K}}_{j}$, the function $\tau_{a}(\kappa): S^{1} \rightarrow \mathbf{R}$ is again a normalized curvature function, arising from the same plane curve but with a different basepoint (if $a \neq 0$ ). Thus the group $S^{1}$ acts on $\widetilde{\mathcal{K}}_{j}$, and we define $\mathcal{K}_{j}=\widetilde{\mathcal{K}}_{j} / S^{1}$.

Normalized curvature functions are useful because they completely classify shapes:

Lemma 2.3. Two based plane curves $C_{1}, C_{2}$ have the same shape if and only if their normalized curvature functions differ by at most a translation: $\kappa_{C_{2}}=\tau_{a}\left(\kappa_{C_{1}}\right)$ for some $a \in S^{1}$. Thus, for $2 \leq j \leq \infty$, there are natural 1-1 correspondences

$$
\begin{aligned}
& \widetilde{\mathcal{K}}_{j-2} \longleftrightarrow \widetilde{\mathcal{S}}_{j}, \\
& \mathcal{K}_{j-2} \longleftrightarrow \mathcal{S}_{j} .
\end{aligned}
$$

Proof. One direction of the implication is trivial. For the other, write $\gamma_{C}(t)=$ $(x(t), y(t))$ and let $u=\dot{x}, v=\dot{y}$. Then $(u, v)$ satisfies the linear ODE $(\dot{u}(t), \dot{v}(t))=$ $\kappa_{C_{1}}(t)(-v(t), u(t))$ with initial conditions $(u(0), v(0))=(1,0)$. If $\kappa_{C_{2}}=\tau_{a}\left(\kappa_{C_{1}}\right)$, let $R$ be the unique positively-oriented rigid motion of $\mathbf{R}^{2}$ carrying $\gamma_{C_{1}}(a)$ to the origin and $\gamma_{C_{1}}^{\prime}(a)$ to $(1,0)$. Since $\kappa_{C_{1}}$ is continuous, it follows from the standard uniqueness theorem for solutions of linear ODE's that $\gamma_{C_{2}}(t)=R\left(\gamma_{C_{1}}(t+a)\right)$ for all $t$, i.e. that $\gamma_{C_{2}}=R\left(\tau_{a}\left(\gamma_{C_{1}}\right)\right)$ and hence that $R$ carries $C_{1}$ to $C_{2}$.

We use the correspondences asserted in Lemma 2.3 to endow $\widetilde{\mathcal{S}}_{j}$ with the differentiable structure on $\widetilde{\mathcal{K}}_{j-2}$ asserted later in Theorem 2.9] and $\mathcal{S}_{j}$ with the corresponding quotient topology.

In view of Lemma2.3, if $C$ is the normalized curve corresponding to the normalized curvature function $\kappa$, we will alternatively use the notation $\gamma_{\kappa}$ for the canonical parametrization $\gamma_{C}$. The proof above shows that

$$
\gamma_{\tau_{a}(\kappa)}=R_{a, \kappa}\left(\tau_{a}\left(\gamma_{\kappa}\right)\right)
$$

for some positively-oriented rigid motion $R_{a, \kappa}$ of $\mathbf{R}^{2}$.

In practical applications, of course, curves do not start out normalized; given a curve $C$ of length $\ell_{C}$ one commonly chooses a basepoint and then uses an arclength parametrization over the interval $\left[0, \ell_{C}\right]$. The curvature of $C$ is then viewed as a function $\bar{\kappa}_{C}:\left[0, \ell_{C}\right] \rightarrow \mathbf{R}$ with periodic boundary conditions, or equivalently a period- $\ell_{C}$ function on $\mathbf{R}$. The corresponding normalized curvature function $\kappa_{C}$ is then given by

$$
\kappa_{C}(t)=\ell_{C} \bar{\kappa}_{C}\left(\ell_{C} t\right) .
$$

Let $p^{\Delta}: S^{1} \rightarrow S^{1} \times S^{1}$ be the diagonal map $t \mapsto(t, t)$, a smooth parametrized curve in the torus. Parametrized curves that are homotopic to $p^{\Delta}$ will play a special role below. We call an oriented curve in the torus (1,1)-homotopic if it admits a positively-oriented parametrization homotopic to $p^{\Delta}$.

Definition 2.4. For $1 \leq j \leq \infty$, let $\widetilde{\mathcal{M}}_{j}^{\text {int }}$ (respectively $\left.\mathcal{M}_{j}^{\text {int }}\right)$ denote the set of $C^{j}$ parametrized curves (resp. oriented curves) in $S^{1} \times S^{1}$ homotopic to $p^{\Delta}$ (resp. (1,1)homotopic), and let $\pi_{\widetilde{\mathcal{M}} \rightarrow \mathcal{M}}: \widetilde{\mathcal{M}}_{j}^{\text {int }} \rightarrow \mathcal{M}_{j}^{\text {int }}$ denote the natural projection. We call 
$\widetilde{\mathcal{M}}_{j}^{\text {int }}$ the space of $C^{j}$ parametrized internal h-bimorphisms (for $\mathcal{M}_{j}^{\text {int }}$ we omit the word "parametrized"), and write a typical element $p \in \widetilde{\mathcal{M}}_{j}^{\text {int }}$ as a pair $\left(p_{1}, p_{2}\right)$, where $p_{i}: S^{1} \rightarrow S^{1}$ is the map obtained by following $p$ with composition onto the $i^{\text {th }}$ factor of $S^{1} \times S^{1}$. We define $\widetilde{\mathcal{B}}_{j}^{\text {int }}$, the space of $C^{j}$ parametrized internal bimorphisms, to be the subset $\left\{\left(p_{1}, p_{2}\right) \in \widetilde{\mathcal{M}}_{j}^{\text {int }} \mid \dot{p}_{i}(t) \geq 0, i=1,2, \forall t \in S^{1}\right\}$, and $\mathcal{B}_{j}^{\text {int }}$, the space of $C^{j}$ internal bimorphisms, to be the image of $\widetilde{\mathcal{B}}_{j}^{\text {int }}$ under $\pi_{\widetilde{\mathcal{M}} \rightarrow \mathcal{M}}$. We let $\widetilde{\mathcal{M}}_{j}^{\text {int }, c} \subset \widetilde{\mathcal{M}}_{j}^{\text {int }}$ denote the subset for which the speed of the parametrization is constant, and $\widetilde{\mathcal{B}}_{j}^{\text {int }, c}:=\widetilde{\mathcal{B}}_{j}^{\text {int }} \cap \widetilde{\mathcal{M}}_{j}^{\text {int }, c}$; we call $\widetilde{\mathcal{M}}_{j}^{\text {int }, c}$ (respectively $\widetilde{\mathcal{B}}_{j}^{\text {int }, c}$ ) the space of $C^{j}$ constantspeed internal h-bimorphisms (respectively bimorphisms.) The parametrized curves $\tau_{a}\left(p^{\Delta}\right), a \in S^{1}$, are called parametrized identity internal bimorphisms; the image of any such curve is the identity internal bimorphism $\Delta$.

For each $p \in \widetilde{\mathcal{M}}_{j}^{\text {int }}$ we denote by $\ell_{p}$ or $\ell_{\Sigma}$ the length of the curve $\Sigma \in \mathcal{M}_{j}^{\text {int }}$ parametrized by $p$. From the unit-square model of the torus, it is easy to see that for all $p \in \widetilde{\mathcal{M}}_{j}^{\text {int }}$ we have $\ell_{p} \geq \sqrt{2}=\ell_{\Delta}$, and for all $p \in \widetilde{\mathcal{B}}_{j}^{\text {int }}$ we have $\sqrt{2} \leq \ell_{p}<2$.

The speed of a constant-speed curve parametrized over $S^{1}$ necessarily equals the length of the curve; our fixed domain $S^{1}$ in the definition of "parametrized curve" prevents us from parametrizing all curves at unit speed. However, there is a natural one-to-one correspondence between the set of constant-speed based curves $C$ parametrized over $S^{1}$ and the set of unit-speed based curves $C^{\prime}$, given by keeping the base point fixed and rescaling the unit-speed parameter so that the rescaled parameter runs over $[0,1]$ instead of $\left[0, \ell_{C^{\prime}}\right]$. Thus every statement in this paper concerning the spaces $\widetilde{\mathcal{M}}_{j}^{\text {int,c }}$ or $\widetilde{\mathcal{B}}_{j}^{\text {int,c }}$ has a translation into a statement about the space of unit-speed curves in $S^{1} \times S^{1}$ homotopic to $p^{\Delta}$.

Let $\operatorname{Diff}_{j}^{+}\left(S^{1}\right)$ denote the group of $C^{j}$ orientation-preserving diffeomorphisms of $S^{1}$; later we write simply " $\operatorname{Diff}^{+}\left(S^{1}\right)$ " for $\operatorname{Diff}_{\infty}^{+}\left(S^{1}\right)$. We have natural one-to-one correspondences

$$
\widetilde{\mathcal{M}}_{j}^{\text {int }} / \operatorname{Diff}_{j}^{+}\left(S^{1}\right) \longleftrightarrow \widetilde{\mathcal{M}}_{j}^{\text {int }, c} / S^{1} \longleftrightarrow \mathcal{M}_{j}^{\text {int }},
$$

and the same with $\mathcal{M}$ replaced by $\mathcal{B}$. We will often use this later to translate problems on $\mathcal{M}_{j}^{\text {int }}$ into $S^{1}$-equivariant (or invariant) problems on $\widetilde{\mathcal{M}}_{j}^{\text {int,c }}$, rather than $\operatorname{Diff}_{j}^{+}\left(S^{1}\right)$-equivariant (or invariant) problems on $\widetilde{\mathcal{M}}_{j}^{\text {int }}$, circumventing certain difficulties associated with the complicated group $\operatorname{Diff}_{j}^{+}\left(S^{1}\right)$.

There is a natural projection $\pi_{\widetilde{\mathcal{M}} \rightarrow \widetilde{\mathcal{M}}^{c}}: \widetilde{\mathcal{M}}_{j}^{\text {int }} \rightarrow \widetilde{\mathcal{M}}_{j}^{\text {int,c }}$ defined by

$$
\pi_{\widetilde{\mathcal{M}} \rightarrow \widetilde{\mathcal{M}}^{c}}(p)=p \circ \sigma_{p}
$$

where $\sigma_{p} \in \operatorname{Diff}_{j}^{+}\left(S^{1}\right)$ is the unique diffeomorphism for which $p \circ \sigma_{p}$ has constant speed and for which

$$
\int_{0}^{1}\left(\sigma_{p}^{-1}(t)-t\right) d t=0
$$

(By the integrand in (2.7), we mean the unique continuous function $f:[0,1) \rightarrow \mathbf{R}$ for which $f(t) / \mathbf{Z}=\sigma_{p}^{-1}(t)-t$ and for which the value of the integral lies in $[0,1)$.) The reason for requiring (2.7) instead of the basepoint-preserving condition $\sigma_{p}(0)=$ 0 is the naturality implied by the next proposition. However, were we to replace (2.7) with $\sigma_{p}(0)=0$, the other proofs in this paper in which $\sigma_{p}$ enters would not be materially affected. 
Proposition 2.5. The projection $\pi_{\widetilde{\mathcal{M}} \rightarrow \widetilde{\mathcal{M}}^{c}}: \widetilde{\mathcal{M}}_{j}^{\text {int }} \rightarrow \widetilde{\mathcal{M}}_{j}^{\text {int,c }}$ is $S^{1}$-equivariant: $\pi_{\widetilde{\mathcal{M}} \rightarrow \widetilde{\mathcal{M}}^{c}}\left(\tau_{a}(p)\right)=\tau_{a}\left(\pi_{\widetilde{\mathcal{M}} \rightarrow \widetilde{\mathcal{M}}^{c}}(p)\right)$ for all $p \in \widetilde{\mathcal{M}}_{j}^{\text {int }}, a \in S^{1}$.

Proof. For all $p \in \widetilde{\mathcal{M}}_{j}^{\text {int }}$, the unique diffeomorphism $\bar{\sigma}_{p}$ of $S^{1}$ for which $p \circ \bar{\sigma}_{p}$ has constant speed and for which $\bar{\sigma}_{p}(0)=0$ has its inverse given by

$$
\bar{\sigma}_{p}^{-1}(t)=\frac{\int_{0}^{t}|\dot{p}(s)| d s}{\int_{0}^{1}|\dot{p}(s)| d s}=\frac{1}{\ell_{p}} \int_{0}^{t}|\dot{p}(s)| d s .
$$

Every $\sigma \in \operatorname{Diff}_{j}^{+}\left(S^{1}\right)$ for which $p \circ \sigma$ has constant speed satisfies $\sigma^{-1}(t)=\bar{\sigma}^{-1}(t)+$ constant, so

$$
\sigma_{p}^{-1}(t)=\bar{\sigma}_{p}^{-1}(t)-\int_{0}^{1}\left(\bar{\sigma}_{p}^{-1}(s)-s\right) d s
$$

Let $c_{p}=\int_{0}^{1}\left(\bar{\sigma}_{p}^{-1}(s)-s\right)$. Then $\sigma_{p}(t)=\bar{\sigma}_{p}\left(t+c_{p}\right)$, and $S^{1}$-equivariance of $\pi_{\widetilde{\mathcal{M}} \rightarrow \widetilde{\mathcal{M}}^{c}}$ is equivalent to the statement $\sigma_{\tau_{a}(p)}(t)+a=\sigma_{p}(t+a)$, hence to $\bar{\sigma}_{\tau_{a}(p)}\left(t+c_{\tau_{a}(p)}\right)+a=$ $\bar{\sigma}_{p}\left(t+a+c_{p}\right)$. From (2.8) we have $\bar{\sigma}_{\tau_{a}(p)}^{-1}(t)=\bar{\sigma}_{p}^{-1}(t+a)-\bar{\sigma}_{p}^{-1}(a)$. This implies $\bar{\sigma}_{\tau_{a}(p)}(t)=\bar{\sigma}_{p}\left(t+\bar{\sigma}_{p}^{-1}(a)\right)-a$, reducing our equivariance condition to

$$
c_{\tau_{a}(p)}-c_{p}=a-\bar{\sigma}_{p}^{-1}(a) .
$$

Writing $f_{a}(t)=\bar{\sigma}_{\tau_{a}(p)}^{-1}-t$, we have $f_{a}(t)=f_{0}(t+a)+a-\bar{\sigma}_{p}^{-1}(a)$. Integrating both sides over $S^{1}$, (2.10) follows.

Given normalized $C^{j}$ curves $C_{1}, C_{2}$ with $j \geq 2$, the canonical parametrizations $\gamma_{C_{1}}, \gamma_{C_{2}}$ determine a $C^{j}$ diffeomorphism

$$
e_{\left(\kappa_{1}, \kappa_{2}\right)}: S^{1} \times S^{1} \rightarrow C_{1} \times C_{2},
$$

where $\kappa_{i}$ is the normalized curvature function of $C_{i}$. Every normalized $C^{j}$ parametrization $\mu$ of a $\left(C_{1}, C_{2}\right)$-bimorphism $\Sigma$ can be uniquely expressed as a composition

$$
\mu_{\left(p, \kappa_{1}, \kappa_{2}\right)}:=e_{\left(\kappa_{1}, \kappa_{2}\right)} \circ p: S^{1} \rightarrow C_{1} \times C_{2},
$$

where $p: S^{1} \rightarrow S^{1} \times S^{1}$ is a $C^{j}$ nonstop parametrization of a curve in the torus $S^{1} \times S^{1}$; we say that $\left(p, \kappa_{1}, \kappa_{2}\right)$ represents $\Sigma$. The condition that $\Sigma$ is a bimorphism implies that there is a lift of $p$ to a map $\tilde{p}:[0,1] \rightarrow[0,1] \times[0,1]$ with $\tilde{p}(0)=$ $(0,0), \tilde{p}(1)=(1,1)$. Thus $p$ is homotopic to $p^{\Delta}$, and is therefore a $C^{j}$ parametrized internal bimorphism. Conversely, every $C^{j}$ nonstop map $p: S^{1} \rightarrow S^{1} \times S^{1}$ homotopic to $p^{\Delta}$ determines the $C^{j}$ nonstop map $\mu_{\left(p, \kappa_{1}, \kappa_{2}\right)}$. Furthermore, every normalized reparametrization of the image $\Sigma$ of $\mu_{p}$ can be effected by pre-composing $p$ with a diffeomorphism of the circle. Thus, if we define $\widetilde{\mathcal{M}}_{j}\left(C_{1}, C_{2}\right)$, the space of $C^{j}$ parametrized $\left(C_{1}, C_{2}\right)$-h-bimorphisms, to be the set of maps $\left\{e_{\left(\kappa_{1}, \kappa_{2}\right)} \circ p \mid p \in \widetilde{\mathcal{M}}_{j}^{\text {int }}\right\}$; $\widetilde{\mathcal{M}}_{j}^{c}\left(C_{1}, C_{2}\right)$, the space of $C^{j}$ constant-speed $\left(C_{1}, C_{2}\right)$-h-bimorphisms, to be the set of maps $\left\{e_{\left(\kappa_{1}, \kappa_{2}\right)} \circ p \mid p \in \widetilde{\mathcal{M}}_{j}^{\text {int }, c}\right\}$; and $\mathcal{M}_{j}\left(C_{1}, C_{2}\right)$, the space of $C^{j}\left(C_{1}, C_{2}\right)$ h-bimorphisms, to be the quotient of $\mathcal{M}_{j}\left(C_{1}, C_{2}\right)$ by the reparametrization group 
$\operatorname{Diff}_{j}^{+}\left(S^{1}\right)$, then for $1 \leq j \leq \infty$ we obtain basepoint-dependent(!) one-to-one correspondences (in fact, homeomorphisms)

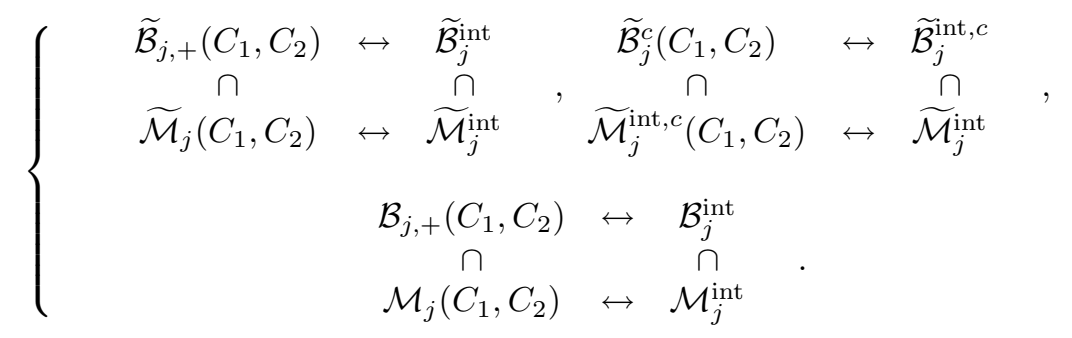

Note that all the spaces above depending on $C_{1}, C_{2}$ really depend only on the (based) shape-equivalences classes of $C_{1}, C_{2}$, in the sense that if $R_{1}, R_{2}$ are similarity transformations of $\mathbf{R}^{2}$, then there are natural identifications of the spaces above with the corresponding spaces for the pair of curves $\left(R_{1}\left(C_{1}\right), R_{2}\left(C_{2}\right)\right)$. Thus we can think of pairs of shapes (or based shapes) as parametrizing the collection of all bimorphisms (or parametrized bimorphisms), where we identify any two bimorphisms (or parametrized bimorphisms) related this way. In view of Lemma 2.3. this motivates the following definition.

Definition 2.6. For $2 \leq j \leq \infty$, we call

$$
\begin{array}{cl}
\widetilde{\mathcal{M}}_{j}:=\widetilde{\mathcal{M}}_{j}^{\mathrm{int}} \times \widetilde{\mathcal{K}}_{j-2} \times \widetilde{\mathcal{K}}_{j-2} & \text { the universal space of } C^{j} \text { parametrized based } \\
& \text { h-bimorphisms; } \\
\widetilde{\mathcal{M}}_{j}^{c}:=\widetilde{\mathcal{M}}_{j}^{\mathrm{int}, c} \times \widetilde{\mathcal{K}}_{j-2} \times \widetilde{\mathcal{K}}_{j-2} & \text { the universal space of } C^{j} \text { constant-speed } \\
\mathcal{M}_{j}^{b}:=\mathcal{M}_{j}^{\mathrm{int}} \times \widetilde{\mathcal{K}}_{j-2} \times \widetilde{\mathcal{K}}_{j-2} & \text { based h-bimorphisms; and } \\
\text { the universal space of } C^{j} \text { based } \text { h-bimorphisms. }
\end{array}
$$

We use similar terminology for the spaces obtained by replacing the symbol $\mathcal{M}$ with the symbol $\mathcal{B}$, just omitting the "h-" from "h-bimorphism". For the spaces of (unbased) bimorphisms and h-bimorphisms, see Definition 3.1; these spaces are not obtained simply by replacing $\widetilde{\mathcal{K}}$ with $\mathcal{K}$.

Remark 2.7. In the definition above it would be more precise, if more awkward, to append the words "of $C^{j}$ curves" to all the space-names. There are situations in which, given curves $C_{1}, C_{2}$ of class $C^{l}$, it is important to consider bimorphisms of class $C^{j}$ with $j<l$, and thus maps of the form $e_{\left(\kappa_{1}, \kappa_{2}\right)} \circ p$ with $e_{\left(\kappa_{1}, \kappa_{2}\right)} \in C^{l}$ and $p \in C^{j}$; see [G1, §3] for example. To simplify the discussion and to avoid even more cumbersome terminology, we do not give names to the corresponding spaces $\widetilde{\mathcal{M}}_{l}^{\text {int }} \times \widetilde{\mathcal{K}}_{j-2} \times \widetilde{\mathcal{K}}_{j-2}$ etc.

The next two theorems (whose proofs are deferred to 4.1 and $\$ 4.2$ respectively) and their corollary state what sorts of manifolds are the spaces above. For the definition of "tame Fréchet manifold" and "tame Fréchet principal bundle" see the paragraph following Remark 5.1 in the Appendix. We adopt Hamilton's convention that a "Fréchet manifold" always means a "smooth Fréchet manifold" ([프, Definition I.4.6.5, pp. 98-99). Below, isomorphism between topological vector spaces always means topological isomorphism.

Theorem 2.8. (a) For $1 \leq j<\infty, \widetilde{\mathcal{M}}_{j}^{\text {int }}$ is a $C^{\infty}$ Banach manifold canonically modeled on $C^{j}\left(S^{1}, \mathbf{R}^{2}\right)$. 
(b) $\widetilde{\mathcal{M}}_{\infty}^{\text {int }}$ is a tame Fréchet manifold canonically modeled on $C^{\infty}\left(S^{1}, \mathbf{R}^{2}\right)$.

(c) For $2 \leq j<\infty, \widetilde{\mathcal{M}}_{j}^{\text {int,c }}$ is a $C^{\infty}$ Banach submanifold of $\widetilde{\mathcal{M}}_{j}^{\text {int }}$, modeled on a subspace of $C^{j}\left(S^{1}, \mathbf{R}^{2}\right)$ isomorphic to $C^{j}\left(S^{1}\right) \oplus \mathbf{R}$.

(d) $\widetilde{\mathcal{M}}_{\infty}^{\text {int,c }}$ is a tame Fréchet submanifold of $\widetilde{\mathcal{M}}_{\infty}^{\text {int }}$ canonically modeled on $C^{\infty}\left(S^{1}\right) \oplus \mathbf{R}$.

(e) For $1 \leq j<\infty$, the space $\mathcal{M}_{j}^{\text {int }}$, endowed with the quotient topology determined by the projection $\pi_{\widetilde{\mathcal{M}} \rightarrow \mathcal{M}}$, is a $C^{0}$ Banach manifold modeled on $C^{j}\left(S^{1}\right)$.

(f) $\mathcal{M}_{\infty}^{\text {int }}$, endowed with the quotient topology determined by the projection $\pi_{\widetilde{\mathcal{M}} \rightarrow \mathcal{M}}$, is a tame Fréchet manifold whose tangent space at each $\Sigma^{\text {int }} \in \mathcal{M}_{\infty}^{\text {int }}$ is canonically isomorphic to $C^{\infty}\left(\Sigma^{\mathrm{int}}\right)$. $\widetilde{\mathcal{M}}_{\infty}^{\text {int }}$ is a tame principal Fréchet $\operatorname{Diff}^{+}\left(S^{1}\right)$ bundle over $\mathcal{M}^{\mathrm{int}}$, and $\widetilde{\mathcal{M}}_{\infty}^{\mathrm{int}, c}$ is a tame principal Fréchet $S^{1}$-bundle over $\mathcal{M}^{\mathrm{int}}$.

When dealing with infinite-dimensional manifolds, whether of Banach or Fréchet type, it is often the case that even locally there is no canonical Banach or Fréchet space in which chart-maps take their values; there is only a class of mutually isomorphic spaces. A manifold $\mathcal{N}$ is modeled canonically on a fixed topological vector space $E$ if and only if for each $p \in \mathcal{N}$ there is a canonical isomorphism between the tangent space $T_{p} \mathcal{N}$ and $E$. (We also say that $\mathcal{N}$ is canonically parallelizable.) Thus in Theorem 2.8, statements (c) and (e) assert something weaker than the other statements.

Except for the statements concerning canonical model spaces, a theorem analogous to Theorem 2.8 is true if we replace "parametrized internal h-bimorphisms" with "embeddings $S^{1} \rightarrow M$ in a fixed homotopy class", where $M$ is any smooth manifold. For the sake of concreteness, we have restricted ourselves to the case $M=S^{1} \times S^{1}$ in stating and proving Theorem 2.8 .

Theorem 2.9. (a) For $0 \leq j<\infty, \widetilde{\mathcal{K}}_{j}$ is a $C^{\infty}$ Banach manifold.

(b) $\widetilde{\mathcal{K}}_{\infty}$ is a tame Fréchet manifold.

Corollary 2.10. $\widetilde{\mathcal{M}}_{\infty}, \widetilde{\mathcal{M}}_{\infty}^{\mathrm{int}, c}$, and $\mathcal{M}_{\infty}^{\mathrm{int}}$ are tame Fréchet manifolds. With $S^{1}$ acting only on the first factor of $\widetilde{\mathcal{M}}_{\infty}^{c}=\widetilde{\mathcal{M}}_{\infty}^{\text {int }, c} \times \widetilde{\mathcal{K}}_{\infty} \times \widetilde{\mathcal{K}}_{\infty}$, the space $\widetilde{\mathcal{M}}_{\infty}^{c}$ is a tame Fréchet principal $S^{1}$-bundle over $\mathcal{M}_{\infty}^{\text {int }}$.

In Theorems 2.8 and 2.9 and their proofs we include the finite- $j$ cases partly to explain why the finite- $j$ spaces are insufficient for the implicit-function-theorem approach that we use to prove Theorem 1.1 (the reason being that $\mathcal{M}_{j}^{\text {int }}$ has no differentiable structure); partly to lay a foundation for the reader who may be able to find, using only the finite- $j$ spaces, a simpler path to an existence/uniqueness theorem for optimal bimorphisms; and partly because the finite- $j$ results may be useful in other contexts and (in the cases of $\mathcal{M}_{j}^{\text {int }}, \widetilde{\mathcal{M}}_{j}^{\text {int,c }}$ and $\widetilde{\mathcal{K}}_{j}$ ) the author has not seen them elsewhere. (However, we note that in [KSMJ, Klassen et al. state an analog of Theorem 2.9(a) for "normalized $L^{2}$ curvature functions"-squareintegrable functions satisfying the first three constraints in our Lemma 4.5 and sketch a proof whose ingredients are essentially the same as those we use to prove Theorem 2.9(a).)

The spaces $\mathcal{K}_{j}$ (and hence $\mathcal{S}_{j}$ ), $0 \leq j \leq \infty$, corresponding to unbased shapes are not manifolds in any natural way consistent with the projections $\widetilde{\mathcal{K}}_{j} \rightarrow \mathcal{K}_{j}$. The problem is that $S^{1}$ does not act freely on $\widetilde{\mathcal{K}}_{j}$; a curvature function with period $1 / n$ for some $n>1$ (corresponding to a curve with $n$-fold rotational symmetry) has 
stabilizer $\mathbf{Z}_{n}$, the cyclic group of order $n$, and the constant curvature function $2 \pi$ (corresponding to the circle) is stabilized by all of $S^{1}$. This would seem to lead to an orbifold-type singularity in the neighborhood of a point in $\mathcal{K}_{j}$ represented by a curvature function with finite symmetry (though the author has not tried to prove this), and a horrible singularity at the point in $\mathcal{K}_{j}$ corresponding to the circle, in every neighborhood of which there exists for every $n$ a curvature function with stabilizer $\mathbf{Z}_{n}$. It does seem likely that the complement in $\mathcal{K}_{j}$ of the set of symmetric curves is a $C^{0}$ manifold for $j<\infty$, and a $C^{\infty}$ manifold for $j=\infty$, and that the pre-images of these subsets in $\widetilde{\mathcal{K}}_{j}$ are principal $S^{1}$-bundles $\left(C^{0}\right.$ for $j<\infty$ and $C^{\infty}$ for $j=\infty$ ).

We now briefly (and only partially) explain why the word "canonically" appears in statement (d) of Theorem 2.8 but not in statement (c), and in statement (f) but not in statement (e); the proof in $\$ 4$ gives a more complete understanding.

Let $p=\left(p_{1}, p_{2}\right) \in \widetilde{\mathcal{M}}_{j}^{\text {int }}(1 \leq j \leq \infty)$. The tangent space $T_{p} \widetilde{\mathcal{M}}_{j}^{\text {int }}$ - the space of "variation vector fields" at $p$-is simply the space of all $C^{j}$ vector fields along the parametrized curve $p$ in the torus $S^{1} \times S^{1}$, and hence is naturally isomorphic to $C^{j}\left(S^{1}, \mathbf{R}^{2}\right)$. If $p \in \widetilde{\mathcal{M}}_{j}^{\text {int }, c}$, then $T_{p} \widetilde{\mathcal{M}}_{j}^{\text {int }, c} \subset T_{p} \widetilde{\mathcal{M}}_{j}^{\text {int }}$ is given by

$$
T_{p} \widetilde{\mathcal{M}}_{j}^{\mathrm{int}, c}=\left\{X=\left(X_{1}, X_{2}\right) \mid X_{i} \in C^{j}\left(S^{1}\right) \text { and } \dot{p} \cdot \dot{X}=\text { constant }\right\} .
$$

Formally, the tangent space to the $S^{1}$ orbit through $p \in \widetilde{\mathcal{M}}_{j}^{\text {int,c }}$ is $\mathbf{R} \dot{p}$, the 1dimensional space of constant multiples of $\dot{p}=\left(\dot{p}_{1}, \dot{p}_{2}\right)$. However, $\left(\dot{p}_{1}, \dot{p}_{2}\right) \notin C^{j}$ unless $p \in \widetilde{\mathcal{M}}_{j+1}^{\text {int }}$, and thus the $S^{1}$ action on $\widetilde{\mathcal{M}}_{j}^{\text {int,c }}$ is not differentiable. If $p \in$ $\widetilde{\mathcal{M}}_{j+1}^{\text {int,c }}$, then the $S^{1}$-orbit of $p$ is a $C^{1}$ submanifold of $\widetilde{\mathcal{M}}_{j}^{\text {int }}$, and we have a natural candidate for the tangent space to $\mathcal{M}_{j}^{\text {int }}$ at $\Sigma=\pi_{\widetilde{\mathcal{M}} \rightarrow \mathcal{M}}(p)$, namely

$$
T_{p} \widetilde{\mathcal{M}}_{j}^{\text {int }, c} / \mathbf{R} \dot{p} .
$$

The isomorphism class of (2.14) is independent of the representative $p$ of $\Sigma$. However, if $j<\infty$, then for general $p \in \widetilde{\mathcal{M}}_{j}^{\text {int,c }}$ the $S^{1}$-orbit of $p$ is not a differentiable object and (2.14) is not even defined. When $j=\infty$ (2.14) does indeed give $T_{\Sigma} \mathcal{M}_{j}^{\mathrm{int}}$.

It is useful to have an alternative characterization of the spaces $T_{p} \widetilde{\mathcal{M}}_{j}^{\text {int,c }}$ for $2 \leq j \leq \infty$, and of $T_{\Sigma} \mathcal{M}_{\infty}^{\text {int }}$ as a subspace of $T_{p} \widetilde{\mathcal{M}}_{\infty}^{\text {int,c }}$ rather than the quotient space (2.14). To facilitate the discussion we define an important invariant of (h-)bimorphisms.

Definition 2.11. For $2 \leq j \leq \infty$ and $p=\left(p_{1}, p_{2}\right) \in \widetilde{\mathcal{B}}_{j}^{\text {int }}$, the slope-angle function of $p$ is the unique function $\theta_{p}: S^{1} \rightarrow[0, \pi / 2]$ such that for all $t \in S^{1}$,

$$
\dot{p}_{1}=L_{p}(t) \cos \theta_{p}(t), \quad \dot{p}_{2}=L_{p}(t) \sin \theta_{p}(t),
$$

where $L_{p}(t)=\sqrt{\dot{p}_{1}(t)^{2}+\dot{p}_{2}(t)^{2}}\left(\equiv \ell_{p}\right.$ if $\left.p \in \widetilde{\mathcal{B}}_{j}^{\text {int }, c}\right)$. Equivalently, $\theta_{p}$ is defined by

$$
\theta_{p}(t):= \begin{cases}\tan ^{-1}\left(\dot{p}_{2}(t) / \dot{p}_{1}(t)\right), & \dot{p}_{1}(t) \neq 0 \\ \pi / 2, & \dot{p}_{1}(t)=0\end{cases}
$$

More generally, for $p \in \widetilde{\mathcal{M}}_{j}^{\text {int }}$ we define the slope-angle function $\bar{\theta}_{p}: S^{1} \rightarrow \mathbf{R} /(2 \pi \mathbf{Z})$ by (2.15). 
Note that $\theta_{p} \in C^{j-1}\left(S^{1}\right)$ if $p \in \widetilde{\mathcal{B}}_{j}^{\text {int }}$ since we have

$$
\dot{\theta}_{p}=\frac{\dot{p}_{1} \ddot{p}_{2}-\dot{p}_{2} \ddot{p}_{1}}{\left(\dot{p}_{1}\right)^{2}+\left(\dot{p}_{2}\right)^{2}} \in C^{j-2}\left(S^{1}\right) \text {. }
$$

Similarly, for all $p \in \widetilde{\mathcal{M}}_{j}^{\text {int }},\left(\bar{\theta}_{p}\right)^{\cdot}$ is still given by the right-hand side of (2.17), so even for $p \in \widetilde{\mathcal{M}}_{j}^{\text {int }}-\widetilde{\mathcal{B}}_{j}^{\text {int }}$ we define $\dot{\theta}_{p}:=\left(\bar{\theta}_{p}\right)^{\text {. }}$.

Note that for $p \in \widetilde{\mathcal{B}}_{j}^{\text {int }}$ and $\sigma \in \operatorname{Diff}_{j}\left(S^{1}\right)$ we have $\theta_{p \circ \sigma}=\theta_{p} \circ \sigma$; the same is true more generally of $\bar{\theta}$. Thus for any $\Sigma \in \widetilde{\mathcal{B}}_{j}^{\text {int }}$ the $\left\{\theta_{p}\right\}_{p \in \pi_{\mathcal{M} \rightarrow \mathcal{M}}^{-1}}(\Sigma)$ determine a welldefined function $\theta_{\Sigma}: \Sigma \rightarrow[0, \pi / 2]$, independent of a choice of parametrization of $\Sigma$. More generally for $\Sigma \in \widetilde{\mathcal{M}}_{j}^{\text {int }}$ we have a well-defined function $\bar{\theta}_{\Sigma}: \Sigma \rightarrow \mathbf{R} /(2 \pi \mathbf{Z})$. The invariant $\theta_{\Sigma}$ (or $\bar{\theta}_{\Sigma}$ ) encodes the relative velocities at which the two factors of $S^{1} \times S^{1}$ are traversed. The quantity $\dot{\theta}(t) / L_{p}(t)\left(\equiv \dot{\theta}(t) / \ell_{p}\right.$ if $\left.p \in \widetilde{\mathcal{M}}_{j}^{\text {int,c }}\right)$ has a direct geometric interpretation: it is the curvature of $\Sigma$ in the flat torus $S^{1} \times S^{1}$ at the point $p(t)$.

Often we will drop the subscript $p$ from $\theta_{p}$, the dependence on $p$ being understood from context. The same goes for the unit tangent and normal vector fields $\mathbf{T}_{p}(t)=$ $\dot{p}(t) /|\dot{p}(t)|=\left(\cos \theta_{p}(t), \sin \theta_{p}(t)\right), \mathbf{N}_{p}(t):=\left(-\sin \theta_{p}(t), \cos \theta_{p}(t)\right)$.

For $p \in \widetilde{\mathcal{M}}_{j}^{\text {int }}$, any variation vector field $X \in T_{p} \widetilde{\mathcal{M}}_{j}^{\text {int }} \cong C^{j}\left(S^{1}, \mathbf{R}^{2}\right)$ can be written as an ordered pair $\left(X_{1}, X_{2}\right)$ with $X_{i} \in C^{j}\left(S^{1}\right)$. $X$ can also be written as a unique linear combination $f \mathbf{N}+\lambda \mathbf{T}$; the coefficient functions $f, \lambda: S^{1} \rightarrow \mathbf{R}$ are given by the dot product of the $C^{j}$ vector field $X$ with the $C^{j-1}$ vector fields $\mathbf{N}, \mathbf{T}$, and hence are $C^{j-1}$. Thus unless $p$ is $C^{j+1}$, the map $T_{p} \widetilde{\mathcal{M}}_{j}^{\text {int }}\left(\cong C^{j}\left(S^{1}, \mathbf{R}^{2}\right)\right) \rightarrow$ $C^{j-1}\left(S^{1}, \mathbf{R}^{2}\right)$ given by $X \mapsto(f, \lambda)$ is an isomorphism if and only if $j=\infty$; for finite $j$ the would-be inverse map $(f, \lambda) \mapsto f \mathbf{N}+\lambda \mathbf{T}$ is not onto.

Focusing on the case $j=\infty$, two variation vector fields $X \in T_{p} \widetilde{\mathcal{M}}_{\infty}^{\text {int }}$ that differ only by the infinitesimal action of the reparametrization group $\operatorname{Diff}^{+}\left(S^{1}\right)$ have the same normal component $f$, and there always exists a 1-parameter subgroup of $\operatorname{Diff}^{+}\left(S^{1}\right)$ whose infinitesimal action carries a given $X$ to one with zero tangent component. In other words, the image of the infinitesimal action of $\operatorname{Diff}^{+}\left(S^{1}\right)$ is precisely the space of tangent vector fields along $p$, so if $\Sigma$ is the h-bimorphism parametrized by $p$, we have a natural candidate (that turns out to be valid rigorously) for $T_{\Sigma} \mathcal{M}_{\infty}^{\text {int }}$, the subspace of normal vector fields

$$
\tilde{T}_{p}^{(0)} \mathcal{M}_{\infty}^{\mathrm{int}}:=\left\{f \mathbf{N}_{p} \in T_{p} \widetilde{\mathcal{M}}_{\infty}^{\mathrm{int}} \mid f \in C^{\infty}\left(S^{1}\right)\right\} \subset T_{p} \widetilde{\mathcal{M}}_{\infty}^{\mathrm{int}} .
$$

However, for $p \in \widetilde{\mathcal{M}}_{\infty}^{\text {int }, c}$, the space $\tilde{T}_{p}^{(0)} \mathcal{M}_{\infty}^{\text {int }}$ is not contained in $T_{p} \widetilde{\mathcal{M}}_{\infty}^{\text {int, },} ;$ normal vector fields $f \mathbf{N}$ do not, in general, correspond to variations of $p$ through constantspeed curves. Expression (2.14) gives us two alternate models for $T_{\Sigma} \mathcal{M}_{\infty}^{\text {int }}$. Since $\dot{\mathbf{T}}=\dot{\theta} \mathbf{N}$ and $\dot{\mathbf{N}}=-\dot{\theta} \mathbf{T}$, we have $\dot{p} \cdot(f \mathbf{N}+\lambda \mathbf{T})=\ell_{p}(\dot{\lambda}-f \dot{\theta})$. Thus from (2.13), $f \mathbf{N}+\lambda \mathbf{T} \in T_{p} \widetilde{\mathcal{M}}_{\infty}^{\text {int,c }}$ if and only if $\dot{\lambda}-f \dot{\theta}$ is constant. Since $\dot{\lambda}$ integrates to zero around the circle, this in turn is equivalent to $\lambda(t)=\lambda_{f}(t)+$ constant, where

$$
\lambda_{f}(t)=\int_{0}^{t}\left(f(s) \dot{\theta}_{p}(s)-\left\langle f \dot{\theta}_{p}\right\rangle\right) d s
$$

and where $\langle g\rangle$ denotes the average value of a function $g$ on the circle,

$$
\langle g\rangle:=\int_{0}^{1} g(t) d t .
$$


Define $\hat{\lambda}_{f}(t)=\lambda_{f}(t)-\left\langle\lambda_{f}\right\rangle$. Then the preceding gives us two ways of splitting $T_{p} \widetilde{\mathcal{M}}{ }_{\infty}^{\text {int,c: }}$

$$
T_{p} \widetilde{\mathcal{M}}_{\infty}^{\mathrm{int}, c}=\tilde{T}_{p}^{(1)} \widetilde{\mathcal{M}}_{\infty}^{\mathrm{int}} \oplus \mathbf{R} \mathbf{T}_{p}=\tilde{T}_{p}^{(2)} \widetilde{\mathcal{M}}_{\infty}^{\mathrm{int}} \oplus \mathbf{R} \mathbf{T}_{p}
$$

where

$$
\begin{aligned}
& \tilde{T}_{p}^{(1)} \mathcal{M}_{\infty}^{\mathrm{int}}:=\left\{f \mathbf{N}_{p}+\lambda_{f} \mathbf{T}_{p} \mid f \in C^{j}\left(S^{1}\right)\right\}, \\
& \tilde{T}_{p}^{(2)} \mathcal{M}_{\infty}^{\mathrm{int}}:=\left\{f \mathbf{N}_{p}+\hat{\lambda}_{f} \mathbf{T}_{p} \mid f \in C^{j}\left(S^{1}\right)\right\} .
\end{aligned}
$$

In each of the splittings in (2.21), the one-dimensional subspace $\mathbf{R T}_{p}$ is the tangent space to the $S^{1}$-orbit at $p$ (generated by translation of the constant-speed parameter $t$ ); thus both $\tilde{T}_{p}^{(1)} \mathcal{M}_{\infty}^{\text {int }}$ and $\tilde{T}_{p}^{(2)} \mathcal{M}_{\infty}^{\text {int }}$ are subspaces of $T_{p} \widetilde{\mathcal{M}}_{\infty}^{\text {int }, c}$ that are natural algebraic models for $T_{\Sigma} \mathcal{M}_{\infty}^{\text {int }}$. However, $\tilde{T}_{p}^{(2)} \mathcal{M}_{\infty}^{\text {int }}$ is more natural geometrically, since it corresponds to a subspace of the space of vector fields along $\Sigma$ that is independent of the choice of representative $p$. Therefore it is the model that we use in the proofs of Theorem 2.8 and [G1, Theorem 5.4] (the stronger version of Theorem 1.11). It is also analytically advantageous to stick with functions of average value zero since their $L^{2}$-norms are bounded by the $L^{2}$ norms of their derivatives; this helps in the proof of [G1, Theorem 5.4]. Of course, both models of $T_{\Sigma} \mathcal{M}_{\infty}^{\text {int }}$ are isomorphic to $\tilde{T}_{p}^{(0)} \mathcal{M}_{\infty}^{\text {int }}$ via $f \mathbf{N}_{p}+\lambda_{f} \mathbf{T}_{p} \leftrightarrow f \mathbf{N}_{p} \leftrightarrow f \mathbf{N}_{p}+\hat{\lambda}_{f} \mathbf{T}_{p}$.

\section{OBJECTIVE FUnCTIONALS AND UNBASED BIMORPHISMS}

In this section, the value of $j$ in Definition 2.6 is not important, so (except in Definition (3.1) we will write simply $\widetilde{\mathcal{M}}$ for $\widetilde{\mathcal{M}}_{j}, \widetilde{\mathcal{K}}$ for $\widetilde{\mathcal{K}}_{j-2}$, etc., where $j \geq 2$ is fixed but arbitrary (and $j=\infty$ is allowed).

Let $\Gamma: \mathbf{R} \rightarrow \mathbf{R}$ be as in (1.1). The grand objective functional determined by $\Gamma$ is the functional

$$
\tilde{J}=\tilde{J}^{\Gamma}: \widetilde{\mathcal{M}} \rightarrow \mathbf{R}
$$

$$
\tilde{J}\left(p ; \kappa_{1}, \kappa_{2}\right)=\int_{0}^{1} \Gamma\left(\frac{\dot{p}_{1}(t) \kappa_{1}\left(p_{1}(t)\right)-\dot{p}_{2}(t) \kappa_{2}\left(p_{2}(t)\right)}{\sqrt{\left(\dot{p}_{1}(t)\right)^{2}+\left(\dot{p}_{2}(t)\right)^{2}}}\right) \sqrt{\left(\dot{p}_{1}(t)\right)^{2}+\left(\dot{p}_{2}(t)\right)^{2}} d t,
$$

where $p=\left(p_{1}, p_{2}\right)$. Writing $\mu=\mu_{\left(p, \kappa_{1}, \kappa_{2}\right)}=\left(\mu_{1}, \mu_{2}\right): S^{1} \rightarrow C_{1} \times C_{2}$, where $C_{i}$ is the normalized curve corresponding to $\kappa_{i}$, letting $\Sigma=\operatorname{image}(\mu) \subset C_{1} \times C_{2}$, and letting $s_{\Sigma}$ denote an arclength parameter along $\Sigma$, we can rewrite (3.1) as the right-hand side of (1.1). From this it is clear that $\tilde{J}$ is invariant under reparametrizations of $\Sigma$, and therefore determines a functional $J: \mathcal{M}^{\text {int }} \times \widetilde{\mathcal{K}} \times \widetilde{\mathcal{K}} \rightarrow \mathbf{R}$. Thus we can think of $\kappa_{1}, \kappa_{2}$ as parameters in (3.1), and view the space of pairs of based shapes, $\widetilde{\mathcal{S}} \times \widetilde{\mathcal{S}}(\longleftrightarrow \widetilde{\mathcal{K}} \times \widetilde{\mathcal{K}}$, by Lemma 2.3), as parametrizing a family of functionals $\left\{\tilde{J}_{\left(C_{1}, C_{2}\right)}\right\}_{\left(C_{1}, C_{2}\right) \in \tilde{\mathcal{S}} \times \widetilde{\mathcal{S}}}$, defined on the fixed space $\widetilde{\mathcal{M}}^{\text {int }}$, and similarly a family of functionals $\left\{J_{\left(C_{1}, C_{2}\right)}\right\}$ defined on $\mathcal{M}^{\text {int }}$. (Here of course $\tilde{J}_{\left(C_{1}, C_{2}\right)}(p)=\tilde{J}\left(p ; \kappa_{1}, \kappa_{2}\right)$, etc. for $J_{\left(C_{1}, C_{2}\right)}$, where $\kappa_{i}$ is the normalized curvature function of $C_{i}$.)

Although we have defined $\tilde{J}$ on the space of (parametrized, based) h-bimorphisms, and it will be important later that this space is a manifold, we are interested only in the restriction of $\tilde{J}$ to the space of (parametrized, based) bimorphisms-no "h". Because of (2.5) and the parametrization-invariance of $\tilde{J}_{\left(\kappa_{1}, \kappa_{2}\right)}$, minima of $J_{\left(\kappa_{1}, \kappa_{2}\right)}$ 
on $\mathcal{B}^{\text {int }}$ are precisely the projections of minima of $\tilde{J}_{\left(\kappa_{1}, \kappa_{2}\right)}$ on $\widetilde{\mathcal{B}}^{\text {int }, c}$. Thus, for example, if $J_{\left(\kappa_{1}, \kappa_{2}\right)}$ achieves a minimum at a unique point $\Sigma \in \mathcal{B}^{\text {int }}$, then $\tilde{J}_{\left(\kappa_{1}, \kappa_{2}\right)}^{c}$ will have a circle's worth of minima, the constant-speed parametrizations of $\Sigma$.

It should be clear now why only pairs of based shapes parametrize the family of objective functionals given by $J$ : any parametrization of a curve implicitly involves a choice of basepoint. However, were the set of $J_{\left(C_{1}, C_{2}\right)}$-minimizing $\left(C_{1}, C_{2}\right)$ bimorphisms to depend on choices of basepoints of $C_{1}$ and $C_{2}$, then $\tilde{J}$ would be a poor objective functional. More precisely, the set of minimizers should be equivariant under a change of basepoint: if $C_{1}^{\prime}, C_{2}^{\prime}$ are based curves representing the same unbased curves as $C_{1}, C_{2}$, then the change-of-basepoint operation should give us an identification of $\mathcal{B}\left(C_{1}, C_{2}\right)$ with $\mathcal{B}\left(C_{1}^{\prime}, C_{2}^{\prime}\right)$, and that operation should give us a "dictionary" between the functionals $J_{\left(C_{1}, C_{2}\right)}$ and $J_{\left(C_{1}^{\prime}, C_{2}^{\prime}\right)}$; in particular it should carry a minimizer of one functional to a minimizer of the other.

It is easy to see geometrically that $\tilde{J}$ has this property (see TOG]), in addition to symmetry under interchange of the two plane curves: $\tilde{J}\left(\left(p_{1}, p_{2}\right) ; \kappa_{1}, \kappa_{2}\right)=$ $\tilde{J}\left(\left(p_{2}, p_{1}\right) ; \kappa_{2}, \kappa_{1}\right)$. However, $S^{1}$ acts on each of the three factors of $\widetilde{\mathcal{M}}^{\text {int }, c} \times \widetilde{\mathcal{K}} \times \widetilde{\mathcal{K}}$, leading to three actions on the product, and it is easy to confuse the precise roles of the different actions. In the remainder of this section we elucidate these roles and define the spaces of unbased (h-)bimorphisms.

For $a \in S^{1}$ define $\rho_{0}(a), \rho_{1}(a)$, and $\rho_{2}(a): \widetilde{\mathcal{M}}^{\text {int }, c} \times \widetilde{\mathcal{K}} \times \widetilde{\mathcal{K}} \rightarrow \widetilde{\mathcal{M}}^{\text {int }, c} \times \widetilde{\mathcal{K}} \times \widetilde{\mathcal{K}}$ as follows:

$$
\begin{aligned}
& \rho_{0}(a)\left(\left(p_{1}, p_{2}\right), \kappa_{1}, \kappa_{2}\right)=\left(\left(\tau_{a}\left(p_{1}\right), \tau_{a}\left(p_{2}\right)\right), \kappa_{1}, \kappa_{2}\right), \\
& \rho_{1}(a)\left(\left(p_{1}, p_{2}\right), \kappa_{1}, \kappa_{2}\right)=\left(\left(p_{1}-a, p_{2}\right), \tau_{a}\left(\kappa_{1}\right), \kappa_{2}\right), \\
& \rho_{2}(a)\left(\left(p_{1}, p_{2}\right), \kappa_{1}, \kappa_{2}\right)=\left(\left(p_{1}, p_{2}-a\right), \kappa_{1}, \tau_{a}\left(\kappa_{2}\right)\right)
\end{aligned}
$$

(see (2.2); here $p_{i}-a$ means the function $t \mapsto p_{i}(t)-a$ ). Observe that $\mu_{\rho_{0}(a)\left(p, \kappa_{1}, \kappa_{2}\right)}$ $=\tau_{a}\left(\mu_{\left(p, \kappa_{1}, \kappa_{2}\right)}\right)$; thus if $C_{1}, C_{2}$ are the normalized curves corresponding to $\kappa_{1}, \kappa_{2}$, and $\Sigma=$ image $\left(\mu_{\left(p, \kappa_{1}, \kappa_{2}\right)}\right) \subset C_{1} \times C_{2}$, then $\mu_{\rho_{0}(a)\left(p, \kappa_{1}, \kappa_{2}\right)}$ is merely a reparametrization of the same curve $\Sigma$ in the same torus $C_{1} \times C_{2}$.

However, for $i=1,2$, the action $\rho_{i}(a)$ changes the basepoint of $C_{i}$ and, under the correspondence $\widetilde{\mathcal{K}} \leftrightarrow \widetilde{\mathcal{S}}$, simultaneously translates and rotates $C_{i}$ to superimpose the basepoint on the origin and align the initial tangent vector with the positive $x$ axis. Thus for $a \neq 0$ the curve corresponding to $\tau_{a}(\kappa)$ is not the curve corresponding to $\kappa$ (though it has the same size and shape). In fact, as one can easily compute, if we write $\mu_{\left(p, \kappa_{1}, \kappa_{2}\right)}=\left(\mu_{1}, \mu_{2}\right)$ then

$$
\mu_{\rho_{1}(a)\left(p, \kappa_{1}, \kappa_{2}\right)}=\left(R_{a, \kappa_{1}} \circ \mu_{1}, \mu_{2}\right),
$$

where $R_{a, \kappa_{1}}$ is as in (2.3). Thus the image of $\mu_{\rho_{1}(a)\left(p, \kappa_{1}, \kappa_{2}\right)}$ lies not in $C_{1} \times C_{2}$, but in the isometric torus $R_{a, \kappa_{1}}\left(C_{1}\right) \times C_{2}$; furthermore (3.2) gives an explicit oneto-one correspondence between $\widetilde{\mathcal{B}}\left(C_{1}, C_{2}\right)$ and $\widetilde{\mathcal{B}}\left(R_{a, \kappa_{1}}\left(C_{1}\right), C_{2}\right)$. Had we defined $\rho_{1}(a)$ more naively by $\left(\left(p_{1}, p_{2}\right), \kappa_{1}, \kappa_{2}\right) \mapsto\left(\left(p_{1}, p_{2}\right), \tau_{a}\left(\kappa_{1}\right), \kappa_{2}\right)$ then this would not have been the case. In other words, the "naive" action of $S^{1}$ on the second factor of $\widetilde{\mathcal{M}}^{\text {int }, c} \times \widetilde{\mathcal{K}} \times \widetilde{\mathcal{K}}$ does not provide the change-of-basepoint dictionary induced by the geometric identification of $R_{a, \kappa_{1}}\left(C_{1}\right)$ with $C_{1}$. Of course, analogous statements hold for $\rho_{2}$. 
Letting $\mathrm{j}\left(p, \kappa_{1}, \kappa_{2}\right)(t)$ denote the integrand of (3.1) , one can also easily compute that

and

$$
\mathrm{j}\left(\rho_{0}(a)\left(p, \kappa_{1}, \kappa_{2}\right)\right)(t)=\mathrm{j}\left(p, \kappa_{1}, \kappa_{2}\right)(t+a)
$$

so that $\tilde{J}$ is invariant under all three actions. Equations (3.3) also show that

$$
\tilde{J}_{\left(C_{1}, C_{2}\right)}(p)=\tilde{J}_{\left(R_{a, \kappa_{1}}\left(C_{1}\right), C_{2}\right)}\left(p_{1}-a, p_{2}\right)=\tilde{J}_{\left(C_{1}, R_{a, \kappa_{2}}\left(C_{2}\right)\right)}\left(p_{1}, p_{2}-a\right) .
$$

Thus if $p \in \mathcal{B}^{\text {int }}$ minimizes $\left.\tilde{J}_{\left(C_{1}, C_{2}\right)}\right|_{\mathcal{B}^{\text {int }}}$, then $\left(p_{1}-a, p_{2}\right)$ minimizes $\left.\tilde{J}_{\left(R_{a, \kappa_{1}}\left(C_{1}\right), C_{2}\right)}\right|_{\mathcal{B}^{\text {int }}}$, and $\left(p_{1}, p_{2}-a\right)$ minimizes $\left.\tilde{J}_{\left(C_{1}, R_{a, \kappa_{2}}\left(C_{2}\right)\right)}\right|_{\mathcal{B}^{\text {int }}}$. This is the desired equivariance of minimizers with respect to a change of basepoint of $C_{1}, C_{2}$, and it confirms that the value of the minimum of $\tilde{J}_{\left(C_{1}, C_{2}\right)}$ (if it exists) depends only on the (unbased) shape-equivalence classes of $C_{1}$ and $C_{2}$.

The three actions $\rho_{0}, \rho_{1}, \rho_{2}$ all commute and hence give rise to an action $\rho$ of $S^{1} \times S^{1} \times S^{1}$, leading to the correct definition of the spaces of (unbased) bimorphisms and h-bimorphisms:

Definition 3.1. For $2 \leq j \leq \infty$, we call $\mathcal{M}_{j}:=\left(\widetilde{\mathcal{M}}_{j}^{\text {int }, c} \times \widetilde{\mathcal{K}}_{j-2} \times \widetilde{\mathcal{K}}_{j-2}\right) /\left(S^{1} \times S^{1} \times\right.$ $\left.S^{1}\right)$ the universal space of $C^{j} h$-bimorphisms and $\mathcal{B}_{j}:=\left(\widetilde{\mathcal{B}}_{j}^{\text {int,c }} \times \widetilde{\mathcal{K}}_{j-2} \times \widetilde{\mathcal{K}}_{j-2}\right) /\left(S^{1} \times\right.$ $\left.S^{1} \times S^{1}\right)$ the universal space of $C^{j}$ bimorphisms, where $S^{1} \times S^{1} \times S^{1}$ acts by $\rho$, not by $\tau \times \tau \times \tau$.

Because $\rho_{0}$ involves only the first factor of $\widetilde{\mathcal{M}}^{\text {int }} \times \widetilde{\mathcal{K}} \times \widetilde{\mathcal{K}}$ and commutes with $\rho_{1}$ and $\rho_{2}$,

$$
\begin{aligned}
\left(\widetilde{\mathcal{M}}^{\text {int }} \times \widetilde{\mathcal{K}} \times \widetilde{\mathcal{K}}\right) /\left(S^{1} \times S^{1} \times S^{1}\right) & =\left(\left(\widetilde{\mathcal{M}}^{\text {int }} / S^{1}\right) \times \widetilde{\mathcal{K}} \times \widetilde{\mathcal{K}}\right) /\left(S^{1} \times S^{1}\right) \\
& =\left(\mathcal{M}^{\text {int }} \times \widetilde{\mathcal{K}} \times \widetilde{\mathcal{K}}\right) /\left(S^{1} \times S^{1}\right),
\end{aligned}
$$

where the action of $S^{1} \times S^{1}$ on $\mathcal{M}^{\text {int }} \times \widetilde{\mathcal{K}} \times \widetilde{\mathcal{K}}$ is $\left(\bar{\rho}_{1}, \bar{\rho}_{2}\right)$, the action induced $\left(\rho_{1}, \rho_{2}\right)$ after taking the quotient by $\rho_{0}$ (i.e. $\bar{\rho}_{1}(a)\left(\left[\left(p_{1}, p_{2}\right)\right], \kappa_{1}, \kappa_{2}\right)=\left(\left[\left(p_{1}-a, p_{2}\right)\right], \kappa_{1}, \kappa_{2}\right)$, etc. for $\bar{\rho}_{2}$, where [ ] denotes equivalence class determined by $\left.\rho_{0}\right)$. Because the actions $\rho_{1}, \rho_{2}$ commute with each other, one can also write the space (3.5) as $\left(\left(\left(\mathcal{M}^{\text {int }} \times \widetilde{\mathcal{K}}\right) / S^{1}\right) \times \widetilde{\mathcal{K}}\right) / S^{1}$, modding out first by $\bar{\rho}_{1}$, then by the residual action of $\bar{\rho}_{2}$. However, because $\rho_{1}$ and $\rho_{2}$ involve the first factor of $\widetilde{\mathcal{M}}^{\text {int }} \times \widetilde{\mathcal{K}} \times \widetilde{\mathcal{K}}$ as well as the second and third, the quotient space (3.5) is not simply $\mathcal{M}^{\text {int }} \times\left(\widetilde{\mathcal{K}} / S^{1}\right) \times\left(\widetilde{\mathcal{K}} / S^{1}\right)=$ $\mathcal{M}^{\text {int }} \times \mathcal{K} \times \mathcal{K}$.

Since $\tilde{J}$ is invariant under each $\rho_{i}$ (hence under $\rho$ ), there is an induced functional $\bar{J}$ on the quotient space $\mathcal{B}$. The fact that $\mathcal{B}$ is not simply $\mathcal{B}^{\text {int }} \times \mathcal{K} \times \mathcal{K}$ is the reason that we cannot use the space of pairs of unbased shapes to parametrize a good family of objective functionals on the space of unbased internal bimorphisms.

\section{The proofs of Theorems 2.8 and 2.9}

In this section we prove Theorems 2.8 and 2.9 Our proofs of Theorem 2.9. parts (d) and (f) of Theorem 2.8, and (in [G1]) Theorem 1.1 rely on the NashMoser Inverse Function Theorem. This theorem, its relevant corollaries, and the definitions of terms used below related to "tame" objects, are given in the Appendix. Facts listed there under Remark 5.1 will be used so often in the proofs in this section that we will usually use them without explicit mention. Often this general 
"tame calculus" enables us to prove that certain explicitly-defined maps are tamely smooth, but proving that the inverses of their derivatives are tamely smooth requires actual estimates.

In this section we abbreviate the $C^{n}$ norms on $C^{\infty}\left(S^{1}\right)$ and $C^{\infty}\left(S^{1}, \mathbf{R}^{2}\right)$ as \|\|$_{n}$.

\subsection{Manifolds of internal h-bimorphisms.}

Proof of Theorem 2.8. (a) and (b). For $0 \leq j \leq \infty, 0 \leq n \leq j$, and $\epsilon>0$ define

$$
U_{j}^{n, \epsilon}=\left\{X \in C^{j}\left(S^{1}, \mathbf{R}^{2}\right) \mid\|X\|_{n}<\epsilon\right\},
$$

and for all $p \in \widetilde{\mathcal{M}}_{j}^{\text {int }}$ define $\Theta_{p}: U_{j}^{0,1} \rightarrow \widetilde{\mathcal{M}}_{j}^{\text {int }}$ by $\Theta_{p}(X)=p+X$. It is easy to check that the collection $\left\{\left(\Theta_{p}\left(U_{j}^{0,1}\right), \Theta_{p}^{-1}\right)\right\}_{p \in \widetilde{\mathcal{M}}_{j}^{\text {int }}}$ forms a smooth atlas (of Banach type if $j<\infty$, and of tame Fréchet type if $j=\infty$; see $[\mathrm{H}]$, Definition I.4.1.1 and paragraph II.2.3).

(c) For $0 \leq j \leq \infty$ let $C_{0}^{j}\left(S^{1}\right)=\left\{f \in C^{j}\left(S^{1}\right) \mid\langle f\rangle=0\right\}$ (notation as in (2.20)).

Given $p \in \widetilde{\mathcal{M}}_{j}^{\text {int }}$ we have a natural identification $T_{p} \widetilde{\mathcal{M}}_{j}^{\text {int }} \cong C^{j}\left(S^{1}, \mathbf{R}^{2}\right)$. Define $F: \widetilde{\mathcal{M}}_{j}^{\text {int }} \rightarrow C_{0}^{j-2}\left(S^{1}\right)$ by $F(p)=\frac{1}{2}(\dot{p} \cdot \dot{p})=\dot{p} \cdot \ddot{p}$. Note that $F$ is $C^{\infty}$ (the third derivative of $F \circ \Theta_{p}: U_{j}^{0,1} \rightarrow C^{\infty}\left(S^{1}, \mathbf{R}^{2}\right)$ is identically zero) and that $F^{-1}(0)=$ $\widetilde{\mathcal{M}}_{j}^{\text {int,c }}$. We claim that 0 is a regular value of $F$ in the Banach-manifold sense; i.e. that for all $p \in \widetilde{\mathcal{M}}_{j}^{\text {int,c }}$ (i) $\left.D F\right|_{p}: C^{j}\left(S^{1}, \mathbf{R}^{2}\right) \rightarrow C_{0}^{j-2}\left(S^{1}\right)$ is surjective, and (ii) $C_{0}^{j-2}\left(S^{1}\right)$ is isomorphic to a topological direct summand of $C^{j}\left(S^{1}, \mathbf{R}^{2}\right)$ with complement $\operatorname{ker}\left(\left.D F\right|_{p}\right)$.

Let $p \in \widetilde{\mathcal{M}}_{j}^{\text {int,c }}$. Locally there exist smooth vector fields along $p$ whose inner product with $\dot{p}$ is everywhere positive, and we can patch such locally-defined vector fields together with a smooth partition of unity to obtain a smooth "pseudotangent" vector field $V$ along $p$ with $\dot{p}(t) \cdot V(t)>0$ for all $t$. For $f \in C^{j}\left(S^{1}\right)$, the vector field $f V$ along $p$ lies in $C^{j}\left(S^{1}, \mathbf{R}^{2}\right)$ (this would fail with $V$ replaced by $\mathbf{T}_{p}$, which in general is only $C^{j-1}$ ).

For surjectivity of $\left.D F\right|_{p}$, it suffices to check that for all $g \in C_{0}^{j-2}\left(S^{1}\right)$ there exists $f \in C^{j}\left(S^{1}\right)$ such that $\left.D F\right|_{p}(f V)=g$. In general we have $\left.D F\right|_{p}(X)=(\dot{p}, \dot{X})^{\cdot}$, so we seek a solution of $\left(\dot{p},(f V)^{\circ}\right)^{\circ}=g$. Since the average value of $g$ is 0 , the formula

$$
\omega_{g}(t):=\int_{0}^{t} g(u) d u
$$

yields a well-defined function $\omega_{g} \in C^{j-1}\left(S^{1}\right)$. Thus it suffices to show that for some constant $c$ the ODE

$$
\dot{f}+\frac{(\dot{p}, \dot{V})}{(\dot{p}, V)} f=\frac{\omega_{g}+c}{(\dot{p}, V)}
$$

admits a solution $f \in C^{j}\left(S^{1}\right)$. Equivalently, regarding all functions in (4.2) as period-1 functions defined on the real line, it suffices to show that (4.2) admits a $C^{j}$ solution of period 1. The solutions of (4.2) on $\mathbf{R}$ are given by

$$
\left.f(t)=I(t)^{-1}\left(f(0)+\int_{0}^{t} I(s) \frac{\omega_{g}+c}{(\dot{p}, V)}(s) d s\right)\right),
$$

where $I(t)=\exp \left(\int_{0}^{t} \frac{(\dot{p}, \dot{V})}{(\dot{p}, V)}(s) d s\right)$. Since $p$ is $C^{j}$, the coefficient of $f$ in (4.2) and the right-hand side of (4.2) are $C^{j-1}$, so all solutions $f$ are $C^{j}$, and it suffices to show 
that for some $c$ one of them has period 1. From (4.3), "period 1" is equivalent to

$$
I(1)^{-1}\left(f(0)+\int_{0}^{1} I(s) \frac{\omega_{g}}{(\dot{p}, V)}(s) d s+c \int_{0}^{1} \frac{I}{(\dot{p}, V)}(s) d s\right)=f(0) .
$$

Since both $I$ and $(\dot{p}, V)$ are strictly positive, so is the multiplier of $c$ in (4.4). Hence for any choice of $f(0)$, a unique $c$ satisfying (4.4) exists. In particular, $\left.D F\right|_{p}$ is surjective.

Define $\Lambda_{p}: C_{0}^{j-2}\left(S^{1}\right) \rightarrow C^{j}\left(S^{1}, \mathbf{R}^{2}\right)$ by $\Lambda_{p}(g)=\rho(g) V$, where $(\rho(g), c)$ is the unique solution of the system (4.2), (4.4) with $\rho(g)(0)=0$; specifically

$$
\begin{aligned}
\rho(g)(t)= & \frac{1}{I(t) \int_{0}^{1} \frac{I}{(\dot{p}, V)}(s) d s}\left\{\left(\int_{0}^{1} \frac{I}{(\dot{p}, V)}(s) d s\right)\left(\int_{0}^{t} \frac{I \omega_{g}}{(\dot{p}, V)}(s) d s\right)\right. \\
& \left.-\left(\int_{0}^{t} \frac{I}{(\dot{p}, V)}(s) d s\right)\left(\int_{0}^{1} \frac{I \omega_{g}}{(\dot{p}, V)}(s) d s\right)\right\} .
\end{aligned}
$$

By construction, $\Lambda_{p}$ is a right-inverse to $\left.D F\right|_{p}$, and since $V$ is $C^{\infty}$ (4.5) shows that $\Lambda_{p}$ is bounded as a map $C_{0}^{j-2}\left(S^{1}\right) \rightarrow C^{j}\left(S^{1}, \mathbf{R}^{2}\right)$. Hence the image of $\Lambda_{p}$ is a closed subspace of $C^{j}\left(S^{1}, \mathbf{R}^{2}\right)$ with complement the closed subspace $\operatorname{ker}\left(\left.D F\right|_{p}\right)$.

Thus 0 is a regular value of $F$, so by the Regular Value Theorem for Banach manifolds $F^{-1}(0)$ is a smooth submanifold of $\widetilde{\mathcal{M}}_{j}^{\text {int }}$. It remains only to show that for $p \in F^{-1}(0), \operatorname{ker}\left(\left.D F\right|_{p}\right) \cong C^{j}\left(S^{1}\right) \oplus \mathbf{R}$.

Let $\mathrm{J} V$ be the $C^{j}$ vector field along $p$ obtained by rotating $V$ counterclockwise at each point by $\pi / 2$. Every $X \in C^{j}\left(S^{1}, \mathbf{R}^{2}\right)$ can be written uniquely as $f_{1} \mathrm{~J} V+f_{2} V$, where $f_{1}, f_{2} \in C^{j}\left(S^{1}\right)$. We have $X \in \operatorname{ker}\left(\left.D F\right|_{p}\right)$ iff $(\dot{p}, \dot{X})$ is constant; equivalently, iff

$$
\dot{f}_{2}+\frac{(\dot{p}, \dot{V})}{(\dot{p}, V)} f_{2}=\frac{c-\left(\dot{p},\left(f_{1} \mathrm{~J} V\right)^{\cdot}\right)}{(\dot{p}, V)} .
$$

The same analysis as above shows that for each $f_{1}$ and each $a \in \mathbf{R}$ there is a unique pair $\left(f_{2}, c\right)$ such that $f_{2}(0)=a$ and (4.6) is satisfied. Writing $f_{2}=\lambda_{V}\left(f_{1}, a\right)$, the map $\left(f_{1}, a\right) \mapsto f_{1} \mathrm{~J} V+\lambda_{V}\left(f_{1}, a\right) V$ then gives an isomorphism $C^{j}\left(S^{1}\right) \oplus \mathbf{R} \rightarrow$ $\operatorname{ker}\left(\left.D F\right|_{p}\right)$.

(d) Analogously to the case $j<\infty$, define $F: \widetilde{\mathcal{M}}_{\infty}^{\text {int }} \rightarrow C^{\infty}\left(S^{1}\right)$ by $F(p)=$ $\frac{1}{2}(\dot{p} \cdot \dot{p})^{\text {. }}$. Then $F$ is a tamely smooth map of Fréchet manifolds, and $\widetilde{\mathcal{M}}_{\infty}^{\text {int, },}=$ $F^{-1}(0)$. Our strategy below is to establish that 0 is a tame regular value of $F$, from which can conclude that $\widetilde{\mathcal{M}}_{\infty}^{\text {int,c }}$ is a tame Fréchet submanifold of $\widetilde{\mathcal{M}}_{\infty}^{\text {int }}$. However, to establish that this submanifold is modeled canonically on $C^{\infty}\left(S^{1}\right) \oplus \mathbf{R}$, we bypass Theorem 5.5 and directly produce a submanifold atlas for $\widetilde{\mathcal{M}}_{\infty}^{\text {int,c }}$ (see $[\mathbf{H}$, Definition I.4.2.1, p. 87). This extra detail adds only three sentences at the end of this (rather long) proof; essentially all the work is in showing that 0 is a tame regular value.

Let $p \in \widetilde{\mathcal{M}}_{\infty}^{\text {int,c }}$. Then, as in (c), we have an isomorphism $C^{\infty}\left(S^{1}\right) \oplus \mathbf{R} \rightarrow$ $\operatorname{ker}\left(\left.D F\right|_{p}\right),(f, a) \mapsto f \mathbf{N}_{p}+\lambda_{p}(f, a) \mathbf{T}_{p}$, where $\lambda_{p}=\lambda_{\mathbf{T}_{p}}$ in the notation used in (c). Because we are now taking $V=\mathbf{T}_{p}, J V=\mathbf{N}_{p}$, the formulas of part (c) simplify considerably. Since $\dot{\mathbf{T}}_{p}=\dot{\theta}_{p} \mathbf{N}_{p}$ and $\dot{\mathbf{N}}_{p}=-\dot{\theta}_{p} \mathbf{T}_{p}$, we have $I(t) \equiv 1$ and $\lambda_{p}(f, a)=a+\lambda_{p}(f)$, where for all $q \in \widetilde{\mathcal{M}}^{\text {int }}$ (not just $\widetilde{\mathcal{M}}^{\text {int }, c}$ ) we define

$$
\lambda_{q}(f)(t):=\int_{0}^{t}\left(f \dot{\theta}_{q}-\left\langle f \dot{\theta}_{q}\right\rangle\right)(s) d s .
$$


It will be more convenient to use a slightly different isomorphism $C^{\infty}\left(S^{1}\right) \oplus \mathbf{R} \rightarrow$ $\operatorname{ker}\left(\left.D F\right|_{p}\right)$, namely

$$
\begin{aligned}
H_{p}: C^{\infty}\left(S^{1}\right) \oplus \mathbf{R} & \rightarrow \operatorname{ker}\left(\left.D F\right|_{p}\right), \\
H_{p}(f, a) & =f \mathbf{N}_{p}+\hat{\lambda}_{p}(f, a) \mathbf{T}_{p},
\end{aligned}
$$

where

$$
\hat{\lambda}_{p}(f, a):=a+\hat{\lambda}_{p}(f)
$$

and

$$
\hat{\lambda}_{p}(f):=\lambda_{p}(f)-\left\langle\lambda_{p}(f)\right\rangle .
$$

Some useful invariance and equivariance properties enjoyed by the family of maps $\hat{\lambda}$ (but not by $\lambda$ ) are that for all $q \in \widetilde{\mathcal{M}^{\text {int }}}, f \in C^{\infty}\left(S^{1}\right), a \in \mathbf{R}$, and $\sigma \in \operatorname{Diff}^{+}\left(S^{1}\right)$, we have

$$
\left\langle\hat{\lambda}_{q}(f)\right\rangle=0, \quad\left\langle\hat{\lambda}_{q}(f, a)\right\rangle=a
$$

and

$$
\hat{\lambda}_{p \circ \sigma}(f \circ \sigma)=\hat{\lambda}_{p}(f) \circ \sigma .
$$

For later use, we note that for each $j \geq 0$

$$
\left\|\hat{\lambda}_{p}(f, a)\right\|_{j} \leq C\left(\|f\|_{\max \{j-1,0\}}+|a|\right) .
$$

Here and below, $C$ denotes a continually updated constant that may depend on $p$ and on which norm is being taken, but is otherwise universal; for constants that depend on additional parameters we write $C(\cdot)$.

Analogously to the situation in (c), we have a linear map $\Lambda_{p}: C_{0}^{\infty}\left(S^{1}\right) \rightarrow$ $C^{\infty}\left(S^{1}, \mathbf{R}^{2}\right)$ that is a continuous right-inverse of $\left.D F\right|_{p}$ :

$$
\begin{aligned}
\Lambda_{p}(g) & =\rho(g) \mathbf{T}_{p}, \\
\text { where } \quad \rho(g)(t) & =\frac{1}{\ell_{p}} \int_{0}^{t}\left(\omega_{g}-\left\langle\omega_{g}\right\rangle\right)(s) d s .
\end{aligned}
$$

Note that $C_{0}^{\infty}\left(S^{1}\right)$ is tame since it is a tame direct summand of $C^{\infty}\left(S^{1}\right)$ ([]ㅡ, Lemma II.1.3.3, p. 136).

Define maps

$$
\begin{gathered}
\tilde{\Phi}_{p}: U_{\infty}^{2, \epsilon} \times(-\epsilon, \epsilon) \rightarrow C^{\infty}\left(S^{1}, S^{1} \times S^{1}\right), \\
(f, a) \mapsto p+H_{p}(f, a), \\
\tilde{\Psi}_{p}: U_{\infty}^{2, \epsilon} \times(-\epsilon, \epsilon) \times\left(U_{\infty}^{0, \epsilon} \cap C_{0}^{\infty}\left(S^{1}\right)\right)(f, a, g) \mapsto\left(\tilde{\Phi}_{p}(f, a)+\Lambda_{p}(g)\right) \circ \sigma_{\tilde{\Phi}_{p}(f, a)}
\end{gathered}
$$

(see (2.6) ). Note that $\tilde{\Psi}_{p}(f, a, 0)$ is simply $\pi_{\widetilde{\mathcal{M}} \rightarrow \widetilde{\mathcal{M}}^{c}}\left(\tilde{\Phi}_{p}(f, a)\right)$. Thus, heuristically, $\tilde{\Psi}_{p}$ is a "normal exponential map" based on $\widetilde{\mathcal{M}}_{\infty}^{\text {int,c }}$ (which we do not yet know is a submanifold), expressed in terms of $(f, a)$ - which eventually will be local coordi-

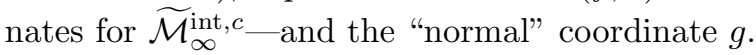

We will see that, after shrinking the domains of the maps $\tilde{\Psi}_{p}$ to sufficiently small open sets $V(p)$, the charts $\left(V(p),\left.\tilde{\Psi}_{p}^{-1}\right|_{V(p)}\right)$ form a submanifold atlas for $\widetilde{\mathcal{M}}_{\infty}^{\text {int,c }} \subset \widetilde{\mathcal{M}}_{\infty}^{\text {int }}$. First, we claim that by taking $\epsilon=\epsilon(p)<1$ small enough we can ensure that the image of $\tilde{\Psi}_{p}$ lies in the domain of the chart-map $\Theta_{p}^{-1}$. To see this, for the remainder of this proof fix $p \in \widetilde{\mathcal{M}}_{\infty}^{\text {int,c }}$ and note that the $C^{0}$ norm of $H_{p}(f, a)+\Lambda_{p}(g)$ can then be bounded purely in terms of $\|f\|_{0},|a|$, and $\|g\|_{0}$. Hence 
if $\epsilon$ is small enough then $\left\|H_{p}(f, a)+\Lambda_{p}(g)\right\|_{0}<1 / 2$; thus $\tilde{\Phi}_{p}(f, a)+\Lambda_{p}(g)=p+Y$, where $Y=H_{p}(f, a)+\Lambda_{p}(g) \in C^{\infty}\left(S^{1}, \mathbf{R}^{2}\right)$ has $C^{0}$-norm less than $1 / 2$.

Notation 4.1. Define $\|(f, a)\|_{n}:=\|f\|_{n}+|a|$ for $(f, a) \in C^{\infty}\left(S^{1}\right) \oplus \mathbf{R}$ and $\|(f, a, g)\|_{n}$ $:=\|(f, a)\|_{n}+\|g\|_{\max \{n-2,0\}}$ for $(f, a, g) \in C^{\infty}\left(S^{1}\right) \oplus \mathbf{R} \oplus C_{0}^{\infty}\left(S^{1}\right)$. (No harm would be done to the proof by instead using $\|g\|_{n}$ in the latter norm, but the choice above turns out to be more naturally suited to our situation.) We will use both column and row notation for elements of product spaces, according to convenience. For $\sigma, \sigma_{1}, \sigma_{2} \in \operatorname{Diff}^{+}\left(S^{1}\right)$, we abuse the norm notation and write $\|\sigma\|_{n}:=1+\|\dot{\sigma}\|_{n-1}$ and $\left\|\sigma_{1}-\sigma_{2}\right\|_{n}:=\sup \left\{\operatorname{dist}\left(\sigma_{1}(t), \sigma_{2}(t)\right) \mid t \in S^{1}\right\}+\left\|\dot{\sigma}_{1}-\dot{\sigma}_{2}\right\|_{n-1}$; similarly for $p, q \in \widetilde{\mathcal{M}}^{\text {int }}$ we write $\|p-q\|_{n}:=\sup \left\{\operatorname{dist}(p(t), q(t)) \mid t \in S^{1}\right\}+\|\dot{p}-\dot{q}\|_{n-1}$.

Observe that for any $q \in \widetilde{\mathcal{M}}_{\infty}^{\text {int }}$ and any $\sigma \in \operatorname{Diff}^{+}\left(S^{1}\right)$, we have

$$
\operatorname{dist}(q(\sigma(t)), q(t)) \leq\left|\int_{t}^{\sigma(t)}\right| \dot{q}(s)|d s| \leq \operatorname{dist}(t, \sigma(t))\|\dot{q}\|_{0},
$$

where in the integral we regard $t$ as an element of $[0,1)$ and $\sigma(t)$ as an element of $[t-1 / 2, t+1 / 2)$.

Since $p$ is now fixed, henceforth let us write

$$
q_{0}(f, a)=\tilde{\Phi}(f, a)=p+H_{p}(f, a) .
$$

Note that

$$
H_{p}(f, a)^{\cdot}=\left(\dot{f}+\dot{\theta}_{p} \hat{\lambda}_{p}(f, a)\right) \mathbf{N}_{p}-\left\langle f \dot{\theta}_{p}\right\rangle \mathbf{T}_{p}
$$

and that, using (4.11), for all $n \geq 0$ we have

$$
\left\|\dot{f}+\dot{\theta}_{p} \hat{\lambda}_{p}(f, a)\right\|_{n} \leq C\|(f, a)\|_{n+1}
$$

hence for all $n \geq 0$

$$
\left\|H_{p}(f, a)\right\|_{n} \leq C\|(f, a)\|_{n} .
$$

We also have

$$
q_{0}(f, a)^{\cdot}=\left(\dot{f}+\dot{\theta} \hat{\lambda}_{p}(f, a)\right) \mathbf{N}_{p}+\left(\ell_{p}-\left\langle f \dot{\theta}_{p}\right\rangle\right) \mathbf{T}_{p} .
$$

Assume henceforth that $\epsilon$ has been chosen such that for all $f \in U_{\infty}^{0, \epsilon}$ we have $\|f\|_{0}\left\|\dot{\theta}_{p}\right\|_{0}<\sqrt{2}-1$, and let $c_{f}=\ell_{p}-\left\langle f \dot{\theta}_{p}\right\rangle$. Since $\ell_{p} \geq \sqrt{2}$ for all $p \in \widetilde{\mathcal{M}}^{\text {int }}$, we then have

$$
1 \leq c_{f} \leq \ell_{q_{0}(f, a)} \leq\left\|q_{0}(f, a)^{*}\right\|_{0} \leq c_{f}+C\|(f, a)\|_{1} \leq \ell_{p}+C\|(f, a)\|_{1} .
$$

We therefore have

$$
\begin{aligned}
\left|q_{0}(f, a) \cdot(t)\right| & =c_{f}\left(1+\frac{\left(\dot{f}+\dot{\theta}_{p} \hat{\lambda}_{p}(f, a)\right)^{2}}{c_{f}^{2}}\right)^{1 / 2} \\
& =c_{f}\left(1+O\left(\|(f, a)\|_{1}^{2}\right)\right)
\end{aligned}
$$

which from (2.8) implies that $d\left(\bar{\sigma}_{q_{0}(f, a)}^{-1}(t), t\right)=O\left(\|(f, a)\|_{1}^{2}\right)$, hence that

$$
d\left(\sigma_{q_{0}(f, a)}^{-1}(t), t\right)=O\left(\|(f, a)\|_{1}^{2}\right) .
$$

(Here and below, $O(x)$ denotes any quantity bounded by a constant times $x$, where the constant may depend on $p$ but is otherwise universal; e.g. even if the quantity is $t$-dependent, the constant is not.) Therefore

$$
\sup _{t \in S^{1}}\left\{\operatorname{dist}\left(t, \sigma_{q_{0}(f, a)}(t)\right)\right\}=\sup _{t \in S^{1}}\left\{\operatorname{dist}\left(\sigma_{q_{0}(f, a)}^{-1}(t), t\right)\right\} \leq C\|(f, a)\|_{1}^{2} .
$$


Now let $q=\tilde{\Psi}_{p}(f, a, g)$, where $(f, a, g)$ lies in the domain indicated in (4.13). Combining (4.22) with (4.14), we have

$$
\sup _{t \in S^{1}}\left\{\operatorname{dist}\left(q\left(\sigma_{q_{0}(f, a)}(t)\right), q(t)\right)\right\} \leq C\|\dot{q}(s)\|_{0}\|(f, a)\|_{1}^{2} \leq C \epsilon^{2} .
$$

Therefore

$$
\begin{aligned}
\operatorname{dist}\left(\tilde{\Psi}_{p}(f, a, g)(t), p(t)\right)= & \left.\operatorname{dist}\left((p+Y)\left(\sigma_{q_{0}(f, a)}(t)\right), p(t)\right)\right\} \\
\leq & \operatorname{dist}\left((p+Y)\left(\sigma_{q_{0}(f, a)}(t)\right),(p+Y)(t)\right) \\
& +\operatorname{dist}((p+Y)(t), p(t)) \\
\leq & C \epsilon^{2}+\|Y\|_{0} \\
\leq & C \epsilon .
\end{aligned}
$$

Thus if we take $\epsilon$ sufficiently small, for each $(f, a, g) \in \operatorname{domain}\left(\tilde{\Psi}_{p}\right)$ there exists a unique $\mathbf{R}^{2}$-valued function $G(f, a, g) \in U_{\infty}^{0,1}$ such that $\tilde{\Psi}_{p}(f, a, g)=p+G(f, a, g)$; we can unambiguously write

$$
G(f, a, g)=\tilde{\Psi}_{p}(f, a, g)-p=\Theta_{p}^{-1}\left(\tilde{\Psi}_{p}(f, a, g)\right) .
$$

(Here and below, once we establish that something is true for $\epsilon$ sufficiently small, it is subsequently assumed that $\epsilon$ is that small.) Thus $G$ is an overlap map between $\left(\operatorname{Im}\left(\tilde{\Psi}_{p}\right), \tilde{\Psi}_{p}^{-1}\right)$, which we do not yet know is a smooth chart of $\widetilde{\mathcal{M}}_{\infty}^{\text {int }}$, and the chart $\left(\Theta_{p}\left(U_{j}^{0,1}\right), \Theta_{p}^{-1}\right)$ of $\widetilde{\mathcal{M}}_{\infty}^{\text {int }}$. We will see that if we shrink the domain of $\tilde{\Psi}_{p}$ sufficiently, $\left(\operatorname{Im}\left(\tilde{\Psi}_{p}\right), \tilde{\Psi}_{p}^{-1}\right)$ does become a smooth chart of $\widetilde{\mathcal{M}}_{\infty}^{\text {int }}$.

We claim that $G: C^{\infty}\left(S^{1}\right) \oplus \mathbf{R} \oplus C_{0}^{\infty}\left(S^{1}\right) \rightarrow C^{\infty}\left(S^{1}, \mathbf{R}^{2}\right) \cong C^{\infty}\left(S^{1}\right) \oplus C^{\infty}\left(S^{1}\right)$ is tamely smooth. To establish this, we write

$$
G(f, a, g)=G_{4}\left(G_{3}(f, a, g), G_{2}\left(G_{1}(f, a)\right)\right)
$$

where

$$
\begin{aligned}
G_{1}=\tilde{\Phi}_{p}:\left(\operatorname{dom}\left(\tilde{\Phi}_{p}\right) \subset C^{\infty}\left(S^{1}\right) \times \mathbf{R}\right) & \rightarrow \widetilde{\mathcal{M}}_{\infty}^{\text {int }}, \\
G_{2}: \widetilde{\mathcal{M}}_{\infty}^{\text {int }} & \rightarrow \operatorname{Diff}^{+}\left(S^{1}\right) \subset C^{\infty}\left(S^{1}, S^{1}\right), \\
G_{2}(q) & =\sigma_{q}, \\
G_{3}:\left(\operatorname{dom}\left(\tilde{\Psi}_{p}\right) \subset C^{\infty}\left(S^{1}\right) \times \mathbf{R} \times C_{0}^{\infty}\left(S^{1}\right)\right) & \rightarrow C^{\infty}\left(S^{1}, \mathbf{R}^{2}\right), \\
G_{3}(f, a, g) & =f \mathbf{N}_{p}+\left(\hat{\lambda}_{p}(f, a)+\rho(g)\right) \mathbf{T}_{p}, \\
G_{4}:\left(\operatorname{image}\left(\tilde{\Psi}_{p}\right) \subset C^{\infty}\left(S^{1}, \mathbf{R}^{2}\right)\right) \times \operatorname{Diff}^{+}\left(S^{1}\right) & \rightarrow C^{\infty}\left(S^{1}, \mathbf{R}^{2}\right), \\
G_{4}(Y, \sigma) & =(p+Y) \circ \sigma-p .
\end{aligned}
$$

From part (b), $\widetilde{\mathcal{M}}_{\infty}$ is a tame Fréchet manifold. From $[\underline{\mathrm{H}}$, Example I.4.1.3, p. 86, and Corollary II.2.3.2, p. 146, so is $C^{\infty}\left(S^{1}, S^{1}\right)$, of which $\operatorname{Diff}^{+}\left(S^{1}\right)$ is just an open subset; moreover its tangent space at any point is canonically isomorphic to $C^{\infty}\left(S^{1}\right)$, an identification that we will use implicitly below. Since the composition of tamely smooth maps is tamely smooth, it suffices to show that the $G_{i}$ are tamely smooth.

From (4.17) the map $H_{p}$ is tame, and since $H_{p}$ is linear it is therefore tamely smooth. Hence $\Theta_{p} \circ H_{p}=G_{1}$ is tamely smooth.

For $G_{2}$, multiple applications of the facts listed in Remark 5.1 yield first that the map $q \mapsto|\dot{q}|$ is tamely smooth, then that the map $q \mapsto \bar{\sigma}_{q}^{-1}$ (see (2.8)) is tamely smooth, then that the map $G_{5}: q \mapsto \sigma_{q}^{-1}$ is tamely smooth. Finally, we have 
$G_{2}=\operatorname{inv} \circ G_{5}$, where "inv" denotes inversion in the Fréchet Lie group $\operatorname{Diff}\left(S^{1}\right)$ (see $[\mathrm{H}$, p. 98, Definition I.4.6.1 and Example I.4.6.2), a tamely smooth map by $[\mathrm{H}$, Theorem II.2.3.5, p. 148. Therefore $G_{2}$ is tamely smooth.

$G_{3}$ is linear, and from (4.12) we have $\|\rho(g)\|_{j} \leq C\|g\|_{\max \{j-2,0\}}$ for $0 \leq j \leq \infty$. Combining this with (4.11) it follows that $G_{3}$ is tame, hence tamely smooth.

As for $G_{4}$, it follows from [H] Theorem II.2.3.3, p. 147, that $\Theta_{p}^{-1} \circ G_{4}:(Y, \sigma) \mapsto$ $(p+Y) \circ \sigma$ is a tamely smooth map, and hence so is $G_{4}$.

This establishes our claim that if we take $\epsilon$ sufficiently small, then $G$ is a tamely smooth map. Its derivative can be computed from (4.24) using the Chain Rule and the derivatives of the $G_{i}, 1 \leq i \leq 4$. The most complicated of these is $D G_{2}$, which we now compute; we leave computation of the others to the reader.

Let $F_{2}(q, t)=\sigma_{q}(t)$ and $F_{5}(q, t)=\sigma_{q}^{-1}(t)$. Differentiating the identity $F_{5}\left(q, F_{2}(q, t)\right)=t$ in the direction of $Z \in T_{q} \widetilde{\mathcal{M}}_{\infty}^{\text {int }}=C^{\infty}\left(S^{1}, \mathbf{R}^{2}\right)$ yields

$$
\left(\left.D G_{2}\right|_{q}\right)(Z)(t)=-\dot{\sigma}_{q}(t)\left(\left(\left.D G_{5}\right|_{q}(Z)\right) \circ \sigma_{q}\right)(t) .
$$

Let $\mathbf{T}_{q}(t)=\dot{q} /\|\dot{q}(t)\|$. From (2.8) $-(2.9)$ we compute

$$
\left.D G_{5}\right|_{q}(Z)=\frac{\hat{\alpha}_{q}(Z)}{\ell_{q}}
$$

where

$$
\begin{aligned}
\hat{\alpha}_{q}(Z) & =\bar{\alpha}_{q}(Z)-\left\langle\bar{\alpha}_{q}(Z)\right\rangle \quad \text { and } \\
\bar{\alpha}_{q}(Z)(t) & =\int_{0}^{t}\left(\mathbf{T}_{q}, \dot{Z}\right)(s) d s-\bar{\sigma}_{q}^{-1}(t) \int_{0}^{1}\left(\mathbf{T}_{q}, \dot{Z}\right)(s) d s
\end{aligned}
$$

(see (2.8) ). In this formula and others below, when $t$ appears as a limit of integration, we regard it as an element of $[0,1]$, and whenever $\bar{\sigma}_{q}^{-1}(t)$ appears as a multiplier of any real quantity, we regard $\bar{\sigma}_{q}^{-1}$ as a map $[0,1] \rightarrow[0,1]$ with $\bar{\sigma}_{q}(0)=0, \bar{\sigma}_{q}(1)=1$. Combining this with (4.25) gives

$$
\left(\left.D G_{2}\right|_{q}\right)(Z)(t)=-\frac{\hat{\alpha}_{q}(Z)\left(\sigma_{q}(t)\right)}{\left|\dot{q}\left(\sigma_{q}(t)\right)\right|} .
$$

Combining this result with the easy computation of $D G_{1}, D G_{3}$, and $D G_{4}$, we find that for $(f, a, g) \in \operatorname{dom}(G)$ and $\left(f_{1}, a_{1}, g_{1}\right) \in C^{\infty}\left(S^{1}\right) \times \mathbf{R} \times C_{0}^{\infty}\left(S^{1}\right)$ we have

$$
\left(\left.D G\right|_{(f, a, g)}\right)\left(\begin{array}{c}
f_{1} \\
a_{1} \\
g_{1}
\end{array}\right)=\left[Y_{1}-\alpha_{(p, f, a)}\left(f_{1}, a_{1}\right) \frac{(p+Y)^{\cdot}}{\left|q_{0}(f, a)^{\cdot}\right|}\right] \circ \sigma_{q_{0}(f, a)},
$$

where

$$
\begin{aligned}
Y_{1}=H_{p}\left(f_{1}, a_{1}\right)+\Lambda_{p}\left(g_{1}\right) & =f_{1} \mathbf{N}_{p}+\left(\hat{\lambda}_{p}\left(f_{1}, a_{1}\right)+\rho\left(g_{1}\right)\right) \mathbf{T}_{p}, \\
\alpha_{(p, f, a)}\left(f_{1}, a_{1}\right) & =\hat{\alpha}_{q_{0}(f, a)}\left(H_{p}\left(f_{1}, a_{1}\right)\right) \\
\text { and } \quad Y=G_{3}(f, a, g) & =f \mathbf{N}_{p}+\left(\hat{\lambda}_{p}(f, a)+\rho(g)\right) \mathbf{T}_{p} .
\end{aligned}
$$

We claim that for each $(f, a, g)$, the derivative $\left.D G\right|_{(f, a, g)}$ is an algebraic isomorphism. To establish this, write the right-hand side of (4.30) as $\left(\bar{\beta}_{1} \mathbf{N}_{p}+\bar{\beta}_{2} \mathbf{T}_{p}\right) \circ$ $\sigma_{q_{0}(f, a)}$, where $\bar{\beta}_{1}, \bar{\beta}_{2} \in C^{\infty}\left(S^{1}\right)$. We compute

$$
(p+Y)^{\cdot}=\left(\dot{f}+\left(\hat{\lambda}_{p}(f, a)+\rho(g)\right) \dot{\theta}_{p}\right) \mathbf{N}_{p}+\left(\ell_{p}-\left\langle f \dot{\theta}_{p}\right\rangle+\ell_{p}^{-1}\left(\omega_{g}-\left\langle\omega_{g}\right\rangle\right)\right) \mathbf{T}_{p}
$$


and therefore

$$
\begin{aligned}
& \bar{\beta}_{1}=f_{1}-\alpha_{(p, f, a)}\left(f_{1}, a_{1}\right) b_{1}(f, a, g), \\
& \bar{\beta}_{2}=\hat{\lambda}_{p}\left(f_{1}, a_{1}\right)+\rho\left(g_{1}\right)-\alpha_{(p, f, a)}\left(f_{1}, a_{1}\right) b_{2}(f, a, g),
\end{aligned}
$$

where

$$
\begin{aligned}
& b_{1}(f, a, g)=\frac{\dot{f}+\left(\hat{\lambda}_{p}(f, a)+\rho(g)\right) \dot{\theta}_{p}}{\left|q_{0}(f, a)\right|}, \\
& b_{2}(f, a, g)=\frac{\ell_{p}-\left\langle f \dot{\theta}_{p}\right\rangle+\ell_{p}^{-1}\left(\omega_{g}-\left\langle\omega_{g}\right\rangle\right)}{\left|q_{0}(f, a)^{\bullet}\right|} .
\end{aligned}
$$

Invertibility of $\left.D G\right|_{(f, a, g)}$ is equivalent to invertibility of the linear map

$$
\begin{aligned}
A_{(f, a, g)}: C^{\infty}\left(S^{1}\right) \oplus \mathbf{R} \oplus C_{0}^{\infty}\left(S^{1}\right) & \rightarrow C^{\infty}\left(S^{1}\right) \oplus C^{\infty}\left(S^{1}\right), \\
A_{(f, a, g)}\left(\begin{array}{c}
f_{1} \\
a_{1} \\
g_{1}
\end{array}\right) & =\left(\begin{array}{c}
\bar{\beta}_{1} \\
\bar{\beta}_{2}
\end{array}\right) .
\end{aligned}
$$

First consider $A_{0}:=A_{(0,0,0)}$. As the reader may check, $\alpha_{(p, 0,0)}$ is identically zero, so

$$
A_{0}\left(\begin{array}{c}
f_{1} \\
a_{1} \\
g_{1}
\end{array}\right)=\left(\begin{array}{c}
f_{1} \\
\hat{\lambda}_{p}\left(f_{1}, a_{1}\right)+\rho\left(g_{1}\right)
\end{array}\right) .
$$

The map $A_{0}$ is invertible; its inverse is given by

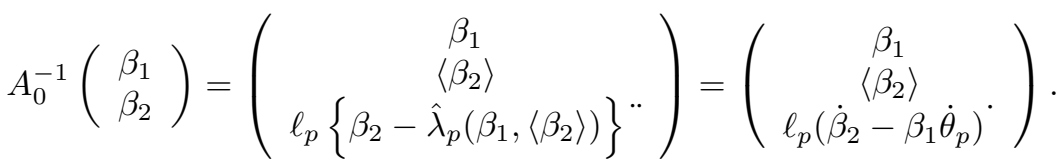

To examine invertibility of $A_{(f, a, g)}$ more generally, using (4.33)-4.34) we write $A_{(f, a, g)}=A_{0}-S=\left(I-S \circ A_{0}^{-1}\right) \circ A_{0}$, where

$$
S\left(\begin{array}{c}
f_{1} \\
a_{1} \\
g_{1}
\end{array}\right):=\alpha_{(p, f, a)}\left(f_{1}, a_{1}\right)\left(\begin{array}{c}
b_{1} \\
b_{2}
\end{array}\right) .
$$

(The operator $S$ and the functions $b_{1}, b_{2}$ depend on $(f, a, g)$, but to keep the notation from getting out of hand we will often suppress such parametric dependence. The same principle applies to other functions $b_{i}$ defined later.) Thus invertibility of $A_{(f, a, g)}$ is equivalent to invertibility of $I-S \circ A_{0}^{-1}$. Our strategy for showing invertibility of $I-S \circ A_{0}^{-1}$ will be as follows:

(1) show that, for $\epsilon$ in (4.13) sufficiently small, the formulas (4.39), (4.40) define an extension of $I-S A_{0}^{-1}$ to an invertible operator on $C^{1}\left(S^{1}\right) \oplus C^{1}\left(S^{1}\right)$; and

(2) show that the restriction of this inverse to $C^{\infty}\left(S^{1}\right) \oplus C^{\infty}\left(S^{1}\right)$ has range in $C^{\infty}\left(S^{1}\right) \oplus C^{\infty}\left(S^{1}\right)$.

Step 1. Let $\beta_{1}, \beta_{2} \in C^{\infty}\left(S^{1}\right)$. We will first need some estimates on $\alpha_{(p, f, a)}(\cdot, \cdot)$. Below, the notation $b_{i}$ is used for functions on $S^{1}$ that may depend on $p, f, a, g, f_{1}$, and $a_{1}$. After the introduction of one of these functions, we sometimes suppress the parameters it depends on (e.g. $b_{5}(f, a)$ may be written just $\left.b_{5}\right)$. 
Let $f_{1} \in C^{1}\left(S^{1}\right), a_{1} \in \mathbf{R}$, and to shorten the formulas below let $q_{0}=q_{0}(f, a)$. Each integrand on the right-hand side of (4.28) is

$$
\left|\dot{q}_{0}\right|^{-1}\left\{\left(\dot{f}+\dot{\theta}_{p} \hat{\lambda}_{p}(f, a)\right)\left(\dot{f}_{1}+\dot{\theta}_{p} \hat{\lambda}_{p}\left(f_{1}, a_{1}\right)\right)+\left(\left\langle f \dot{\theta}_{p}\right\rangle-\ell_{p}\right)\left\langle f_{1} \dot{\theta}_{p}\right\rangle\right\} .
$$

Hence

$$
\begin{aligned}
\alpha_{(p, f, a)}\left(f_{1}, a_{1}\right)(t)= & \int_{0}^{t} b_{3}\left(f, a ; f_{1}, a_{1}\right)(s) d s-\int_{0}^{1} \int_{0}^{t} b_{3}\left(f, a ; f_{1}, a_{1}\right)(s) d s d t \\
& -\left(\sigma_{q_{0}}^{-1}(t)-\frac{1}{2}\right)\left\langle b_{3}\left(f, a ; f_{1}, a_{1}\right)\right\rangle \\
& -\ell_{p}\left\langle f_{1} \dot{\theta}_{p}\right\rangle\left(b_{4}(f, a)(t)-\left\langle b_{4}(f, a)\right\rangle\right),
\end{aligned}
$$

where

$$
b_{3}\left(f, a ; f_{1}, a_{1}\right):=\frac{\left(\dot{f}+\dot{\theta}_{p} \hat{\lambda}_{p}(f, a)\right)\left(\dot{f}_{1}+\dot{\theta}_{p} \hat{\lambda}_{p}\left(f_{1}, a_{1}\right)\right)+\left\langle f \dot{\theta}_{p}\right\rangle\left\langle f_{1} \dot{\theta}_{p}\right\rangle}{\left|\dot{q}_{0}\right|}
$$

and

$$
b_{4}(f, a)(t):=\int_{0}^{t} \frac{1}{\left|\dot{q}_{0}(s)\right|} d s-\left\langle\frac{1}{\left|\dot{q}_{0}\right|}\right\rangle \sigma_{q_{0}}^{-1}(t) .
$$

Since $f_{1} \in C^{1}$, so is $\alpha_{(p, f, a)}\left(f_{1}, a_{1}\right)$, with $\alpha_{(p, f, a)}\left(f_{1}, a_{1}\right)^{\cdot}=b_{3}-\frac{\left\langle b_{3}\right\rangle}{\ell_{q_{0}}}\left|\dot{q}_{0}\right|-\ell_{p}\left\langle f_{1} \dot{\theta}_{p}\right\rangle \dot{b}_{4}$. From (4.22), $\left|\sigma_{q_{0}}^{-1}(t)-t\right| \leq C\|(f, a)\|_{1}^{2}$, implying that similarly $\left|\dot{b}_{4}(t)\right| \leq C\|(f, a)\|_{1}$. From (4.43) we have $\left\|b_{3}\right\|_{0} \leq C\|(f, a)\|_{1}\left\|\left(f_{1}, a_{1}\right)\right\|_{1}$. Since $\left|\sigma_{q_{0}}^{-1}(t)\right|$ and $\ell_{q_{0}}^{-1}\left\|\dot{q}_{0}(t)\right\|$ are $\leq C$, it follows that

$$
\left\|\alpha_{(p, f, a)}\left(f_{1}, a_{1}\right)\right\|_{1} \leq C\|(f, a)\|_{1}\left\|\left(f_{1}, a_{1}\right)\right\|_{1} .
$$

Next, from (4.38) and (4.33)-(4.34) we compute

$$
S A_{0}^{-1}\left(\begin{array}{c}
\beta_{1} \\
\beta_{2}
\end{array}\right)=\alpha_{(p, f, a)}\left(\beta_{1},\left\langle\beta_{2}\right\rangle\right)\left(\begin{array}{c}
b_{1} \\
b_{2}
\end{array}\right) .
$$

Since $\beta_{1} \in C^{1}$ we have $\alpha_{(p, f, a)}\left(\beta_{1},\left\langle\beta_{2}\right\rangle\right) \in C^{1}$; since $b_{i} \in C^{\infty}$ it follows that $S A_{0}^{-1}\left(\begin{array}{c}\beta_{1} \\ \beta_{2}\end{array}\right) \in C^{1}$. From (4.35),

$$
\begin{aligned}
\left\|b_{1}\right\|_{1} & \leq C\left(\|\dot{f}\|_{1}+\left\|\hat{\lambda}_{p}(f, a)\right\|_{1}+\|\rho(g)\|_{1}\right) \cdot\left\|\frac{1}{\left|\dot{q}_{0}\right|}\right\|_{1} \\
& \leq C\left(\|(f, a)\|_{2}+\|g\|_{0}\right) \cdot C \\
& \leq C\|(f, a, g)\|_{2} .
\end{aligned}
$$

Similarly $\left\|b_{2}\right\|_{1} \leq C\left(1+\|(f, a, g)\|_{2}\right) \leq C$. Hence $\left\|\left(b_{1}, b_{2}\right)\right\|_{1} \leq C$, so

$$
\left\|\alpha_{(p, f, a)}\left(\beta_{1},\left\langle\beta_{2}\right\rangle\right)\left(\begin{array}{c}
b_{1} \\
b_{2}
\end{array}\right)\right\|_{1} \leq C\|(f, a)\|_{1}\left\|\left(\begin{array}{c}
\beta_{1} \\
\beta_{2}
\end{array}\right)\right\|_{1} .
$$

It follows that $S A_{0}^{-1}$ extends to a bounded operator $C^{1}\left(S^{1}\right) \oplus C^{1}\left(S^{1}\right) \rightarrow C^{1}\left(S^{1}\right) \oplus$ $C^{1}\left(S^{1}\right)$, with

$$
\left\|S A_{0}^{-1}\right\|_{\mathrm{op}, 1} \leq C\|(f, a)\|_{1},
$$

where $\|\cdot\|_{\mathrm{op}, j}$ denotes the operator norm on $\operatorname{Hom}\left(C^{j}\left(S^{1}\right) \oplus C^{j}\left(S^{1}\right)\right)$. Thus if we take $\epsilon$ sufficiently small in (4.13), we have $\left\|S A_{0}^{-1}\right\|_{\mathrm{op}, 1}<1$. Hence $I-S A_{0}^{-1}$ is invertible on $C^{1}\left(S^{1}\right) \oplus C^{1}\left(S^{1}\right)$, with its inverse given by the norm-convergent Neumann series $I+\sum_{n=1}^{\infty}\left(S A_{0}^{-1}\right)^{n}$. This completes Step 1 . 
Step 2. Define the function $b_{6}\left(f, a ; f_{1}, a_{1}\right): S^{1} \rightarrow \mathbf{R}$ by writing

$$
\begin{aligned}
b_{3}\left(f, a ; f_{1}, a_{1}\right) & =b_{5}(f, a) \dot{f}_{1}+b_{6}\left(f, a ; f_{1}, a_{1}\right), \\
\text { where } \quad b_{5}(f, a) & =\frac{\dot{f}+\dot{\theta}_{p} \hat{\lambda}_{p}(f, a)}{\left|\dot{q}_{0}\right|} .
\end{aligned}
$$

Integrating by parts the term $b_{5}(f, a) \dot{f}_{1}$ in $b_{3}\left(f, a ; f_{1}, a_{1}\right)$, we have

$$
\int_{0}^{t} b_{3}(s) d s=b_{5}(t) f_{1}(t)-b_{5}(0) f_{1}(0)-\int_{0}^{t} \dot{b}_{5}(s) f_{1}(s) d s+\int_{0}^{t} b_{6}(s) d s
$$

and

$$
\left\langle b_{3}\right\rangle=\left\langle-\dot{b}_{5} f_{1}+b_{6}\right\rangle
$$

We have $\left\|b_{5}\right\|_{0} \leq C\|(f, a)\|_{1}$ and $\left\|b_{5}\right\|_{1} \leq C\|(f, a)\|_{2}$, while $\left\|b_{6}\right\|_{0} \leq$ $C\|(f, a)\|_{1}\left\|\left(f_{1}, a_{1}\right)\right\|_{0}$. Hence

$$
\left|\left\langle b_{3}\right\rangle\right| \leq C\left(\left\|b_{5}\right\|_{1}\left\|f_{1}\right\|_{0}+\left\|b_{6}\right\|_{0}\right) \leq C\|(f, a)\|_{2}\left\|\left(f_{1}, a_{1}\right)\right\|_{0} .
$$

Substituting (4.47) and (4.48) into (4.42), we have

$$
\alpha_{(p, f, a)}\left(f_{1}, a_{1}\right)(t)=b_{5}(t) f_{1}(t)-\left\langle b_{5} f_{1}\right\rangle+b_{7}\left(f, a ; f_{1}, a_{1}\right)(t)-\left\langle b_{7}\left(f, a ; f_{1}, a_{1}\right)\right\rangle
$$

where

$$
b_{7}\left(f, a ; f_{1}, a_{1}\right)(t)=\int_{0}^{t}\left(-\dot{b}_{5} f_{1}+b_{6}\right)(s) d s-\sigma_{q_{0}}^{-1}(t)\left\langle b_{3}\right\rangle-\ell_{p}\left\langle f_{1} \dot{\theta}_{p}\right\rangle b_{4}(t),
$$

implying

$$
\left\|\alpha_{(p, f, a)}\left(f_{1}, a_{1}\right)\right\|_{0} \leq C\|(f, a)\|_{2}\left\|\left(f_{1}, a_{1}\right)\right\|_{0} .
$$

Now suppose $\beta_{3}, \beta_{4} \in C^{\infty}\left(S^{1}\right)$ and let $\left(\begin{array}{c}\beta_{1} \\ \beta_{2}\end{array}\right)=\left(I-S A_{0}^{-1}\right)^{-1}\left(\begin{array}{c}\beta_{3} \\ \beta_{4}\end{array}\right)$. Initially, from Step 1 we know only that $\beta_{1}, \beta_{2} \in C^{1}$. However, from $\left(I-S A_{0}^{-1}\right)\left(\begin{array}{c}\beta_{1} \\ \beta_{2}\end{array}\right)$ $=\left(\begin{array}{c}\beta_{3} \\ \beta_{4}\end{array}\right)$ we have

$$
\begin{aligned}
& \beta_{1}=\beta_{3}+\left(b_{5} \beta_{1}+b_{7}\left(f, a ; \beta_{1},\left\langle\beta_{2}\right\rangle\right)\right) b_{1}, \\
& \beta_{2}=\beta_{4}+\left(b_{5} \beta_{1}+b_{7}\left(f, a ; \beta_{1},\left\langle\beta_{2}\right\rangle\right)\right) b_{2} .
\end{aligned}
$$

Similarly to (4.45) we have $\left\|b_{1}\right\|_{0} \leq C\|(f, a, g)\|_{1}$, and we have already bounded $\left\|b_{5}\right\|_{0}$ by $C\|(f, a)\|_{1}$. Hence by choosing $\epsilon$ in (4.13) small enough we can ensure that $\left\|b_{1} b_{5}\right\|_{0} \leq 1 / 2$, implying

$$
\begin{aligned}
\beta_{1} & =\frac{\beta_{3}+b_{1} b_{7}\left(f, a ; \beta_{1},\left\langle\beta_{2}\right\rangle\right)}{1-b_{1} b_{5}} \\
\beta_{2} & =\beta_{4}+\frac{b_{2}}{1-b_{1} b_{5}}\left(b_{5} \beta_{3}+b_{7}\left(f, a ; \beta_{1},\left\langle\beta_{2}\right\rangle\right)\right) .
\end{aligned}
$$

From (4.53), tracing the definition of $b_{7}$ back through (4.50), (4.47), and (4.43), we see that for $j \geq 1$, if $\beta_{1} \in C^{j}$ then $b_{7}\left(f, a ; \beta_{1},\left\langle\beta_{2}\right\rangle\right) \in C^{j+1}$. Since $\beta_{3}, b_{1}$, and $b_{5}$ are all $C^{\infty}$, it then follows that the right-hand side of (4.53) is in $C^{j+1}$; hence $\beta_{1} \in C^{j+1}$. Since $\beta_{1} \in C^{1}$, it follows by induction that $\beta_{1} \in C^{\infty}$ and $b_{7}\left(f, a ; \beta_{1},\left\langle\beta_{2}\right\rangle\right) \in C^{\infty}$. It then follows from (4.52) that $\beta_{2} \in C^{\infty}$. This completes Step 2.

Since invertibility of $A_{(f, a, g)}$ implies invertibility of $\left.D G\right|_{(f, a, g)}$, we have now established that there exists an open neighborhood $U$ of $(0,0,0) \in C^{\infty}\left(S^{1}\right) \oplus \mathbf{R} \oplus$ 
$C_{0}^{\infty}\left(S^{1}\right)$ on which $G$ is tamely smooth, and such that for all $(f, a, g) \in U$, the derivative $\left.D G\right|_{(f, a, g)}$ is invertible. We claim next that the map

$$
\begin{aligned}
U \times C^{\infty}\left(S^{1}, \mathbf{R}^{2}\right) & \rightarrow C^{\infty}\left(S^{1}\right) \oplus \mathbf{R} \oplus C_{0}^{\infty}\left(S^{1}\right), \\
((f, a, g), X) & \mapsto\left(\left.D G\right|_{(f, a, g)}\right)^{-1}(X),
\end{aligned}
$$

is continuous and tame.

For this, first consider the map

$$
\begin{aligned}
K: U \times\left(C^{\infty}\left(S^{1}\right) \oplus C^{\infty}\left(S^{1}\right)\right) & \rightarrow C^{\infty}\left(S^{1}\right) \oplus C^{\infty}\left(S^{1}\right), \\
\left((f, a, g),\left(\begin{array}{c}
\beta_{3} \\
\beta_{4}
\end{array}\right)\right) & \mapsto\left(I-S_{(f, a, g)} A_{0}^{-1}\right)^{-1}\left(\begin{array}{c}
\beta_{3} \\
\beta_{4}
\end{array}\right) .
\end{aligned}
$$

We claim that $K$ is tame and continuous. For both parts of this assertion, we need to examine for all finite $n$ the behavior of the right-hand side of (4.44) in the $C^{n}$ topology as the quintuple $\left(f, a, g, \beta_{3}, \beta_{4}\right)$ varies. We have already bounded the functions $b_{i}$ in various $n$-norms for small $n$. For larger $n$, we can bound the norms in a similar fashion, making liberal use of the restriction $\|(f, a)\|_{n} \leq\|(f, a, g)\|_{n}<$ $\epsilon<1$ for $n \leq 2$. To obtain tame estimates we will need to use the interpolation inequality

$$
\|h k\|_{n} \leq C(n)\left(\|h\|_{n}\|k\|_{0}+\|h\|_{0}\|k\|_{n}\right)
$$

([H] , Corollary II.2.2.3, p. 144). The estimates of $\left\|b_{i}\right\|_{n}$ require estimates on the reciprocals of some functions; for these we use Lemma 4.4. which we state and prove after completing the current proof. We spare the reader the tedious details and simply list the results in an order in which they are easily derived:

$$
\begin{aligned}
\left\|b_{4}(f, a)\right\|_{n} & \leq C(n)\|(f, a)\|_{\max \{n, 1\}}, \\
\left\|b_{3}\left(f, a ; f_{1}, a_{1}\right)\right\|_{n} & \leq C(n)\|(f, a)\|_{n+1}\left\|\left(f_{1}, a_{1}\right)\right\|_{n+1}, \\
\left\|\alpha_{(p, f, a)}\left(f_{1}, a_{1}\right)\right\|_{n} & \leq C(n)\|(f, a)\|_{\max \{n, 2\}}\left\|\left(f_{1}, a_{1}\right)\right\|_{n}, \\
\left\|b_{5}(f, a)\right\|_{n} & \leq C(n)\|(f, a)\|_{n+1}, \\
\left\|b_{7}\left(f, a ; f_{1}, a_{1}\right)\right\|_{n} & \leq C(n)\|(f, a)\|_{\max \{n+1,2\}}\left\|\left(f_{1}, a_{1}\right)\right\|_{0}, \\
\left\|b_{i}(f, a, g)\right\|_{n} & \leq C(n)\|(f, a, g)\|_{n+1}, i=1,2, \text { and }
\end{aligned}
$$

where $C(n)$ denotes a constant that depends only on $p$ and $n$. Using these bounds, from (4.53)-(4.54) we deduce that

$$
\left\|\left(\beta_{1},\left\langle\beta_{2}\right\rangle\right)\right\|_{0} \leq\left\|\left(\beta_{1}, \beta_{2}\right)\right\|_{0} \leq C\left(\left\|\left(\beta_{3}, \beta_{4}\right)\right\|_{0}+\epsilon^{2}\left\|\left(\beta_{1},\left\langle\beta_{2}\right\rangle\right)\right\|_{0}\right)
$$

and hence, after shrinking $\epsilon$, that

$$
\left\|\left(\beta_{1},\left\langle\beta_{2}\right\rangle\right)\right\|_{0} \leq\left\|\left(\beta_{1}, \beta_{2}\right)\right\|_{0} \leq C\left\|\left(\beta_{3}, \beta_{4}\right)\right\|_{0} .
$$

Substituting $\left(f_{1}, a_{1}\right)=\left(\beta_{1},\left\langle\beta_{2}\right\rangle\right)$ into the bounds in the list above, we then use (4.53) - 4.54) again to find that, for all $n \geq 1$,

$$
\left\|\left(\beta_{1}, \beta_{2}\right)\right\|_{n} \leq C(n)\left(\epsilon^{2}+\left\|\left(\beta_{3}, \beta_{4}\right)\right\|_{n}+\epsilon^{2}\left\|\left(\beta_{3}, \beta_{4}\right)\right\|_{0}\|(f, a, g)\|_{n+1}\right) .
$$

Thus, if $B$ is a ball in $C^{\infty}\left(S^{1}\right) \oplus C^{\infty}\left(S^{1}\right)$ of finite $C^{0}$-radius, then on $U \times B$ we have $\left\|\left(\beta_{3}, \beta_{4}\right)\right\|_{0} \leq C(B)$, implying

$$
\left\|\left(\beta_{1}, \beta_{2}\right)\right\|_{n} \leq C(n, B)\left(1+\|(f, a, g)\|_{n+1}+\left\|\left(\beta_{3}, \beta_{4}\right)\right\|_{n}\right) .
$$

Hence $K$ is tame. 
For continuity at a point $p=\left((f, a, g),\left(\beta_{3}, \beta_{4}\right)\right)$, it suffices to check that for all $n$ and all $\delta>0$, there exist $\delta^{\prime}>0$ and $n^{\prime}$ such that $K$ maps the $n^{\prime}$-norm ball of radius $\delta^{\prime}$ centered at $p$ into the $n$-norm ball of radius $\delta$ centered at $K(p)$; equivalently, that for all $n$ there exists $n^{\prime}$ such that $K$ is continuous at $p$ as a map from $U \times B$ with the $n^{\prime}$-norm topology to $C^{\infty}\left(S^{1}\right) \oplus C^{\infty}\left(S^{1}\right)$ with the $n$-norm topology.

Holding $(f, a, g)$ fixed, let $P=\left(I-S A_{0}^{-1}\right)^{-1}$. We can then rewrite (4.61) as

$$
\left\|P\left(\begin{array}{c}
\beta_{3} \\
\beta_{4}
\end{array}\right)\right\|_{n} \leq C(n, f, a, g)\left(\epsilon^{2}+\left\|\left(\begin{array}{c}
\beta_{3} \\
\beta_{4}
\end{array}\right)\right\|_{n}\right) .
$$

Let $B_{\text {in }}$ denote the ball $\left\{\left(\beta_{3}, \beta_{4}\right) \in C^{\infty}\left(S^{1}\right) \oplus C^{\infty}\left(S^{1}\right) \mid\left\|\left(\beta_{3}, \beta_{4}\right)\right\|_{n}<\epsilon^{2}\right\}$ and let $B_{\text {out }}$ denote its complement. Then on $B_{\text {out }}$ we have

$$
\left\|P\left(\begin{array}{l}
\beta_{3} \\
\beta_{4}
\end{array}\right)\right\|_{n} \leq C(n, f, a, g)\left\|\left(\begin{array}{c}
\beta_{3} \\
\beta_{4}
\end{array}\right)\right\|_{n} .
$$

However, every element of $B_{\text {in }}$ in proportional to an element of $B_{\text {out }}$, and $P$ is linear; hence (4.62) holds for all $\left(\beta_{3}, \beta_{4}\right) \in C^{\infty}\left(S^{1}\right) \oplus C^{\infty}\left(S^{1}\right)$, and $P$ is bounded in the (op, $n)$-norm.

Let $p^{\prime}=\left(\left(f^{\prime}, a^{\prime}, g^{\prime}\right),\left(\beta_{3}^{\prime}, \beta_{4}^{\prime}\right)\right) \in U \times\left(C^{\infty}\left(S^{1}\right) \oplus C^{\infty}\left(S^{1}\right)\right)$. From (4.49) and the definitions of $b_{5}$ and $b_{7}$, and it is straightforward (if tedious) to verify that for $n \geq 1$,

$$
\begin{aligned}
& \left\|\alpha_{\left(p, f^{\prime}, a^{\prime}\right)}\left(f_{1}, a_{1}\right)-\alpha_{(p, f, a)}\left(f_{1}, a_{1}\right)\right\|_{n} \\
& \quad \leq C(f, a, n)\left\|\left(\begin{array}{c}
f^{\prime} \\
a^{\prime}
\end{array}\right)-\left(\begin{array}{c}
f \\
a
\end{array}\right)\right\|_{n+1}\left\|\left(\begin{array}{c}
f_{1} \\
a_{1}
\end{array}\right)\right\|_{n} .
\end{aligned}
$$

for $\left(f^{\prime}, a^{\prime}\right)$ sufficiently close to $(f, a)$ in the $(n+1)$-norm. Similarly, from (4.35)(4.36) we find, for $n \geq 1$, that

$$
\left\|\left(\begin{array}{c}
b_{1}\left(f^{\prime}, a^{\prime}, g^{\prime}\right) \\
b_{2}\left(f^{\prime}, a^{\prime}, g^{\prime}\right)
\end{array}\right)-\left(\begin{array}{c}
b_{1}(f, a, g) \\
b_{2}(f, a, g)
\end{array}\right)\right\|_{n} \leq C(f, a, g, n)\left\|\left(\begin{array}{c}
f^{\prime} \\
a^{\prime} \\
g^{\prime}
\end{array}\right)-\left(\begin{array}{c}
f \\
a \\
g
\end{array}\right)\right\|_{n+1}
$$

for $\left(f^{\prime}, a^{\prime}, g^{\prime}\right)$ sufficiently close to $(f, a, g)$ in the $(n+1)$-norm. Hence, using (4.44) and writing $S^{\prime}=S_{\left(f^{\prime}, a^{\prime}, g^{\prime}\right)}, S=S_{(f, a, g)}$, we have

$$
\left\|\left(S^{\prime} A_{0}^{-1}-S A_{0}^{-1}\right)\left(\begin{array}{c}
\beta_{1} \\
\beta_{2}
\end{array}\right)\right\|_{n} \leq C(f, a, g, n)\left\|\left(\begin{array}{c}
f^{\prime} \\
a^{\prime} \\
g^{\prime}
\end{array}\right)-\left(\begin{array}{c}
f \\
a \\
g
\end{array}\right)\right\|_{n+1}\left\|\left(\begin{array}{c}
\beta_{1} \\
\beta_{2}
\end{array}\right)\right\|_{n}
$$

for all $n \geq 1$. Thus, given $n$, there exists $\delta>0$ such that for all $\left(f^{\prime}, a^{\prime}, g^{\prime}\right)$ in the $(n+1)$-norm $\delta$-ball centered at $(f, a, g),\left\|\left(S^{\prime} A_{0}^{-1}-S A_{0}^{-1}\right)\right\|_{\mathrm{op}, n}$ is as small as we like. Hence the map $U \rightarrow \operatorname{Hom}\left(C^{\infty}\left(S^{1}\right) \oplus C^{\infty}\left(S^{1}\right)\right),(f, a, g) \mapsto I-S_{(f, a, g)} A_{0}^{-1}$, is continuous with respect to the $(n+1)$-norm topology on $U$ and the (op, $n)$ norm topology on $\operatorname{Hom}\left(C^{\infty}\left(S^{1}\right) \oplus C^{\infty}\left(S^{1}\right)\right)$, and extends to a continuous map $\left(U,\|\|_{n+1}\right) \rightarrow\left(\operatorname{Hom}\left(C^{n}\left(S^{1}\right) \oplus C^{n}\left(S^{1}\right)\right)\right.$, \|\|$\left._{\mathrm{op}, n}\right)$ with image in the space of invertible operators, $\operatorname{Aut}\left(C^{n}\left(S^{1}\right) \oplus C^{n}\left(S^{1}\right)\right)$. Since for any Banach space $E$, inversion on $\operatorname{Aut}(E)$ is continuous in the operator-norm topology, and since (as we have seen) for $(f, a, g) \in U,\left(I-S_{(f, a, g)} A_{0}^{-1}\right)^{-1}$ preserves $C^{\infty}\left(S^{1}\right) \oplus C^{\infty}\left(S^{1}\right)$, it follows that the map

$$
(f, a, g) \mapsto\left(I-S_{(f, a, g)} A_{0}^{-1}\right)^{-1}
$$

is continuous as a map $\left(U,\|\|_{n+1}\right) \rightarrow\left(\operatorname{Hom}\left(C^{\infty}\left(S^{1}\right) \oplus C^{\infty}\left(S^{1}\right)\right)\right.$, \|\|$\left._{\mathrm{op}, n}\right)$. 
Let $p=\left((f, a, g),\left(\beta_{3}, \beta_{4}\right)\right), p^{\prime}=\left(\left(f^{\prime}, a^{\prime}, g^{\prime}\right),\left(\beta_{3}^{\prime}, \beta_{4}^{\prime}\right)\right) \in U \times\left(C^{\infty}\left(S^{1}\right) \oplus C^{\infty}\left(S^{1}\right)\right)$ and let $P=\left(I-S_{(f, a, g)} A_{0}^{-1}\right)^{-1}, P^{\prime}=\left(I-S_{\left(f^{\prime} a^{\prime} g^{\prime}\right)} A_{0}^{-1}\right)^{-1}$. Then

$$
K\left(p^{\prime}\right)-K(p)=\left(P^{\prime}-P\right)\left(\begin{array}{c}
\beta_{3}^{\prime} \\
\beta_{4}^{\prime}
\end{array}\right)+P\left(\left(\begin{array}{c}
\beta_{3}^{\prime} \\
\beta_{4}^{\prime}
\end{array}\right)-\left(\begin{array}{c}
\beta_{3} \\
\beta_{4}
\end{array}\right)\right) .
$$

From this and the continuity of (4.63) mentioned above, it follows easily that $K$ is continuous as a map

$$
\left(U \times\left(C^{\infty}\left(S^{1}\right) \oplus C^{\infty}\left(S^{1}\right)\right),\|\|_{n+1}\right) \rightarrow\left(C^{\infty}\left(S^{1}\right) \oplus C^{\infty}\left(S^{1}\right),\|\|_{n}\right) .
$$

Since this is true for all $n, K$ is continuous with respect to the Fréchet topologies on source and target.

Thus as claimed, $K$ is continuous and tame. From (4.39) it is easily seen that $A_{0}^{-1}$ is also continuous and tame, and hence so is $A_{0}^{-1} \circ K$. From (4.37), (4.33)(4.34), and (4.57)-(4.59), the map

$$
\left((f, a, g),\left(f_{1}, a_{1}, g_{1}\right)\right) \mapsto A_{(f, a, g)}\left(\begin{array}{c}
f_{1} \\
a_{1} \\
g_{1}
\end{array}\right)
$$

is easily seen to be continuous and tame. We have already seen that the maps $G_{5}: q \mapsto \sigma_{q}^{-1}$ and $G_{1}$ are tamely smooth. The linear map

$$
\begin{aligned}
\iota: C^{\infty}\left(S^{1}\right) \oplus C^{\infty}\left(S^{1}\right) & \rightarrow C^{\infty}\left(S^{1}, \mathbf{R}^{2}\right) \\
\left(\beta_{1}, \beta_{2}\right) & \mapsto \beta_{1} \mathbf{N}_{p}+\beta_{2} \mathbf{T}_{p}
\end{aligned}
$$

and its inverse are easily seen to be tame (hence continuous). Define $G_{6}: U \times$ $C^{\infty}\left(S^{1}, \mathbf{R}^{2}\right) \rightarrow C^{\infty}\left(S^{1}, \mathbf{R}^{2}\right)$ by $G_{6}((f, a, g), Y)=Y \circ G_{2} \circ G_{1}(f, a, g)$. Then $G_{6}$ is continuous and tame, and from (4.30) we have

$$
\left.D G\right|_{(f, a, g)}\left(\begin{array}{c}
f_{1} \\
a_{1} \\
g_{1}
\end{array}\right)=G_{6}\left((f, a, g),\left[\left(\iota \circ A_{(f, a, g)}\right)\left(\begin{array}{c}
f_{1} \\
a_{1} \\
g_{1}
\end{array}\right)\right]\right) .
$$

Hence on $U$, where we have established that $A_{(f, a, g)}^{-1}$ exists, $\left(\left.D G\right|_{(f, a, g)}\right)^{-1}$ exists and is given by

$\left(\left.D G\right|_{(f, a, g)}\right)^{-1}(X)=\left(\iota \circ A_{(f, a, g)}\right)^{-1}\left(X \circ \sigma_{q_{0}(f, a)}^{-1}\right)=\left(A_{(f, a, g)}^{-1} \circ \iota^{-1} \circ G_{6}\right)((f, a, g), X)$.

Thus the map (4.55) is continuous and tame. Using the last item listed in Remark 5.1, since $G$ is tamely smooth, so is (4.55). Thus Theorem 5.2 implies that there exist open neighborhoods $W_{1}(p), W_{2}(p)$ of the origins in $C^{\infty}\left(S^{1}\right) \oplus \mathbf{R} \oplus$ $C_{0}^{\infty}\left(S^{1}\right), C^{\infty}\left(S^{1}, \mathbf{R}^{2}\right)$ respectively, such that $G: W_{1}(p) \rightarrow W_{2}(p)$ is invertible, with $G^{-1}$ a tamely smooth map. Let $U(p)=\tilde{\Psi}_{p}\left(W_{1}(p)\right)$. Since $G=\Theta_{p}^{-1} \circ \tilde{\Psi}_{p}$ (see (4.23) $)$, it follows that $\left(U(p), \tilde{\Psi}_{p}^{-1}\right)$ is compatible with the chart $\left(\Theta_{p}\left(U_{j}^{0,1}\right), \Theta_{p}^{-1}\right)$

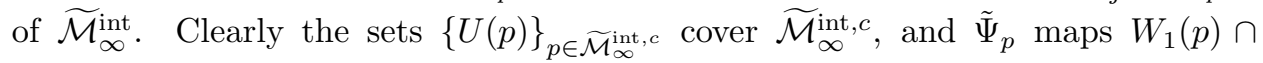
$\left(C^{\infty}\left(S^{1}\right) \oplus \mathbf{R}\right) \times\{0\}$ into $\widetilde{\mathcal{M}}_{\infty}^{\text {int }, c}$. Thus the collection $\left\{U(p), \tilde{\Psi}_{p}^{-1}\right\}_{p \in \widetilde{\mathcal{M}}_{\infty}^{\text {int }, c}}$ is a tame submanifold atlas for $\widetilde{\mathcal{M}}_{\infty}^{\text {int }, c} \subset \widetilde{\mathcal{M}}_{\infty}^{\text {int }}$, and $\widetilde{\mathcal{M}}_{\infty}^{\text {int }, c}$ is a tame Fréchet manifold canonically modeled on $C^{\infty}\left(S^{1}\right) \oplus \mathbf{R}$. 
(e) Let $\Sigma \in \mathcal{M}_{j}^{\text {int }}$, let $p \in \widetilde{\mathcal{M}}_{j}^{\text {int }}$ be a parametrization of $\Sigma$, and let $V$ be a $C^{j}$ pseudo-tangent vector field along $p$ as in (c); thus $J V$ is transverse to $p$. Define

$$
\begin{aligned}
E_{p, V}: S^{1} \times \mathbf{R} & \rightarrow S^{1} \times S^{1}, \\
E_{p, V}(t, s) & =p(t)+s \mathrm{~J} V(t) ;
\end{aligned}
$$

for $\epsilon$ sufficiently small, $\left.E_{p, V}\right|_{S^{1} \times(-\epsilon, \epsilon)}$ is a $C^{j}$ diffeomorphism onto its image.

Every sufficiently $C^{1}$-small $C^{j}$ perturbation of $p$ lies in $\widetilde{\mathcal{M}}_{j}^{\text {int }}$, so for $\epsilon>0$ sufficiently small we can define a $C^{\infty}$ map

$$
\begin{aligned}
\tilde{\Phi}_{p, V}:\left\{B_{\epsilon}(0) \subset C^{j}\left(S^{1}\right)\right\} & \rightarrow \widetilde{\mathcal{M}}_{j}^{\text {int }}, \\
f & \mapsto p+f \mathrm{~J} V .
\end{aligned}
$$

We will show that maps of the form $\Phi_{p, V}:=\pi_{\widetilde{\mathcal{M}} \rightarrow \mathcal{M}} \circ \tilde{\Phi}_{p, V}$ provide a $C^{0}$ atlas for $\mathcal{M}_{j}^{\text {int }}$. Clearly the domains of these maps cover $\mathcal{M}_{j}^{\text {int }}$; we need only examine the overlap maps on intersections of images of these maps.

Note that $\left(\tilde{\Phi}_{p, V}(f)\right)(t)=E_{p, V}(t, f(t))$. Given pseudo-tangent vector fields $V_{i}$ along $p_{i}, i=1,2$, and maps $f_{i} \in \operatorname{domain}\left(\Phi_{p_{i}, V_{i}}\right)$, we have $\left.\Phi_{p_{2}, V_{2}}\left(f_{2}\right)=\Phi_{p_{1}, V_{1}}\left(f_{1}\right)\right)$ iff there exists $\sigma \in \operatorname{Diff}_{j}^{+}\left(S^{1}\right)$ such that $\tilde{\Phi}_{p_{2}, V_{2}}\left(f_{2}\right) \circ \sigma=\tilde{\Phi}_{p_{1}, V_{1}}\left(f_{1}\right)$, i.e. iff

$$
\left(\sigma(t), f_{2}(\sigma(t))\right)=E_{p_{2}, V_{2}}^{-1} \circ E_{p_{1}, V_{1}}\left(t, f_{1}(t)\right) \quad \forall t \in S^{1} .
$$

If we take $p_{2}=p_{1}$ and $V_{2}=V_{1}$, (4.65) implies that $\sigma(t) \equiv t$ and hence that $f_{2}=f_{1}$; thus for each $V$ the map $\pi_{\widetilde{\mathcal{M}} \rightarrow \mathcal{M}} \circ \tilde{\Phi}_{p, V}$ is injective. For more general $p_{i}, V_{i}$, (4.65) implies that

$$
\sigma=A\left(f_{1}\right), \quad f_{2}=B\left(f_{1}, \sigma\right)=G_{7}\left(f_{1}\right) \circ \sigma^{-1},
$$

where $A(f)=\operatorname{proj}_{1} \circ E_{p_{2}, V_{2}}^{-1} \circ E_{p_{1}, V_{1}} \circ(\mathrm{id}, f), G_{7}(f)=\operatorname{proj}_{2} \circ E_{p_{2}, V_{2}}^{-1} \circ E_{p_{1}, V_{1}} \circ(\mathrm{id}, f)$, and where $\operatorname{proj}_{1}$, $\operatorname{proj}_{2}$ are the projections onto the first and second factors of $S^{1} \times \mathbf{R}$. Thus $f_{2}=\left(\Phi_{p_{2}, V_{2}^{-1}} \circ \Phi_{p_{1}, V_{1}}\right)\left(f_{1}\right)=B\left(f_{1}, A\left(f_{1}\right)^{-1}\right)$, and it is easily seen that $A$ and $B$ are continuous; hence $\Phi_{p_{2}, V_{2}^{-1}} \circ \Phi_{p_{1}, V_{1}}$ is continuous. Thus we obtain a $C^{0}$ atlas on $\mathcal{M}_{j}^{\text {int }}$. (That the topology on $\mathcal{M}_{j}^{\text {int }}$ coincides with the quotient topology is a detail we leave to the reader.)

However, the map $A: C^{j}\left(S^{1}\right) \rightarrow \operatorname{Diff}_{j}^{+}\left(S^{1}\right)$ is not differentiable; differentiating $A$ at $f$ introduces derivatives of $f$, which are only $C^{j-1}$. (This is a well-known problem with right-composition; cf. [H], Examples I.4.4.5 and I.4.4.6, pp. 91-92.) Thus our atlas is not even $C^{1}$.

(f) From [H], Corollary II.2.3.7, p. 149, the space $\mathcal{F}$ of all compact smooth submanifolds of $S^{1} \times S^{1}$ has a natural structure as a tame Fréchet manifold, and for $\Sigma \in \mathcal{F}$ there is a canonical isomorphism between $T_{\Sigma} \mathcal{F}$ and the space of $C^{\infty}$ normal vector fields along $\Sigma$ (cf. [H], Example I.4.1.7, pp. 86-87; in our case the isomorphism is canonical because we have endowed $S^{1} \times S^{1}$ with a Riemannian metric, and can use the normal exponential map along $\Sigma$ to identify a tubular neighborhood of $\Sigma$ in $S^{1} \times S^{1}$ with a neighborhood of the zero-section of the normal bundle to $\Sigma$ ). $\mathcal{M}_{\infty}^{\text {int }}$ is an open subset of $\mathcal{F}$, and as such inherits the structure of a tame Fréchet manifold. For $\Sigma \in \mathcal{M}_{\infty}^{\text {int }}$, the space of smooth normal vector fields along $\Sigma$ is naturally isomorphic to $C^{\infty}(\Sigma)$ via $\bar{f} \mathbf{N}_{\Sigma} \mapsto \bar{f}$. Equivalently, given $\Sigma$, there is a $\operatorname{Diff}^{+}\left(S^{1}\right)$-equivariant family of neighborhoods of $0,\left\{U_{p} \subset C^{\infty}\left(S^{1}\right)\right\}$, parametrized by $p \in \pi_{\overline{\mathcal{M}} \rightarrow \mathcal{M}}^{-1}(\Sigma)$, such that the pairs $\left(\bar{\Psi}_{p}\left(U_{p}\right), \bar{\Psi}_{p}^{-1}\right)$ are charts of 
$\mathcal{M}^{\text {int }}$, where $\bar{\Psi}_{p}(f)=\pi_{\widetilde{\mathcal{M}} \rightarrow \mathcal{M}}\left(p+f \mathbf{N}_{p}\right)$. Note that the maps $\bar{\Psi}_{p}$ are also $\operatorname{Diff}^{+}\left(S^{1}\right)$ equivariant: $\bar{\Psi}_{p \circ \sigma}(f \circ \sigma)=\bar{\Psi}_{p}(f)$.

The fact that $\widetilde{\mathcal{M}}_{\infty}^{\text {int }} \rightarrow \mathcal{M}_{\infty}^{\text {int }}$ is a principal Fréchet $\operatorname{Diff}^{+}\left(S^{1}\right)$-bundle over $\mathcal{M}_{\infty}^{\text {int }}$ is a special case of $[\mathrm{H}$, Example I.4.6.6, p. 99. From this principal-bundle structure, it follows that the manifold topology on $\mathcal{M}_{\infty}^{\text {int }}$ coincides with the quotient topology. Tameness of the bundle $\widetilde{\mathcal{M}}_{\infty}^{\text {int }} \rightarrow \mathcal{M}_{\infty}^{\text {int }}$ follows from the argument for $[\mathrm{H}$, Corollary II.2.3.7, p. 149. However, the proof of local triviality of this bundle -i.e. that $\widetilde{\mathcal{M}}_{\infty}^{\text {int }} \rightarrow \mathcal{M}_{\infty}^{\text {int }}$ is locally (in $\mathcal{M}_{\infty}^{\text {int }}$ ) equivariantly diffeomorphic to \{open set in $\left.\mathcal{M}_{\infty}^{\text {int }}\right\} \times \operatorname{Diff}^{+}\left(S^{1}\right)$-is not quite as simple as its omission from $\underline{\mathrm{H}}$ would suggest; the problem is showing that the "obvious" diffeomorphisms are in fact diffeomorphisms (and tamely smooth). We will not give the details here for the $\operatorname{Diff}^{+}\left(S^{1}\right)$-bundle $\widetilde{\mathcal{M}}_{\infty}^{\text {int }} \rightarrow \mathcal{M}_{\infty}^{\text {int }}$, since we do not rely on this bundle structure for any of our results; however, we give the details below for the $S^{1}$-bundle $\widetilde{\mathcal{M}}_{\infty}^{\text {int }, c} \rightarrow \mathcal{M}_{\infty}^{\text {int }}$, on whose structure we do rely.

Let $\operatorname{proj}_{1,2}: C^{\infty}\left(S^{1}\right) \oplus \mathbf{R} \oplus C_{0}^{\infty} \rightarrow C^{\infty}\left(S^{1}\right) \oplus \mathbf{R}$ denote the projection $(f, a, g) \mapsto$ $(f, a)$, and $\operatorname{proj}_{1}: C^{\infty}\left(S^{1}\right) \oplus \mathbf{R} \rightarrow C^{\infty}\left(S^{1}\right)$ the projection $(f, a) \mapsto f$. The manifold $\widetilde{\mathcal{M}}_{\infty}^{\text {int,c }}$ has charts of the form $\left(U_{p}^{c},\left(\tilde{\Psi}_{p}^{c}\right)^{-1}\right)$ where, in the notation used at the end of the proof of (d),

$$
\begin{aligned}
W_{p}^{c} & =\operatorname{proj}_{1,2}\left(W_{1}(p) \cap\left(C^{\infty}\left(S^{1}\right) \oplus \mathbf{R}\right) \times\{0\}\right), \\
\tilde{\Psi}_{p}^{c}(f, a) & =\tilde{\Psi}_{p}(f, a, 0), \\
\text { and } \quad & U_{p}^{c}
\end{aligned}
$$

For each $p \in \widetilde{\mathcal{M}}_{\infty}^{\text {int, },}$, we also define

$$
V_{p}=\operatorname{proj}_{1}\left(U_{p}^{c}\right)=\left\{f \in C^{\infty}\left(S^{1}\right) \mid\|f\|_{2}<\epsilon(p)\right\}
$$

and

$$
\begin{aligned}
\Psi_{p}^{c}: V_{p} & \rightarrow \widetilde{\mathcal{M}}_{\infty}^{\mathrm{int}, c}, \\
\Psi_{p}^{c}(f) & =\tilde{\Psi}_{p}^{c}(f, 0)=\tilde{\Psi}_{p}((f, 0), 0)=\pi_{\widetilde{\mathcal{M}} \rightarrow \widetilde{\mathcal{M}}^{c}}\left(p+H_{p}(f, 0)\right) .
\end{aligned}
$$

For the remainder of this proof, let $\pi=\pi_{\widetilde{\mathcal{M}}^{c} \rightarrow \mathcal{M}}$. Let $\Sigma \in \mathcal{M}^{\text {int }}$ and let $p$ be a constant-speed parametrization of $\Sigma$. We define a map $P=P_{p}: V_{p} \times S^{1} \rightarrow \widetilde{\mathcal{M}}_{\infty}^{\text {int }, c}$ by $P(f, a)=\tau_{a}\left(\Psi_{p}^{c}(f)\right)$. Clearly $\pi \circ(P(f, a))=\Psi_{p}^{c}(f)$ and $P$ is $S^{1}$-equivariant: $P\left(f, a+a^{\prime}\right)=\tau_{a^{\prime}}(P(f, a))$. It remains to show that (possibly after shrinking $\left.V_{p}\right) P$ is a diffeomorphism onto $\pi^{-1}\left(V_{p}\right)$.

Every curve $\Sigma^{\prime}$ lying in a sufficiently small $C^{1}$-neighborhood of $\Sigma$ admits a unique parametrization of the form $t \mapsto q(t):=\left(p+f N_{p}\right)(t)$ with $\|f\|_{1}$ small. In the derivation of (4.22), if we replace $H_{p}(f, a)$ by $f \mathbf{N}_{p}$ we still get a linear bound $\left\|\sigma_{q}-\mathrm{id}\right\| \leq C\|f\|_{1}$, and since $|\dot{q}| \leq C$ this implies that $\pi(q)$, a constantspeed parametrization of $\Sigma^{\prime}$, is a small $C^{1}$-distance from $p$. Every constant-speed parametrization of $\Sigma^{\prime}$ is of the form $\tau_{a}(\pi(q))$ for some $a \in S^{1}$. It follows that, after shrinking $\epsilon=\epsilon(p)$ in (4.13) if necessary, $P$ maps $V_{p} \times S^{1}$ onto $\pi^{-1}\left(V_{p}\right)$.

Next we show that $P$ is one-to-one. For this, it suffices to show that no $S^{1}$-orbit intersects the "slice"

$$
\mathcal{H}_{p}^{c}:=\operatorname{image}\left(\Psi_{p}^{c}\right)
$$

twice. Temporarily use elements of $\left[-\frac{1}{2},-\frac{1}{2}\right)$ to represent elements of $S^{1}$. Since $|\dot{p}| \equiv \ell_{p}$, for all $a \in S^{1}$ with $|a|<\epsilon / \ell_{p}$ we have $\tau_{a}(p)=p+X$ for some $X$ with $\|X\|_{2}<\epsilon$. If some $S^{1}$-orbit intersects $\mathcal{H}_{p}^{c}$ twice then there exist $f_{1}, f_{2} \in V_{p}$ and 
nonzero $a \in S^{1}$ such that $\tau_{a}\left(\hat{q}_{1}\right)=\hat{q}_{2}$, where $q_{i}=\Psi_{p}^{c}\left(f_{i}\right)$ and $\hat{q}_{i}=\pi_{\widetilde{\mathcal{M}} \rightarrow \widetilde{\mathcal{M}}^{c}}\left(q_{i}\right)=$ $q_{i} \circ \sigma_{q_{i}}$. If $|a|<\epsilon / \ell_{p}$ then $q_{i}-p$ and $\hat{q}_{i}-p$ are well-defined vector-valued functions, and using (4.22) we have

(4.66)

$$
\begin{aligned}
0= & \tau_{a}\left(\hat{q}_{1}\right)-\hat{q}_{2} \\
= & \left(\tau_{a}\left(\hat{q}_{1}\right)-\hat{q}_{1}\right)+\left(q_{1} \circ \sigma_{q_{1}}-q_{1} \circ \sigma_{q_{2}}\right)+\left(\left(q_{1}-q_{2}\right) \circ \sigma_{q_{2}}-\left(q_{1}-q_{2}\right)\right)+\left(q_{1}-q_{2}\right) \\
= & \left(a \mathbf{T}_{\hat{q}_{1}}+O\left(a^{2}\right)\right)+O\left(\left\|\dot{q}_{1}\right\|_{0}\left\|\sigma_{q_{1}}-\sigma_{q_{2}}\right\|_{0}\right)+O\left(\left\|\left(q_{1}-q_{2}\right)\right\|_{0}\left\|\sigma_{q_{2}}-\mathrm{id}\right\|_{0}\right) \\
& \quad+\left(q_{1}-q_{2}\right) .
\end{aligned}
$$

Let $X=q_{1}-q_{2}$. Then $X=H_{p}\left(f_{1}-f_{2}, 0\right),\|\dot{X}\|_{0} \leq C\left\|f_{1}-f_{2}\right\|_{1}$ by (4.17), and from (4.22) we have $\left\|\sigma_{q_{2}}-\mathrm{id}\right\|_{0}=O\left(\left\|f_{2}\right\|_{1}^{2}\right)=O\left(\epsilon^{2}\right)$. Using (4.29) we have

$$
\begin{aligned}
\sigma_{q_{1}}(t)-\sigma_{q_{2}}(t) & =\int_{0}^{1} \frac{d}{d s} \sigma_{q_{2}+s X}(t) d s \\
& =\int_{0}^{1}\left(\left.D G_{2}\right|_{q_{2}+s X}(X)(t)\right) d s \\
& =-\int_{0}^{1} \frac{\hat{\alpha}_{q_{2}+s X}(X)\left(\sigma_{q_{2}+s X}(t)\right)}{\left|\left(q_{2}+s X\right)^{\circ}\left(\sigma_{q_{2}+s X}(t)\right)\right|} d s .
\end{aligned}
$$

Since $\hat{\alpha}_{q_{2}+s X}(X)=\alpha_{p+H_{p}\left(s f_{1}+(1-s) f_{2}, 0\right)}\left(f_{1}-f_{2}, 0\right)$, from (4.58) we have

$$
\left\|\hat{\alpha}_{q_{2}+s X}(X)\right\|_{0} \leq C\left\|(1-s) f_{2}+s f_{1}\right\|_{2}\left\|f_{1}-f_{2}\right\|_{0} \leq C \epsilon\left\|f_{1}-f_{2}\right\|_{0} .
$$

Since $\left|\left(q_{2}+s X\right)^{\cdot}\right| \geq \ell_{p} / 2$ pointwise, it follows that $\left\|\sigma_{q_{1}}-\sigma_{q_{2}}\right\|_{0}=O\left(\epsilon\left\|f_{1}-f_{2}\right\|_{0}\right)$, and since $\left\|\dot{q}_{1}\right\|_{0} \leq C\left(1+\left\|f_{1}\right\|_{1}\right) \leq C$, equations (4.66) and (4.67) then imply that

$$
0=H_{p}\left(f_{2}-f_{1}, a\right)+a\left(\mathbf{T}_{\hat{q}_{1}}-\mathbf{T}_{p}\right)+O\left(a^{2}\right)+O\left(\epsilon\left\|f_{1}-f_{2}\right\|_{1}\right) .
$$

From (4.20) and (4.17) we find

$$
\mathbf{T}_{q_{1}}-\mathbf{T}_{p}=\frac{\dot{q}_{1}}{\ell_{q_{1}}}-\frac{\dot{p}}{\ell_{p}}=O\left(\|f\|_{1}^{2}\right) \mathbf{T}_{p}+\frac{\left(\dot{f}+\dot{\theta}_{p} \hat{\lambda}_{p}(f)\right)}{\ell_{q_{1}}} \mathbf{N}_{p}
$$

and hence

$$
H_{p}\left(f_{1}-f_{2}, a\right)=a\left(O\left(\epsilon^{2}\right) \mathbf{T}_{p}+O(\epsilon) \mathbf{N}_{p}\right)+O\left(a^{2}\right)+O\left(\epsilon\left\|f_{1}-f_{2}\right\|_{1}\right) .
$$

Taking the $\mathbf{T}_{p}$-component of this equation and integrating over $S^{1}$, using (4.9) and recalling that $|a|<\epsilon / \ell_{p}$, we find $a=a O\left(\epsilon^{2}\right)$, a contradiction once we take $\epsilon$ sufficiently small.

By the foregoing, after shrinking $\epsilon$ there exists $\delta>0$ such that if some $S^{1}$-orbit intersects $\mathcal{H}_{p}^{c}$ twice, then there exist $a \in S^{1}$ and $f_{i}, q_{i}, \hat{q}_{i}$ as above with $\operatorname{dist}(a, 0) \geq \delta$, $\tau_{a}\left(\hat{q}_{1}\right)=\hat{q}_{2}$, and $\left\|\sigma_{q_{i}}-\mathrm{id}\right\|_{0}<\delta / 2$. Since $p: S^{1} \rightarrow S^{1} \times S^{1}$ is an embedding, there exists $\epsilon_{1}>0$ such that if $\operatorname{dist}\left(p\left(t_{1}\right), p(0)\right)<\epsilon_{1}$, then $\operatorname{dist}\left(t_{1}, 0\right)<\delta / 2$. By (4.17) we can shrink $\epsilon$, if necessary, so that for all $f \in V_{p}$ we have $\left\|H_{p}(f, 0)\right\|_{0}<$ $\epsilon_{1} / 2$. Evaluating " $\tau_{a}\left(\hat{q}_{1}\right)=\hat{q}_{2}$ " at $t=0$, we have $p\left(\sigma_{q_{1}}(a)\right)+H_{p}\left(f_{1}, 0\right)\left(\sigma_{q_{1}}(a)\right)=$ $p(0)+H_{p}\left(f_{2}, 0\right)(0)$, implying $\operatorname{dist}\left(p\left(\sigma_{q_{1}}(a)\right), p(0)\right)<\epsilon_{1}$, and hence $\operatorname{dist}\left(\sigma_{q_{1}}(a), 0\right)<$ $\delta / 2$. But dist $\left(\sigma_{q_{1}}(a), 0\right) \geq \operatorname{dist}(a, 0)-\operatorname{dist}\left(a, \sigma_{q_{1}}(a)\right)>\delta-\left\|\sigma_{q_{1}}-\mathrm{id}\right\|_{0}>\delta / 2$, a contradiction. Hence $P$ is one-to-one. 
Therefore $P: V_{p} \times S^{1} \rightarrow \pi^{-1}\left(V_{p}\right)$ is bijective, hence invertible; it remains to show that $P$ and $P^{-1}$ are tamely smooth. From the tamely-smoothness shown in (d) of our atlas for $\widetilde{\mathcal{M}}_{\infty}^{\text {int,c }}$, it is easy to check that $P$ is tamely smooth. We next check invertibility of the derivative of $\left(\tilde{\Psi}_{P(f, a)}^{c}\right)^{-1} \circ P$, the chart-representative of $P$.

Let $(f, a) \in V_{p} \times S^{1}$, let $q=\tilde{\Phi}_{p}(f, 0)$, and let $\hat{q}=q \circ \sigma_{q}$. From (4.32) we have $\dot{q}=b_{8}(f) \mathbf{N}_{p}+c_{f} \mathbf{T}_{p}$, where $b_{8}(f)=\dot{f}+\hat{\lambda}_{p}(f) \dot{\theta}_{p}$ and $c_{f}=\ell_{p}-\left\langle f \dot{\theta}_{p}\right\rangle$. For some $f_{2} \in C^{\infty}\left(S^{1}\right), a_{2} \in \mathbf{R}$ we have

$$
\left.D\left(\left(\tilde{\Psi}_{P(f, a)}^{c}\right)^{-1} \circ P\right)\right|_{(f, a)}\left(\begin{array}{c}
f_{1} \\
a_{1}
\end{array}\right)=\left(\begin{array}{c}
\tau_{a}\left(f_{2} \circ \sigma_{q}\right) \\
a_{2}
\end{array}\right)
$$

i.e. $\left.D P\right|_{(f, a)}\left(\begin{array}{c}f_{1} \\ a_{1}\end{array}\right)=H_{P(f, a)}\left(\tau_{a}\left(f_{2} \circ \sigma_{q}\right), a_{2}\right)$, where $q=P(f, 0)$. Since $P(f, a)=$ $p+\tau_{a}(G(f, 0,0))$ (see (4.23) $)$ we can use the calculations in (d) to determine $f_{2}, a_{2}$. Noting that $p+G(f, 0,0)=\hat{q}$ and using (4.30)-4.31), we have

$$
\begin{aligned}
\left.(D P)\right|_{(f, a)}\left(\begin{array}{c}
f_{1} \\
a_{1}
\end{array}\right) & =\tau_{a}\left(\left.(D G)\right|_{(f, 0,0)}\left(\begin{array}{c}
f_{1} \\
0 \\
0
\end{array}\right)\right)+a_{1} \tau_{a}(\hat{q}) \\
& =\tau_{a}\left(\left[H_{p}\left(f_{1}, 0\right)-\alpha_{(p, f, 0)}\left(f_{1}, 0\right) \mathbf{T}_{q}\right] \circ \sigma_{q}\right)+a_{1} \tau_{a}\left(\ell_{q} \mathbf{T}_{\hat{q}}\right) .
\end{aligned}
$$

Since $\mathbf{T}_{q}=|\dot{q}|^{-1}\left(b_{8}(f) \mathbf{N}_{p}+c_{f} \mathbf{T}_{p}\right)$, we have $\mathbf{N}_{q}=|\dot{q}|^{-1}\left(-c_{f} \mathbf{N}_{p}+b_{8}(f) \mathbf{T}_{p}\right)$, and therefore $\mathbf{T}_{p}=|\dot{q}|^{-1}\left(-b_{8}(f) \mathbf{N}_{q}+c_{f} \mathbf{T}_{q}\right), \mathbf{N}_{p}=|\dot{q}|^{-1}\left(c_{f} \mathbf{N}_{q}+b_{8}(f) \mathbf{T}_{q}\right)$. Substituting these relations into $H_{p}\left(f_{1}, 0\right)$, and noting that $\tau_{a}\left(\mathbf{N}_{q} \circ \sigma_{q}\right)=\tau_{a}\left(\mathbf{N}_{\hat{q}}\right)=\mathbf{N}_{P(f, a)}$ and $\tau_{a}\left(\mathbf{T}_{q} \circ \sigma_{q}\right)=\tau_{a}\left(\mathbf{T}_{\hat{q}}\right)=\mathbf{T}_{P(f, a)}$, the right-hand side of (4.69) becomes

$$
\begin{aligned}
& \tau_{a}\left(\left[\frac{c_{f} f_{1}-b_{8}(f) \hat{\lambda}_{p}\left(f_{1}\right)}{|\dot{q}|}\right] \circ \sigma_{q}\right) \mathbf{N}_{P(f, a)} \\
& +\left(\ell_{q} a_{1}+\tau_{a}\left(\left[-\alpha_{q}\left(f_{1}, 0\right)+\frac{b_{8}(f) f_{1}}{|\dot{q}|}\right] \circ \sigma_{q}\right)\right) \mathbf{T}_{P(f, a)} .
\end{aligned}
$$

Because $\left.(D P)\right|_{(f, a)}\left(\begin{array}{l}f_{1} \\ a_{1}\end{array}\right)$ is tangent to $\widetilde{\mathcal{M}}^{\text {int,c }}$, (4.70) is automatically of the form $f_{3} \mathbf{N}_{P(f, a)}+\left(\hat{\lambda}_{P(f, a)}\left(f_{3}\right)+a_{2}\right) \mathbf{T}_{P(f, a)}$, where $a_{2}$ must be the average value of the coefficient of $\mathbf{T}_{P(f, a)}$ in (4.70). Thus we conclude that

$$
\begin{aligned}
f_{2} & =\frac{c_{f} f_{1}-b_{8}(f) \hat{\lambda}_{p}\left(f_{1}\right)}{|\dot{q}|} \\
\text { and } \quad a_{2} & =\ell_{q} a_{1}+\left\langle\left[-\alpha_{q}\left(f_{1}, 0\right)+\frac{b_{8}(f) f_{1}}{|\dot{q}|}\right] \circ \sigma_{q}\right\rangle \text {. }
\end{aligned}
$$

We show $\left.D\left(\left(\tilde{\Psi}_{P(f, a)}^{c}\right)^{-1} \circ P\right)\right|_{(f, a)}$ is invertible for $(f, a)$ near $(0,0)$ by a strategy similar to the one used in (d). This derivative is invertible iff the map $A_{(f, a)}$ : $\left(\begin{array}{c}f_{1} \\ a_{1}\end{array}\right) \mapsto\left(\begin{array}{c}f_{2} \\ a_{2}\end{array}\right)$ is invertible, where $f_{2}, a_{2}$ are given by (4.71)-(4.72). Define $S_{(f, a)}$ by $A_{(f, a)}=A_{(0,0)}+S_{(f, a)}$. These linear maps all extend by the formulas above to maps $C^{0}\left(S^{1}\right) \oplus \mathbf{R} \rightarrow C^{0}\left(S^{1}\right) \oplus \mathbf{R}$; we denote the extensions by the same letters. The map $A_{(0,0)}$ is simply $\left(\begin{array}{c}f_{1} \\ a_{1}\end{array}\right) \mapsto\left(\begin{array}{c}f_{1} \\ \ell_{p} a_{1}\end{array}\right)$. Using the estimates in (d), 
one easily finds that

$$
\left\|S_{(f, a)} A_{0,0}^{-1}\left(\begin{array}{c}
f_{1} \\
a_{1}
\end{array}\right)\right\|_{0} \leq C\|f\|_{2}\left\|\left(\begin{array}{c}
f_{1} \\
a_{1}
\end{array}\right)\right\|_{0} .
$$

Hence if $\|f\|_{2}$ is sufficiently small, the operator norm of $S_{(f, a)} A_{(0,0)}^{-1}$ as an operator on $C^{0}\left(S^{1}\right) \oplus \mathbf{R}$ is less than 1 , and $A_{(f, a)}$ inverts from $C^{0}\left(S^{1}\right) \oplus \mathbf{R}$ to $C^{0}\left(S^{1}\right) \oplus \mathbf{R}$. If $f_{3} \in C^{\infty}, a_{3} \in \mathbf{R}$, and $\left(\begin{array}{c}f_{1} \\ a_{1}\end{array}\right)=A_{(f, a)}^{-1}\left(\begin{array}{c}f_{3} \\ a_{3}\end{array}\right)$, then $f_{1}=c_{f}^{-1}\left(|\dot{q}| f_{3}+b_{8}(f) \hat{\lambda}_{p}\left(f_{1}\right)\right)$. Since $f, q$, and $b_{8}(f)$ are $C^{\infty}$, it follows that if $f_{1} \in C^{j}$, then the right-hand side of this equation is in $C^{j+1}$, and hence $f_{1} \in C^{\infty}$ by induction. Thus $A_{(f, a)}^{-1}$ preserves $C^{\infty}\left(S^{1}\right) \oplus \mathbf{R}$, and it follows that $\left.D\left(\left(\tilde{\Psi}_{P(f, a)}^{c}\right)^{-1} \circ P\right)\right|_{(f, a)}$ is invertible; hence so is $\left.D\left(\tilde{\Psi}^{p^{\prime}}\right)^{-1} \circ P\right)_{(f, a)}$ for any fixed $p^{\prime}$ sufficiently near $P(f, a)$. We also find that for all $n \geq 1$ and all $\left(f^{\prime}, a^{\prime}\right)$ sufficiently close to $(f, a)$ in the $(n+1)$-norm,

$$
\left\|S_{\left(f^{\prime}, a^{\prime}\right)} A_{(0,0)}^{-1}-S_{(f, a)} A_{(0,0)}^{-1}\right\|_{\mathrm{op}, n} \leq C(f, a, n)\left\|\left(\begin{array}{c}
f^{\prime} \\
a^{\prime}
\end{array}\right)-\left(\begin{array}{c}
f \\
a
\end{array}\right)\right\|_{n+1} .
$$

Hence, by an argument similar to that used in (d), the map $\left((f, a),\left(\begin{array}{c}f_{1} \\ a_{1}\end{array}\right)\right) \mapsto$ $\left.D\left(\left(\tilde{\Psi}^{p^{\prime}}\right)^{-1} \circ P\right)\right|_{(f, a)}\left(\begin{array}{c}f_{1} \\ a_{1}\end{array}\right)$ is continuous and tame, hence tamely smooth (since $P$ is). Hence $P$ is a tamely smooth diffeomorphism.

Remark 4.2. At each $\Sigma \in \mathcal{M}^{\text {int }}$, the bundle structures $\widetilde{\mathcal{M}}_{\infty}^{\text {int }} \rightarrow \mathcal{M}^{\text {int }}$ and $\widetilde{\mathcal{M}}_{\infty}^{\text {int,c }} \rightarrow$ $\mathcal{M}^{\text {int }}$ give us different natural identifications of the tangent space $T_{\Sigma} \mathcal{M}^{\text {int }}$ with subspaces of $T_{p} \mathcal{M}^{\text {int }}$ for $p \in \pi_{\widetilde{\mathcal{M}}^{c} \rightarrow \mathcal{M}}^{-1}(\Sigma)$. The $\operatorname{Diff}^{+}\left(S^{1}\right)$-bundle leads to the identification of $T_{\Sigma} \mathcal{M}^{\text {int }} \longleftrightarrow \tilde{T}_{p}^{(0)} \mathcal{M}_{\infty}^{\text {int }}=\left\{f \mathbf{N}_{p} \mid f \in C^{\infty}\left(S^{1}\right)\right\} \subset T_{p} \widetilde{\mathcal{M}}^{\text {int }}$, and we can define a corresponding slice $\mathcal{H}_{p}=\left\{p+f \mathbf{N}_{p} \mid f \in C^{\infty}\left(S^{1}\right)\right\}$ whose tangent space at $p$ is this subspace. The family of subspaces, the family of slices, and the family of isomorphisms $T_{p} \mathcal{H}_{p} \rightarrow T_{\Sigma} \mathcal{M}^{\text {int }}$ are all $\operatorname{Diff}^{+}\left(S^{1}\right)$-invariant along $\pi_{\widetilde{\mathcal{M}} \rightarrow \mathcal{M}}^{-1}(\Sigma)$, and hence $S^{1}$-invariant along $\pi_{\mathcal{\mathcal { M }}^{c} \rightarrow \mathcal{M}}^{-1}(\Sigma)$. Similarly, the $S^{1}$-bundle gives us an $S^{1}$-invariant family of isomorphisms from the $S^{1}$-invariant family of subspaces image $\left(H_{p}(\cdot, 0)\right)$ | $\left.p \in \pi_{\widetilde{\mathcal{M}}^{c} \rightarrow \mathcal{M}}^{-1}(\Sigma)\right\}$ to $T_{\Sigma} \mathcal{M}^{\text {int }}$; from (4.10) we have $H_{\tau_{a}(p)}\left(\tau_{a}(f), 0\right)=\tau_{a}\left(H_{p}(f, 0)\right)$. It then follows from Proposition 2.5 that $\Psi_{\tau_{a}(p)}^{c}\left(\tau_{a}(f)\right)=\pi_{\widetilde{\mathcal{M}} \rightarrow \widetilde{\mathcal{M}}^{c}}\left(\tau_{a}\left(p+H_{p}(f, 0)\right)\right)$ $=\tau_{a}\left(\Psi_{p}^{c}(f)\right)$, and therefore that the family of slices $\left\{\mathcal{H}_{p}^{c}\right\}$ is $S^{1}$-invariant as well. Letting $\Psi_{p}=\pi_{\widetilde{\mathcal{M}}^{c} \rightarrow \mathcal{M}} \circ \Psi_{p}^{c}: V_{p} \rightarrow \mathcal{M}^{\text {int }}$, the collection $\left\{\Psi_{p}\left(V_{p}\right), \Psi_{p}^{-1}\right\}_{p \in \widetilde{\mathcal{M}}^{\text {int }, c}}$ is therefore a natural atlas for $\mathcal{M}^{\text {int }}$ (different from, but smoothly tamely equivalent to, the atlas $\left\{\bar{\Psi}_{p}\left(U_{p}\right), \bar{\Psi}_{p}^{-1}\right\}_{p \in \widetilde{\mathcal{M}}^{\text {int }, c}}$ described at the start of the proof of part (f) above). The maps $\Psi_{p}^{c} \circ \Psi_{p}^{-1}$ are the local sections $s$ alluded to in the proof of G1, Lemma 3.1].

Remark 4.3. For use in G1] we make the expression for $a_{2}$ more explicit. First, since $\left(\sigma_{q}^{-1}\right)^{\cdot}=\ell_{q}^{-1}|\dot{q}|$, by a change of variables we have $\left\langle\left[b_{8}(f) f_{1} /|\dot{q}|\right] \circ \sigma_{q}\right\rangle=\left\langle b_{8}(f) f_{1}\right\rangle$. Next let $Z \in C^{\infty}\left(S^{1}, \mathbf{R}^{2}\right)$, and recalling (4.27)-(4.28) let $g(s)$ be the integrand in (4.28) and let $h(t)=\bar{\alpha}_{q}(Z)(t)=\int_{0}^{t} g(s) d s-\bar{\sigma}_{q}^{-1}(t)\langle g\rangle$. Since $\left(\sigma_{q}^{-1}\right)^{\cdot}=\left(\bar{\sigma}_{q}^{-1}\right)^{\cdot}$ and 
$h(0)=h(1)=0$, we have

$$
\begin{aligned}
\int_{0}^{1}\left(\bar{\alpha}_{q}(Z)\right)\left(\sigma_{q}(s)\right) d s & =\int_{0}^{1} h(t)\left(\bar{\sigma}_{q}^{-1}\right)^{\dot{ }}(t) d t \\
& =-\int_{0}^{1} \dot{h}(t) \bar{\sigma}_{q}^{-1}(t) d t \\
& =-\int_{0}^{1}\left(g(t)-\left(\bar{\sigma}_{q}^{-1}\right)^{\cdot}(t)\langle g\rangle\right) \bar{\sigma}_{q}^{-1}(t) d t \\
& =-\int_{0}^{1} g(t) \bar{\sigma}_{q}^{-1}(t) d t+\frac{1}{2}\langle g\rangle .
\end{aligned}
$$

We also have

$$
\left\langle\bar{\alpha}_{q}(Z)\right\rangle=\int_{0}^{1} \int_{0}^{t} g(s) d s d t-\left\langle\bar{\sigma}_{q}^{-1}\right\rangle\langle g\rangle=\int_{0}^{1}(1-s) g(s) d s-\left\langle\bar{\sigma}_{q}^{-1}\right\rangle\langle g\rangle .
$$

Since $\left\langle\bar{\sigma}_{q}^{-1}-\right.$ id. $\rangle=\left\langle\bar{\sigma}_{q}^{-1}\right\rangle-1 / 2$, combining (4.73) and (4.74) we have

$$
\begin{aligned}
\left\langle\hat{\alpha}_{q}(Z) \circ \sigma_{q}\right\rangle=\left\langle\bar{\alpha}_{q}(Z) \circ \sigma_{q}\right\rangle-\left\langle\bar{\alpha}_{q}(Z)\right\rangle & =\langle g\rangle\left\langle\bar{\sigma}_{q}^{-1}-\mathrm{id} .\right\rangle-\int_{0}^{1} g(t)\left(\bar{\sigma}_{q}^{-1}(t)-t\right) d t \\
& \left.=-\int_{0}^{1} g(t)\left(\sigma_{q}^{-1}(t)-t\right)\right) d t .
\end{aligned}
$$

From (4.41), in our current context

$$
g(t)=|\dot{q}(t)|^{-1}\left\{b_{8}(f)\left(\dot{f}_{1}+\dot{\theta}_{p} \hat{\lambda}_{p}\left(f_{1}\right)\right)+c_{f}\left\langle f_{1} \dot{\theta}_{p}\right\rangle\right\} .
$$

Hence

$$
\begin{aligned}
a_{2}= & \ell_{q} a_{1}+\ell_{q}^{-1}\left\langle b_{8}(f) f_{1}\right\rangle \\
& \left.+\int_{0}^{1}\left\{\left(\bar{\sigma}_{q}^{-1}(t)-t\right)\right) \frac{b_{8}(f)\left(\dot{f}_{1}+\dot{\theta}_{p} \hat{\lambda}_{p}\left(f_{1}\right)\right)+c_{f}\left\langle f_{1} \dot{\theta}_{p}\right\rangle}{\sqrt{b_{8}(f)^{2}+c_{f}^{2}}}(t)\right\} d t .
\end{aligned}
$$

In the proof of Theorem 2.8 we used Lemma 4.4 (see below). To prove this lemma we rely on the following interpolation inequality: for all $i, j \geq 0$ there exists a constant $C$ such that for all $f, g \in C^{\infty}\left(S^{1}\right)$,

$$
\|f\|_{i}\|g\|_{j} \leq C\left(\|f\|_{i+j}\|g\|_{0}+\|f\|_{0}\|g\|_{i+j}\right)
$$

This is a special case of $[\mathrm{H}$, Corollary II.2.2.2, p. 144, and extends by induction to a product with any number of factors:

$$
\left\|f_{1}\right\|_{n_{1}}\left\|f_{2}\right\|_{n_{2}} \ldots\left\|f_{r}\right\|_{n_{r}} \leq C\left(\left\|f_{1}\right\|_{N}\left\|f_{2}\right\|_{0} \ldots\left\|f_{r}\right\|_{0}+\left\|f_{1}\right\|_{0}\left\|f_{2}\right\|_{N} \ldots\left\|f_{r}\right\|_{0}\right.
$$

where $N=\sum_{k} n_{k}$.

The lemma below holds true on any compact Riemannian manifold, but to keep the notation simple we prove them only for $S^{1}$.

Lemma 4.4. Let $\alpha<1$. For all $n \geq 1$ there exists a constant $C$ such that for all $c_{1}>0$ and all $h \in C^{\infty}\left(S^{1}\right)$ satisfying $h(t) \geq c_{1}$ for all $t \in S^{1}$, we have

$$
\left\|h^{\alpha}\right\|_{n} \leq\left\|h^{\alpha}\right\|_{0}+C c_{1}^{\alpha-n}\|h\|_{0}^{n-1}\left\|h-c_{1}\right\|_{n} .
$$


Proof. By rescaling $h$, it suffices to establish (4.78) for $c_{1}=1$, which we henceforth assume.

Let $h_{1}=h-1$. By induction, the $n^{\text {th }}$ derivative of $h^{\alpha}$ is a linear combination, with universal coefficients, of terms of the form $h^{-\beta} \Pi_{k=1}^{r}\left(h_{1}^{\left(i_{k}\right)}\right)^{j_{k}}$, where $\beta>0$ and the integers, $r, i_{k}$, and $j_{k}$ are all $\geq 1$, and where $\sum_{k} i_{k} j_{k}=n$. Since $h \geq 1$, it suffices to show that $\left\|\Pi_{k=1}^{r}\left(h_{1}^{\left(i_{k}\right)}\right)^{j_{k}}\right\|_{0} \leq C$. Using (4.77), for all $i \geq 0, j \geq 1$, and $r \geq 1$, for all $f \in C^{\infty}\left(S^{1}\right)$ we have $\|f\|_{i}^{j} \leq C\|f\|_{0}^{j-1}\|f\|_{i j}$ and $\|f\|_{n_{1}} \ldots\|f\|_{n_{r}} \leq$ $C\|f\|_{0}^{r-1}\|f\|_{n_{1}+\cdots+n_{r}}$. Thus

$$
\begin{aligned}
\left\|\Pi_{k=1}^{r}\left(h_{1}^{\left(i_{k}\right)}\right)^{j_{k}}\right\|_{0} \leq \Pi_{k=1}^{r}\left\|h_{1}\right\|_{i_{k}}^{j_{k}} & \leq C \Pi_{k=1}^{r}\left\|h_{1}\right\|_{0}^{j_{k}-1}\left\|h_{1}\right\|_{i_{k} j_{k}} \\
& \leq C\left\|h_{1}\right\|_{0}^{\left(\sum j_{k}\right)-r}\left\|h_{1}\right\|_{0}^{r-1}\left\|h_{1}\right\|_{\sum i_{k} j_{k}} \\
& =C\left\|h_{1}\right\|_{0}^{\left(\sum j_{k}\right)-1}\left\|h_{1}\right\|_{n} \\
& \leq C\|h\|_{0}^{\left(\sum j_{k}\right)-1}\left\|h_{1}\right\|_{n} .
\end{aligned}
$$

The restrictions on $i_{k}$ and $j_{k}$ above imply $\sum_{k} j_{k} \leq n$, and since $\|h\|_{0} \geq 1$, it follows that $\left\|\left(h^{\alpha}\right)^{(n)}\right\|_{0} \leq C\|h\|_{0}^{n-1}\|h\|_{n}$. Since $\|h\|_{0} \geq 1$, the corresponding bounds on $\left\|\left(h^{\alpha}\right)^{(m)}\right\|_{0}$ for $1 \leq m<n$ are dominated by the bound on $\left\|\left(h^{\alpha}\right)^{(n)}\right\|_{0}$. Adding in the term $\left\|h^{a}\right\|_{0}$, (4.78) follows.

4.2. Manifolds of shapes. To set up the proof of Theorem 2.9. we first recall that every closed, immersed curve $C$ in the plane has an integer-valued rotation number $n(C)$, the number of times the unit normal winds around the unit circle (equivalently, the degree of the Gauss map), and which is given by a curvature integral: $n(C)=(2 \pi)^{-1} \int_{C} \kappa(s) d s$. From elementary complex analysis (or the general theory of plane curves), every simple, closed, oriented regular plane curve has rotation number \pm 1 , the sign depending on whether the orientation is clockwise or counterclockwise.

If $\kappa \in C^{0}\left(S^{1}\right)$ and $(2 \pi)^{-1} \int_{0}^{1} \kappa(t) d t \in \mathbf{Z}$ then the map $\mathbf{R} \rightarrow S^{1}$,

$$
t \mapsto(2 \pi)^{-1} \omega_{\kappa}(t) \bmod 1
$$

(see (4.1)), determines a well-defined map $S^{1} \rightarrow S^{1}$ that we will also denote by $(2 \pi)^{-1} \omega_{\kappa}$. If $\kappa$ is the curvature function of a unit-speed parametrized plane curve $\gamma$ (whether or not simple or closed), then as a real-valued function $\omega_{\kappa}(t)$ is the change in angle of the unit tangent vector as one moves along $\gamma$ from $\gamma(0)$ to $\gamma(t)$.

The following lemma completely characterizes normalized curvature functions.

Lemma 4.5. A function $\kappa \in C^{j}\left(S^{1}\right)(0 \leq j \leq \infty)$ is a normalized $C^{j}$ curvature function if and only if it satisfies the following four constraints:

(1) $\int_{0}^{1} \kappa(s) d s=2 \pi$;

(2) $\int_{0}^{1} \cos \omega_{\kappa}(t) d t=0$;

(3) $\int_{0}^{1} \sin \omega_{\kappa}(t) d t=0$; and

(4) the map $S^{1} \rightarrow \mathbf{R}^{2}, t \mapsto\left(\int_{0}^{t} \cos \omega_{\kappa}(s) d s, \int_{0}^{t} \sin \omega_{\kappa}(s) d s\right)$, is injective.

Proof. First suppose $\kappa$ is a normalized $C^{j}$ curvature function, and let $C$ be a normalized $C^{j+2}$ plane curve whose canonical parametrization $\gamma:=\gamma_{C}$ has curvature function $\kappa$. Since $C$ is simple, closed, and oriented counterclockwise, its rotation number is 1 ; therefore $\kappa$ satisfies constraint (1) above. Since $C$ is normalized, $\gamma(0)=(0,0)$ and the velocity vector $\dot{\gamma}(t)$ is precisely the unit tangent 
vector $\left(\cos \omega_{\kappa}(t), \sin \omega_{\kappa}(t)\right)$. Thus if we write $\gamma(t)=(x(t), y(t))$, then $x(t)=$ $\int_{0}^{t} \cos \omega_{\kappa}(s) d s$ and $y(t)=\int_{0}^{t} \sin \omega_{\kappa}(s) d s$. Since $C$ is closed, $\kappa$ must satisfy constraints (2) and (3) above, and since $C$ is simple, constraint (4) as well.

Conversely, suppose that $\kappa$ satisfies constraints (1)-(4). Define $x(t)=$ $\int_{0}^{t} \cos \omega_{\kappa}(s) d s, y(t)=\int_{0}^{t} \sin \omega_{\kappa}(s) d s$, and $\gamma_{\kappa}(t)=(x(t), y(t))$. Then $\gamma_{\kappa}$ parametrizes a $C^{j+2}$ oriented curve $C$, closed because of constraints (2) and (3), and simple because of constraint (4). A simple closed curve $C$ must have rotation number \pm 1 , depending on its orientation; constraint (1) implies that the orientation is counterclockwise. Furthermore we have $\gamma_{\kappa}(0)=(0,0), \gamma_{\kappa}^{\prime}(0)=(1,0)$, and $\left\|\gamma_{\kappa}^{\prime}(t)\right\| \equiv 1$, so $C$ is normalized and $\gamma_{\kappa}$ is its canonical parametrization. Thus $\kappa$ is a normalized $C^{j}$ curvature function.

Proof of Theorem 2.9. For $0 \leq j \leq \infty$ let $\widetilde{\mathcal{K}}_{j}^{1}$ denote the subspace of functions $\kappa \in C^{j}\left(S^{1}\right)$ satisfying constraint (1) of Lemma 4.5, $\widetilde{\mathcal{K}}_{j}^{1}$ is an affine subspace of $C^{j}\left(S^{1}\right)$, so we can canonically identify its tangent space at any point with a subspace of $C^{j}\left(S^{1}\right)$, specifically the closed subspace

$$
T_{\kappa} \widetilde{\mathcal{K}}_{j}^{1}=C_{0}^{j}\left(S^{1}\right):=\left\{f \in C^{j}\left(S^{1}\right) \mid\langle f\rangle=0\right\}
$$

(see (2.20) ). The condition " $s \mapsto \gamma(s)$ is injective" is an open condition in the $C^{2}$-topology on the space of parametrized plane curves $\gamma$, and the map $\widetilde{\mathcal{K}}_{j}^{1} \rightarrow$ $C^{j+2}\left(S^{1}, \mathbf{R}^{2}\right), \kappa \mapsto \gamma_{\kappa}$, is continuous, so constraint (4) in Lemma 4.5 is an open condition on $\widetilde{\mathcal{K}}_{j}^{1}$. Thus the subset $\widetilde{\mathcal{K}}_{j}^{1, \text { simp }}$ of functions $\kappa \in \widetilde{\mathcal{K}}_{j}^{1}$ satisfying this constraint is an open subset of $\widetilde{\mathcal{K}}_{j}^{1}$.

(a) (the case $j<\infty)$ As a relatively open subset of a closed affine subspace of a Banach space, $\widetilde{\mathcal{K}}_{j}^{1, \text { simp }} \subset \widetilde{\mathcal{K}}_{j}^{1}$ is a smooth Banach manifold. Define $F: \widetilde{\mathcal{K}}_{j}^{1, \text { simp }} \rightarrow \mathbf{R}^{2}$ by

$$
F(\kappa)=\left(\begin{array}{c}
\int_{0}^{1} \cos \omega_{\kappa}(s) d s \\
\int_{0}^{1} \sin \omega_{\kappa}(s) d s
\end{array}\right)=\left(\begin{array}{c}
\left\langle\cos \circ \omega_{k}\right\rangle \\
\left\langle\sin \circ \omega_{\kappa}\right\rangle
\end{array}\right) .
$$

$F$ is smooth and (by Lemma 4.5) $F^{-1}(0,0)=\widetilde{\mathcal{K}}_{j}$, so to prove that $\widetilde{\mathcal{K}}_{j}$ is a smooth manifold it suffices to prove that $(0,0)$ is a regular value of $F$ (in the Banachmanifold sense).

Let $\langle\cdot, \cdot\rangle$ denote the $L^{2}$ inner product on $C^{j}\left(S^{1}\right) ;\langle f, g\rangle:=\langle f g\rangle$. The derivative of $F$ is given by

$$
\left.D F\right|_{\kappa}(f)=\left(\begin{array}{c}
-\left\langle\left(\sin \circ \omega_{k}\right), \omega_{f}\right\rangle \\
\left\langle\left(\cos \circ \omega_{k}\right), \omega_{f}\right\rangle
\end{array}\right)
$$

Now fix $\kappa \in F^{-1}(0,0) . \widetilde{\mathcal{K}}_{j+1}^{1, \text { simp }}$ is dense in $\widetilde{\mathcal{K}}_{j}^{1, \text { simp }}$, so, with $\epsilon$ to be determined later, let $\kappa_{1} \in \widetilde{\mathcal{K}}_{j+1}^{1 \text {,simp }}$ be such that $\left\|\kappa-\kappa_{1}\right\|_{0}<\epsilon$; we then also have $\| \omega_{\kappa}-$ $\omega_{\kappa_{1}}\left\|_{0}<\epsilon,\right\| \sin \circ \omega_{\kappa}-\sin \circ \omega_{\kappa_{1}} \|_{0}<\epsilon$, and $\left\|\cos \circ \omega_{\kappa}-\cos \circ \omega_{\kappa_{1}}\right\|_{0}<\epsilon$. Let $g_{1}=$ $-\kappa_{1} \sin \circ \omega_{\kappa_{1}}, g_{2}=\kappa_{1} \cos \circ \omega_{\kappa_{1}}$, and for $a, b \in \mathbf{R}$, let $f_{a, b}=\left(a g_{1}+b g_{2}\right)^{\prime}$ (where "prime" denotes derivative with respect to the normalized arclength parameter). Since $\kappa_{1} \in \widetilde{\mathcal{K}}_{j+1}$, we have $f_{a, b} \in C_{0}^{j}\left(S^{1}\right)$, and $\omega_{f_{a, b}}=a g_{1}+b g_{2}-b \kappa_{1}(0)$. Since 
$\kappa \in F^{-1}(0,0)$, the integral of any constant against $\sin \circ \omega_{\kappa}$ or $\cos \circ \omega_{\kappa}$ is 0 ; hence

$$
\begin{aligned}
\left.D F\right|_{\kappa}\left(f_{a, b}\right)= & \left(\begin{array}{c}
-\left\langle\left(\sin \circ \omega_{\kappa}\right), a g_{1}+b g_{2}\right\rangle \\
\left\langle\left(\cos \circ \omega_{\kappa}\right), a g_{1}+b g_{2}\right\rangle
\end{array}\right) \\
= & \left(\begin{array}{c}
-\left\langle\left(\sin \circ \omega_{k_{1}}\right), a g_{1}+b g_{2}\right\rangle \\
\left\langle\left(\cos \circ \omega_{\kappa_{1}}\right), a g_{1}+b g_{2}\right\rangle
\end{array}\right) \\
& \quad+\left(\begin{array}{c}
-\left\langle\sin \circ \omega_{k}-\sin \circ \omega_{\kappa_{1}}, a g_{1}+b g_{2}\right\rangle \\
\left\langle\left(\cos \circ \omega_{\kappa}-\cos \circ \omega_{\kappa_{1}}\right), a g_{1}+b g_{2}\right\rangle
\end{array}\right) .
\end{aligned}
$$

Since $\kappa_{1}=\omega_{\kappa_{1}}^{\prime}$, we have

$$
\begin{aligned}
-\left\langle\left(\sin \circ \omega_{\kappa_{1}}\right), a g_{1}+b g_{2}\right\rangle & =\int_{0}^{1} \sin \left(\omega_{\kappa_{1}}(s)\right)\left[a \sin \left(\omega_{\kappa_{1}}(s)\right)-b \cos \left(\omega_{\kappa_{1}}(s)\right)\right] \omega_{\kappa_{1}}^{\prime}(s) d s \\
& =\int_{0}^{2 \pi}\left(a \sin ^{2} u-b \sin u \cos u\right) d u=\pi a
\end{aligned}
$$

similarly the second component of the first vector on the right-hand side of (4.81) is $\pi b$. The second vector on the right-hand side of (4.81) has norm at most

$$
\begin{array}{r}
\left(\left\|\sin \circ \omega_{\kappa}-\sin \circ \omega_{\kappa_{1}}\right\|_{0}+\left\|\cos \circ \omega_{\kappa}-\cos \circ \omega_{\kappa_{1}}\right\|_{0}\right)\left\|a g_{1}+b g_{2}\right\|_{0}<2 \epsilon(|a|+|b|)\left\|\kappa_{1}\right\|_{0} \\
<2 \epsilon(|a|+|b|)\left(\|\kappa\|_{0}+\epsilon\right) .
\end{array}
$$

From this it follows that if we take $\epsilon$ sufficiently small, and define $V_{\kappa, \kappa_{1}}=$ $\operatorname{span}\left\{g_{1}^{\prime}, g_{2}^{\prime}\right\} \subset C_{0}^{j}\left(S^{1}\right)$, then the restriction of $\left.D F\right|_{\kappa}$ to $V_{\kappa, \kappa_{1}}$ is an isomorphism $V_{\kappa, \kappa_{1}} \rightarrow \mathbf{R}^{2}$ ( $\epsilon$-close to the map $f_{a, b} \mapsto\left(\begin{array}{c}\pi a \\ \pi b\end{array}\right)$ ).

The two-dimensional space $V_{\kappa, \kappa_{1}}$ is a topological direct summand of $C_{0}^{j}\left(S^{1}\right)$ (the subspace $V_{\kappa, \kappa_{1}}^{\perp}$, the $\langle\cdot, \cdot\rangle$-orthogonal complement of $V_{\kappa, \kappa_{1}}$ in $C_{0}^{j}\left(S^{1}\right)$, is a closed complement). Hence $(0,0)$ is a regular value of $F$, and $\widetilde{\mathcal{K}}_{j}$ is a smooth Banach submanifold of $\widetilde{\mathcal{K}}_{j}^{1}$.

(b) (the case $j=\infty$ ) As noted in the proof of Theorem 2.8(d), $C_{0}^{\infty}\left(S^{1}\right)$ is a tame Fréchet space. Hence the affine subspace $\widetilde{\mathcal{K}}_{\infty}^{1} \subset C_{0}^{\infty}\left(S^{1}\right)$ is a tame Fréchet manifold, and so is the open subset $\widetilde{\mathcal{K}}_{\infty}^{1, \text { simp }} \subset \widetilde{\mathcal{K}}_{\infty}^{1}$, with tangent spaces all canonically isomorphic to $C_{0}^{\infty}\left(S^{1}\right)$.

Define $F: \widetilde{\mathcal{K}}_{\infty}^{1, \text { simp }} \rightarrow \mathbf{R}^{2}$ by (4.79). Then $F$ is tamely smooth and (by Lemma 4.5) $F^{-1}(0,0)=\widetilde{\mathcal{K}}_{\infty}$. By Theorem [5.5, it suffices to show that $(0,0)$ is a tame regular value of $F$.

The derivative of $F$ is given by (4.80), where now $f \in C_{0}^{\infty}\left(S^{1}\right)$. Fix $\kappa \in$ $F^{-1}(0,0)$, define $g_{1}=-\kappa \sin \circ \omega_{\kappa}, g_{2}=\kappa \cos \circ \omega_{\kappa}, V_{\kappa}=\operatorname{span}\left\{g_{1}^{\prime}, g_{2}^{\prime}\right\} \subset C_{0}^{\infty}\left(S^{1}\right)$, and, for $a, b \in \mathbf{R}, f_{a, b}=\left(a g_{1}+b g_{2}\right)^{\prime}$. From the computation in part (a), we have $\left.D F\right|_{\kappa}\left(f_{a, b}\right)=\left(\begin{array}{c}\pi a \\ \pi b\end{array}\right)$.

Thus $\left.D F\right|_{\kappa}$ maps $V_{\kappa}$ isomorphically to $\mathbf{R}^{2}$. This implies that the set $\left\{g_{1}^{\prime}, g_{2}^{\prime}\right\}$ is linearly independent, and hence the map $\iota: \mathbf{R}^{2} \rightarrow V_{\kappa},(a, b) \mapsto f_{a, b}$, is an isomorphism. Define $\hat{F}:=F \circ\left(\mathrm{id}, \iota^{-1}\right): V_{\kappa}^{\perp} \times \mathbf{R}^{2} \rightarrow \mathbf{R}^{2}$. Then $D^{(2)} \hat{F}_{\kappa}=\pi I$, and 
for $h \in C_{0}^{\infty}\left(S^{1}\right)$, 4.80) implies that

$$
\begin{aligned}
\left|D^{(2)} \hat{F}\right|_{\kappa+h}\left(\begin{array}{c}
a \\
b
\end{array}\right)-\left.D^{(2)} F\right|_{\kappa}\left(\begin{array}{c}
a \\
b
\end{array}\right) \mid & \leq\left(\left\|1-\cos \circ \omega_{h}\right\|_{0}+\left\|\sin \circ \omega_{h}\right\|\right)\left\|f_{a, b}\right\|_{0} \\
& \leq c(\kappa)\left(\|h\|_{0}^{2}+\|h\|_{0}\right)\left|\left(\begin{array}{c}
a \\
b
\end{array}\right)\right| .
\end{aligned}
$$

Hence there exists $\epsilon>0$ such that for all $\kappa^{\prime} \in B_{\epsilon}^{0}(\kappa)$, the ball in $\widetilde{\mathcal{K}}_{\infty}^{1}$ of $C^{0}$ radius $\epsilon$ centered at $\kappa,\left.D^{(2)} \hat{F}\right|_{\kappa^{\prime}}: \mathbf{R}^{2} \rightarrow \mathbf{R}^{2}$ is an isomorphism. It also follows from (4.82) (and the fact that $D^{(2)} \hat{F}_{\kappa}=\pi I$ ) that the map $B_{\epsilon}^{0}(\kappa) \rightarrow \operatorname{Hom}\left(\mathbf{R}^{2}\right)$, $\kappa^{\prime} \mapsto\left(\left.D^{(2)} \hat{F}\right|_{\kappa^{\prime}}\right)^{-1}$, is continuous, hence tame since $\operatorname{Hom}\left(\mathbf{R}^{2}\right)$ is finite-dimensional. It follows that the map $B_{\epsilon}^{0}(\kappa) \times \mathbf{R}^{2} \rightarrow \mathbf{R}^{2},\left(\kappa^{\prime}, v\right) \mapsto\left(\left.D^{(2)} \hat{F}\right|_{\kappa^{\prime}}\right)^{-1}(v)$, is continuous and tame, and therefore tamely smooth since $F$ (hence $\hat{F}$ ) is tamely smooth. Since $\iota$ is tamely smooth, the map $B_{\epsilon}^{0}(\kappa) \times \mathbf{R}^{2} \rightarrow V_{\kappa},\left(\kappa^{\prime}, v\right) \mapsto\left(\left.D^{(2)} F\right|_{\kappa^{\prime}}\right)^{-1}(v)$, is then also tamely smooth.

Thus $(0,0)$ is a tame regular value of $F$, and by Theorem $5.5, F^{-1}(0,0)=\widetilde{\mathcal{K}}^{\infty}$ is a tame Fréchet submanifold of $\widetilde{\mathcal{K}}^{1}$.

Remark 4.6. The space of functions $\kappa$ satisfying the first three constraints in Lemma 4.5 corresponds to the space of shapes of immersed smooth closed curves in the plane with rotation number 1, rather than embedded (i.e. simple) smooth closed curves. Essentially the same proof as above shows that for any $n \neq 0$, the space of immersed closed $C^{j+2}$ curves in the plane with rotation number $n$ is a smooth infinite-dimensional manifold, of Banach type if $0 \leq j<\infty$, and of tame Fréchet type if $j=\infty$.

\section{Appendix: The Nash-Moser Theorem And some Corollaries}

In this section, "smooth map" means " $C^{\infty}$ map in the Fréchet category"; see H] Definitions I.3.1.1 (p. 73), I.3.5.1 (p. 80), I.3.6.1 (p. 84), and the last line of p. 84.

To state the Nash-Moser theorem one requires definitions of "tame Fréchet space" and "tame map". The general definition of "tame Fréchet space" is intricate (see H], p. 136); however, for the applications in this paper and G1] all that matters is that $\mathbf{R}^{m}$ and all spaces of the form $C^{\infty}(M, B)$, where $M$ is a smooth compact Riemannian manifold and $B \subset \mathbf{R}^{m}$ is open, meet this definition (see [H] Theorem II.1.3.6, p. 137, and apply either Lemma II.1.3.4, p. 136, or Corollary II.1.3.9, p. 139). A map $P$ from an open subset of one space of the form $C^{\infty}\left(M, B \subset \mathbf{R}^{m}\right)$ to another space of this form is tame if for all $f_{0} \in \operatorname{dom}(P)$ there exist an integer $r$, a neighborhood $U$ of $f_{0}$, and a constant $C(n) \in \mathbf{R}$ for each integer $n \geq 0$, such that

$$
\|P(f)\|_{C^{n}} \leq C(n)\left(1+\|f\|_{C^{n+r}}\right)
$$

for all $f \in U$ and all $n \geq 0$. For a linear map between spaces of this form, tameness is equivalent to (5.1) holding globally without the " $1+$ "; this is a stronger requirement than continuity, for which the $r$ in (5.1) could depend on $n$. For a general definition of "tame map" see [H], Definition II.2.2.1, 140.

A tamely smooth map (smooth tame map in $[\mathrm{H}]$ ) is a smooth, tame map whose derivatives of all orders are tame. 
Remark 5.1 (Tame calculus). We list some important properties of tame maps and tamely smooth maps.

(1) Every continuous map from an open subset of a tame Fréchet space to a finite-dimensional vector space is tame $([\underline{\mathrm{H}}$, Example II.2.1.4, p. 141). Consequently, all smooth maps between open subsets of finite-dimensional vector spaces are tamely smooth maps.

(2) The composition of tame maps is tame $([\mathrm{H}]$, Theorem II.2.1.6, p. 142). The composition of tamely smooth maps is tamely smooth (an immediate corollary of $[\mathrm{H}$, Theorem II.2.3.3, p. 147).

(3) Let $B_{i} \subset \mathbf{R}^{m_{i}}$ be open, $i=1,2$, and $M$ a compact manifold . If $h:\left(B_{1} \subset\right.$ $\left.\mathbf{R}^{m_{1}}\right) \rightarrow\left(B_{2} \subset \mathbf{R}^{m_{2}}\right)$ is smooth, then the left-composition map

$$
\begin{aligned}
\operatorname{Comp}_{h}: C^{\infty}\left(M, B_{1}\right) & \rightarrow C^{\infty}\left(M, B_{2}\right), \\
\operatorname{Comp}_{h}(f) & =h \circ f
\end{aligned}
$$

is tamely smooth. (This is a corollary of [H], Lemma II.2.3.4, p. 147.)

(4) For $M$ any compact manifold the sum and product of tamely smooth maps from a tame Fréchet space to $C^{\infty}(M)$ is a tamely smooth map; so is the quotient if the denominator takes values in $C^{\infty}(M, \mathbf{R}-\{0\})$. (This is a corollary of the previous three facts in this list.)

(5) All tame linear maps are tamely smooth maps (because the derivative at any point is the map itself).

(6) Algebraic invertibility of each map in a tamely smooth family of linear maps, plus continuity and tameness of the family of inverses, implies that the family of inverses is tamely smooth $([\mathrm{H}]$, Theorem II.3.1.1, p. 15(1) i.e. if $E_{1}, E_{2}, E_{3}$ are tame Fréchet spaces, $U \subset E_{1}$ is open, $L_{f_{1}}: E_{2} \rightarrow E_{3}$ is an invertible linear map for each $f_{1} \in U$, such that $\left(f_{1}, f_{2}\right) \mapsto L_{f_{1}}\left(f_{2}\right)$ is a tamely smooth map, and such that $\left(f_{1}, f_{3}\right) \mapsto L_{f_{1}}^{-1}\left(f_{3}\right)$ is continuous and tame, then this latter map is a tamely smooth map.

A tame Fréchet manifold is a topological space endowed with an atlas modeled on tame Fréchet spaces and for which the overlap functions are tamely smooth maps $([\mathrm{H}, \S I I .2 .3$, p. 146). A Fréchet principal $G$-bundle is defined analogously to an ordinary principal $G$-bundle but with the underlying manifolds being Fréchet manifolds and with $G$ being a Fréchet Lie group $G([\mathrm{H}]$, Definition I.4.6.5, pp. 9899). Such a bundle is tame if all the manifolds involved in the definition are tame and all the maps involved are tamely smooth. In all of our applications, $G=S^{1}$, an ordinary Lie group, so although the Fréchet Lie group $\operatorname{Diff}^{+}\left(S^{1}\right)$ appears in part (f) of Theorem 2.8 for the sake of completeness, and in the proof of part (d) nothing for which we use Theorem 2.8 requires the reader to know what a Fréchet Lie group is.

The Nash-Moser Inverse Function Theorem can be stated as follows.

Theorem 5.2 (Tame Inverse Function Theorem). Let E, F be tame Fréchet spaces, let $U \subset E$ be open, and let $P: U \rightarrow F$ be a tamely smooth map. Suppose that for all $x \in U$, the derivative $\left.D P\right|_{x}: E \rightarrow F$ is an algebraic isomorphism and that the map $U \times F \rightarrow E$ given by $(x, v) \mapsto\left(\left.D P\right|_{x}\right)^{-1}(v)$ is tamely smooth. Then $P$ is locally invertible, and each local inverse is a tamely smooth map.

\footnotetext{
${ }^{1}$ Due to a typesetting error, the last two lines of the the cited theorem are reversed in $[\mathrm{H}]$.
} 
Just as in the Banach-space setting, there is an associated Implicit Function Theorem:

Theorem 5.3 (Tame Implicit Function Theorem). Let $E_{1}, E_{2}, F$ be tame Fréchet spaces, $U \subset E_{1} \times E_{2}$ be open, and $P: U \rightarrow F$ be a tamely smooth map. Let $D^{(2)} P$ denote the derivative of $P$ with respect to the second factor of $E_{1} \times E_{2}$. Let $\left(x_{0}, y_{0}\right) \in U$, let $z_{0}=F\left(x_{0}, y_{0}\right)$, and assume that $\left.D^{(2)} P\right|_{(x, y)}: E_{2} \rightarrow F$ is (algebraically) an isomorphism for all $(x, y) \in U$ and that the map $U \times F \rightarrow E_{2}$ given by $((x, y), v) \mapsto\left(\left.D^{(2)} P\right|_{(x, y)}\right)^{-1}(v)$ is a tamely smooth map. Then there exist open neighborhoods $V$ of $x_{0}, W$ of $y_{0}$, and a unique map $Q: V \rightarrow W$, such that for $(x, y) \in V \times W, P(x, y)=z_{0} \Longleftrightarrow y=Q(x)$. This implicitly-defined map $Q$ is tamely smooth.

Theorem 5.3 is easily derived from Theorem 5.2 using the usual trick by which the Banach-Space Implicit Function Theorem is derived from the Banach-Space Inverse Function Theorem (applying the Inverse Function Theorem to the map $(x, y) \mapsto(x, P(x, y))$; cf. [La], Theorem I.5.9).

The following definition and theorem are almost certainly not new, but the author does not know a reference.

Definition 5.4. Let $E, F$ be tame Fréchet spaces, let $V \subset E$ be open, and let $P: V \rightarrow F$ be a tamely smooth map. An element $z \in F$ is a tame regular value of $P$ if for each $x \in P^{-1}(z)$ there exists a splitting of $E$ as $E_{1} \oplus E_{2}$, with $E_{1}, E_{2}$ tame, and an open neighborhood $U$ of $x$ such that $P:\left(U \subset E_{1} \times E_{2}\right) \rightarrow F$ satisfies the hypotheses of Theorem 5.3. Analogously, if $P: M \rightarrow N$ is a tamely smooth map between tame Fréchet manifolds, we call $z \in N$ a tame regular value of $P$ if for each $x \in P^{-1}(z)$ there exist charts about $x$ and $z$, locally modeling $M$ and $N$ on tame Fréchet spaces $E$ and $F$ respectively, such that the corresponding representative of $z$ in $F$ is a tame regular value of the corresponding chart-representative of $P$.

Theorem 5.5 (Tame Regular Value Theorem). Let $M, N$ be tame Fréchet manifolds, let $P: M \rightarrow N$ be a tamely smooth map, and let $z \in N$ be a tame regular value of $P$. Then $P^{-1}(z)$ is a tame Fréchet submanifold of $M$.

The Regular Value Theorem for Banach manifolds can be proven by using either the Banach-space Inverse Function Theorem (see e.g. [M], Lemma 2.1) or the corresponding Implicit Function Theorem. Substituting Theorem 5.2 (respectively, Theorem 5.3) for the Inverse (resp., Implicit) Function Theorem in any proof of the Banach-manifold Regular Value Theorem yields a proof of Theorem 5.5. we omit the details.

\section{REFERENCES}

[CAS] I. Cohen, N. Ayache, and P. Sulger, Tracking points on deformable objects using curvature information, Computer Vision-ECCV'92, Lecture Notes in Computer Science vol. 588, G. Sandini (ed.), Springer-Verlag, Berlin, 1992, pp. 453-457.

[FB] M. Frenkel and R. Basri, Curve matching using the fast marching method, Energy Minimization Methods in Computer Vision and Pattern Recognition: Proc. 4th International Workshop, EMMCVPR 2003, A. Rangarajan et al. (eds.), Springer-Verlag, Berlin, 2003, pp. $35-51$.

[G1] D. Groisser, Certain optimal correspondences between plane curves, II: Existence, local uniqueness, regularity, and other properties, Trans. Amer. Math. Soc., this issue.

[G2] D. Groisser, Existence, local uniqueness, regularity, and other properties of certain optimal correspondences between plane curves (original version with details), preprint (2003). 
[H] R. S. Hamilton, The Inverse Function Theorem of Nash and Moser, Bull. (New Ser.) Amer. Math. Soc. 7 (1982), 65-222. MR656198(83j:58014)

[KSMJ] E. Klassen, A. Srivastava, W. Mio, and S. H. Joshi, Analysis of plane shapes using geodesic paths on shape spaces, IEEE Trans. Pattern Anal. and Mach. Intel. 26 (2004), 372-383.

[La] S. Lang, Differential and Riemannian Manifolds, Springer-Verlag, Berlin, 1995. MR 1335233 (96d:53001)

[Lo] S. Loncaric, A survey of shape analysis techniques, Pattern Recognition 31 (1998), 9831001.

[M] J.W. Milnor, Topology from the Differentiable Viewpoint, The University Press of Virginia, 1965. MR0226651 (37:2239)

[SKK] T. Sebastian, P. Klein, and B. Kimia, On aligning curves, IEEE Trans. on Pattern Analysis and Machine Intelligence 25 (2003), 116-124.

[T] H. D. Tagare, Shape-based nonrigid correspondence with application to heart motion analysis, IEEE Trans. Med. Imaging 18 (1999), 570-579.

[TOG] H. D. Tagare, D. O'Shea, and D. Groisser, Non-rigid shape comparison of plane curves in images, J. Math. Imaging and Vision 16 (2002), 57-68. MR1884465 (2002m:68120)

Department of Mathematics, University of Florida, Gainesville, Florida 32611-8105

E-mail address: groisser@math.ufl.edu 